\title{
Real Time Label-Free Monitoring of Plasmonic Polymerase Chain Reaction Products
}

A thesis submitted to McGill University in partial fulfillment of the requirements of the degree of Master of Engineering

\author{
Gideon Uchehara \\ Department of Electrical and Computer Engineering \\ McGill University, Montreal
}

August 2018

(C) Gideon Uchehara, 2018 


\section{Abstract}

The high mortality rate in developing countries stemming from poverty and diseases, and the pressure on healthcare budgets in developed countries have evoked a major concern in healthcare delivery. The need for less costly and patient-centered healthcare delivery brings point-of-care testing (PoCT) to the fore. PoCT devices help to eliminate the overheads associated with centralized bench-top laboratory instruments. Although, handheld devices such as Glucose biosensor strip exist, small handheld PoCT devices for molecular techniques such as Polymerase Chain Reaction (PCR) used to provide infectious disease testing are new and emerging. Polymerase Chain Reaction (PCR) is a biological technique used to amplify DNA. PCR makes it possible to replicate DNA and generate millions of copies from a single strand of DNA. This finds applications in the medical field to identify and detect infectious diseases. PCR is also a very important component of every laboratory involved in molecular biology experimentation. Conventional PCR equipment is expensive and require a significant amount of personnel time and space to setup and run in the laboratory. Another critical aspect of PCR systems is the need to detect amplified products, but this ability is lacking in most conventional PCR systems. Given this background, the aim of this work is to demonstrate a simple, cheap, effective and patient-centered PCR systems to mediate the shortcomings of conventional PCR machines especially as it concerns the detection of amplified PCR products.

Different methods for the detection of PCR products are described. Some of them are relatively insensitive and nonspecific while others are very sensitive and highly specific. The merits and demerits of each method are also outlined.

In this work, I have exploited the phase shift between the temperature and transmission output during PCR cycle to demonstrate a low cost and easy label-free plasmonic photodetection of PCR 
products using a simple probe laser. This method makes it possible to distinguish between negative and positive PCRs, and it can detect PCR product with starting copy number as low as 10,000 genome copies per microliter. 


\section{Abstrait}

Le taux de mortalité élevé dans les pays en développement provenant de la pauvreté et des maladies, et la pression sur les budgets des soins de santé dans les pays développés ont suscité une préoccupation majeure dans la prestation de soins de santé. La nécessité d'une prestation de soins de santé moins coûteuse et centrée sur le patient met en évidence les tests au point de service (PoCT). Les dispositifs PoCT aident à éliminer les frais généraux associés aux instruments de laboratoire de laboratoire centralisés. Bien qu'il existe des dispositifs portatifs tels que la bande de biocapteur de Glucose, de petits dispositifs PoCT portatifs pour des techniques moléculaires telles que la PCR (polymerase chain reaction ou amplification en chaîne par polymérase) utilisées pour fournir des tests de maladies infectieuses sont nouveaux et émergents.

La PCR (polymerase chain reaction ou amplification en chaîne par polymérase) est une technique biologique utilisée pour amplifier l'ADN. La PCR permet de répliquer l'ADN et de générer des millions de copies à partir d'un seul brin d'ADN. Cela trouve des applications dans le domaine médical pour identifier et détecter les maladies infectieuses. La PCR est également un élément très important de chaque laboratoire impliqué dans l'expérimentation en biologie moléculaire. Les équipements de PCR conventionnels sont coûteux et nécessitent beaucoup de temps et d'espace pour être installés et utilisés en laboratoire. Un autre aspect critique des systèmes de PCR est la nécessité de détecter les produits amplifiés, mais cette capacité fait défaut dans la plupart des systèmes de PCR conventionnels. Compte tenu de ce contexte, le but de ce travail est de démontrer un système de PCR simple, bon marché, efficace et centré sur le patient pour arbitrer les lacunes des machines PCR conventionnelles en particulier en ce qui concerne la détection des produits de PCR amplifiés. 
Différentes méthodes de détection des produits de PCR sont décrites. Certains d'entre eux sont relativement insensibles et non spécifiques alors que d'autres sont très sensibles et très spécifiques. Les mérites et les démérites de chaque méthode sont également décrits.

Dans ce travail, j'ai exploité le déphasage entre la température et la sortie de transmission au cours du cycle de PCR pour démontrer une photodétection plasmonique facile à utiliser et à faible coût de produits de PCR en utilisant un simple laser sonde. Cette méthode permet de faire la distinction entre les PCR négatives et positives, et elle peut détecter un produit de PCR avec un nombre de copies de départ aussi bas que 10,000 copies du génome par microlitre. 


\section{Acknowledgements}

I wish to express my profound gratitude to God for the gift of life and the opportunity to study in one of Canada's best institutions. A big thank you to my supervisor Professor Andrew Kirk for providing me with the needed support in the course of my masters program. His patience with me and guidance to me were key to the successful completion of my program. I would also like to thank Professor Miltiadis Paliouras for his motivation and constant support. The discussions we had gave me a better understanding of the subject of PCR. I appreciate the times he spent working with me in the lab. Many thanks to Santiago Bernal who was helpful in the CAD design of the plasmonic PCR setup. I wish to recognize the work of undergraduate students who contributed to this project: Wesley Sun and Hunter Hauswirth for their help in calibrating the IR thermometer. And Fazal Khan who supported during the initial phase of the project with the design of lock-in amplifier. My colleague, Padideh Mohammadyousef was very supportive during my thesis and provided useful input in the analysis of my data. Mr. Joseph Boka was very helpful to me in the initial stage of this project and taught me how to design lock-in amplifiers. Thanks to my friend Barizomd Elect Lebe Boogbaa for helping with the translation of the abstract from English to French. A special thank you to my wife, Dr. Chidubem E. Uchehara who supported me through the period of my master. Her encouragements, patience and support were inestimable. I would also like to acknowledge the financial support I received from the Niger Delta Development Commission (NDDC), and Society of Photo-Optical Instrumentation Engineers (SPIE) during the first year of my master's program. Finally, I wish to acknowledge the support of my parents, family members and friends who contributed in one way or the other to the success of my master's program. 


\section{Table of Contents}

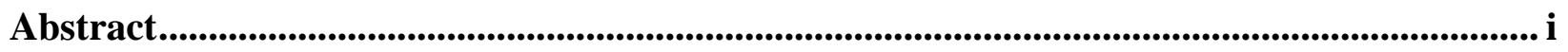

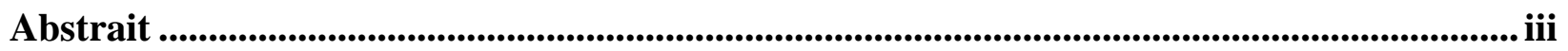

Acknowledgements ............................................................................................................................ v

Table of Contents .............................................................................................................. vi

List of Figures................................................................................................................................................. viii

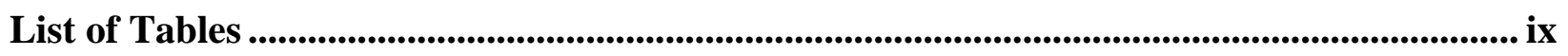

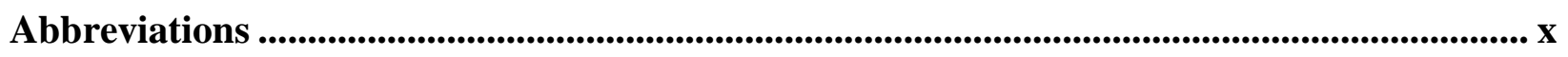

Chapter 1: Introduction ................................................................................................................................. 1

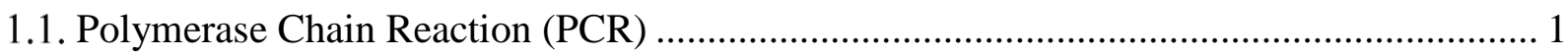

1.1.1. Traditional Methods of Detection of PCR Product ..................................................... 4

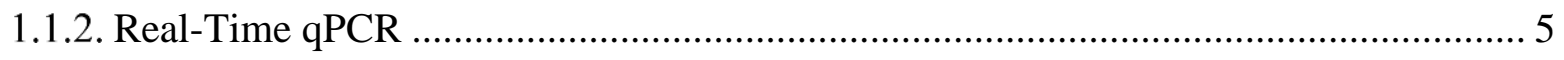

Chapter 2: Literature Review ............................................................................................... 7

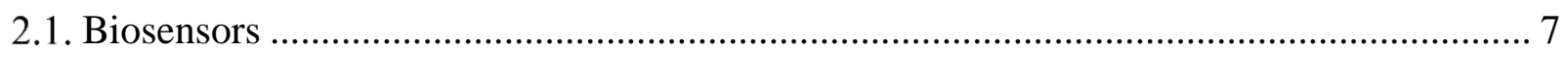

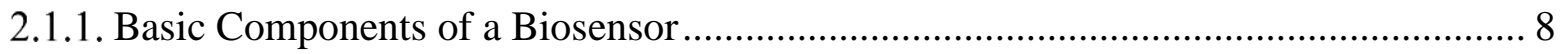

2.1.1.1. Biorecognition ........................................................................................ 8

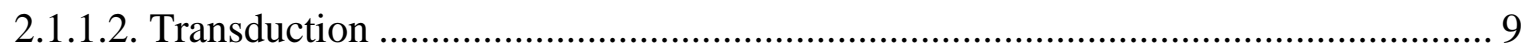

2.2. Biosensor-Based Methods for DNA Detection .......................................................... 10

2.3. Comparison of Label-Based and Label-Free Biosensors .................................................. 11

2.4. Localized Surface Plasmon Resonance (LSPR) Biosensors .............................................. 12

2.5. Factors Affecting LSPR Properties of Nanoplasmonic Sensors ....................................... 16

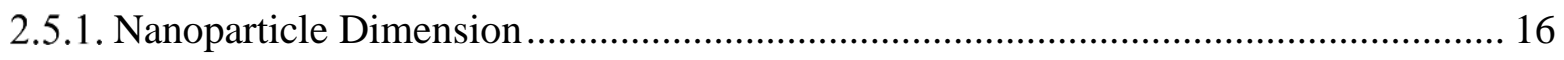

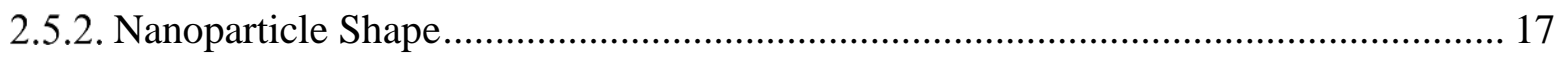

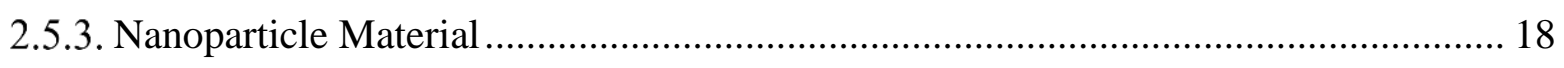

Chapter 3: Theoretical Background to Surface Plasmon Resonance ........................................ 21

3.1.1. Mie Theory for Light Scattering............................................................................ 21

3.1.2. LSPR Dependence on the Refractive Index .............................................................. 29

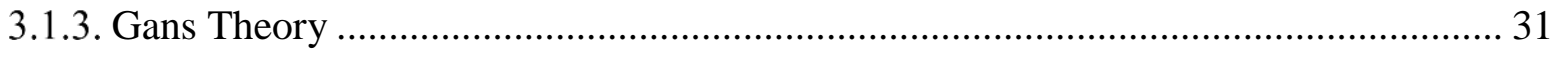

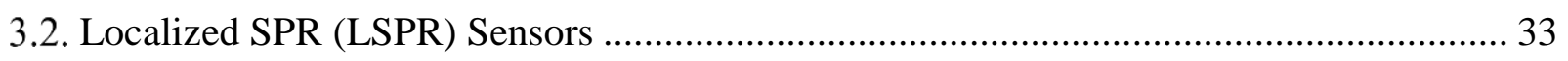

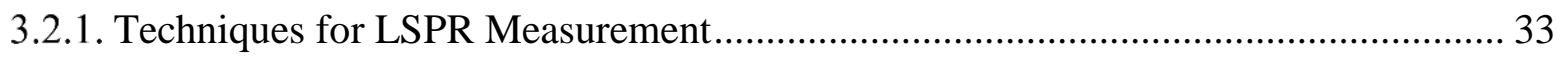

3.2.2. Refractive Index Sensing ....................................................................................... 34 


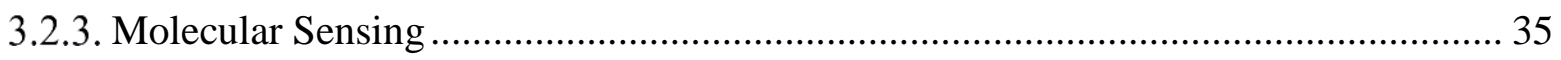

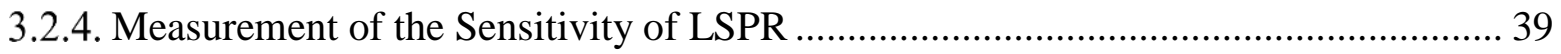

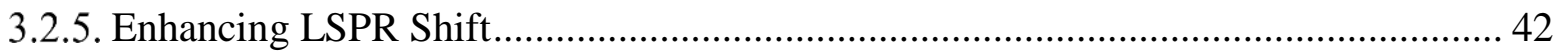

Chapter 4: Design Layout of Real-Time Plasmonic qPCR Instrument..................................... 44

4.1. Optical Thermocycling Instrument Design .................................................................. 44

4.2. LSPR Optical Sensing Instrument Design ...................................................................... 47

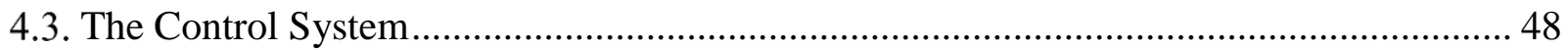

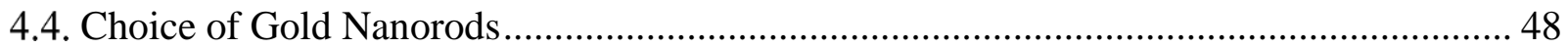

Chapter 5: Experimental Procedures/ Description of Experiments ........................................... 51

5.1. Investigating Potential Inhibition of GNRs to PCR …………………………….......... 51

5.1.1. Inhibitory concentration of bare gold nanorods (GNR) ………………………....... 51

5.1.2. Inhibitory concentration of PEGylated GNR. ………………………………......... 52

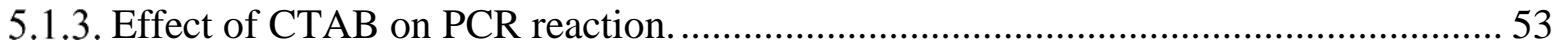

5.2. Plasmonic PCRs Using PEG-GNRs........................................................................... 53

Chapter 6: Real-Time Monitoring of DNA amplification............................................................ 58

6.1. DNA Concentration Versus Transmitted Light Intensity ............................................... 59

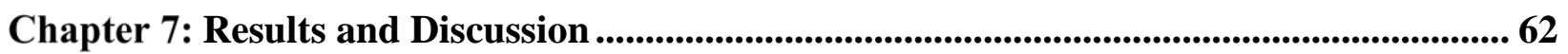

7.1. Amplitude Monitoring: Ratio of Transmission at Denaturation to Transmission at

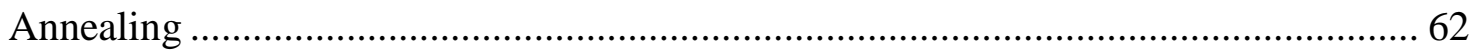

7.2. Amplitude Monitoring: Ratio of Transmission at Denaturation to Transmission at

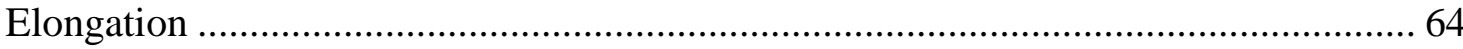

7.3. Phase shift Monitoring: Time Difference between Temperature and Transmission Curves

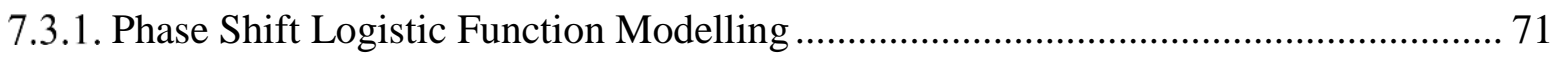

Chapter 8: Conclusion ................................................................................................................................. 75

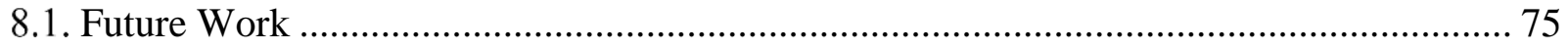

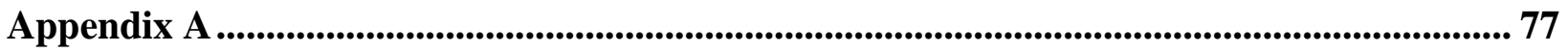

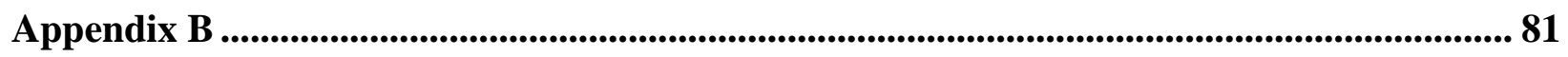

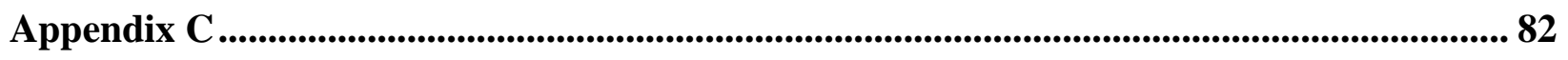

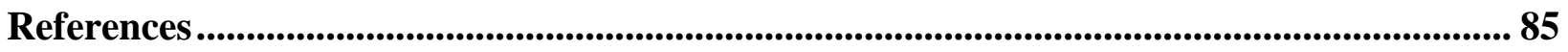




\section{List of Figures}

Figure 1.1: Overview of a Polymerase Chain Reaction (PCR) [5] .................................................... 2

Figure 2.1. Wavelength-shift LSPR sensing of the specific binding of concanavalin A to mannose-

functionalized silver nanoparticle in PBS buffer - $(6.7 \mathrm{~nm}$ red-shift) [43] .......................................... 13

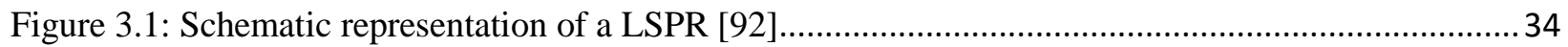

Figure 3.2: Bulk refractive index sensing versus molecular sensing by LSPR. In the case of molecular sensing by LSPR, the change to the dielectric environment of the nanoparticle is confined to the sensing

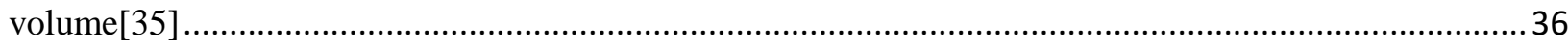

Figure 3.3: LSPR molecular detection sensitivity dependence on distance from particle surface. (a) Detecting short SAM of varying length. (b) Detecting SAM multilayers. Copyright 2004 American

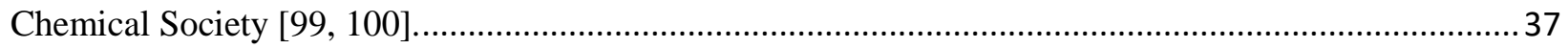

Figure 4.1: Design Layout of Real-Time Plasmonic qPCR System ..................................................4 44

Figure 5.1. Gel result for non-functionalized GNRs inhibitory test ....................................................52

Figure 5.2: Gel result for PEG-GNRs inhibitory test.................................................................... 52

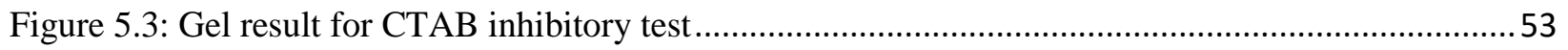

Figure 5.4: Thermocycling Temperature Curve for 30 Cycles ...........................................................55

Figure 5.5: Gel Electrophoresis Results for Plasmonic PCR ................................................................56

Figure 6.1: Plasmonic Temperature and Transmission Curves ...........................................................59

Figure 6.2: DNA Concentration Versus Transmission at $670 \mathrm{~nm}$....................................................60

Figure 7.1: Section of the Transmission curve extracted for denaturation and annealing .........................63

Figure 7.2: Plot of the ratio of Denaturation to Annealing Transmission Vs Cycle Number showing the

Slopes of the Positive and Negative PCR Curves................................................................................63

Figure 7.3: Box and Whisker Plot of Denaturation and Annealing Ratio for Negative Controls and

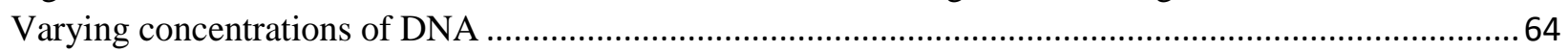

Figure 7.4: Section of the Transmission curve extracted for denaturation and Elongation .......................65

Figure 7.5: Plot of the ratio of Denaturation to Elongation Transmission Vs Cycle Number showing the

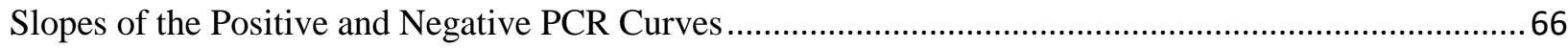

Figure 7.6: Box and Whisker Plot of Denaturation and Elongation Ratio for Negative Controls and

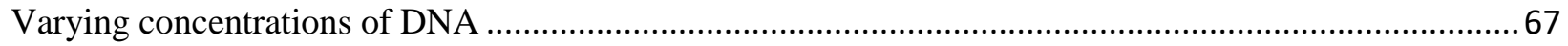

Figure 7.7. Transmission and Temperature Curves for Cycle 10 of a Negative PCR .............................68

Figure 7.8: Transmission and Temperature Curves for Cycle 30 of a Negative PCR ...............................69

Figure 7.9: Transmission and Temperature Curves for Cycle 10 of a Positive PCR................................69

Figure 7.10: Transmission and Temperature Curves for Cycle 30 of a Positive PCR .............................. 70

Figure 7.11: Time phase shift versus the cycle number for Positive and Negative PCRs ........................71

Figure 7.12: Logistic curve fits and original phase shift data plot of different PCR experiments. The vertical axis is the normalized transmission while the horizontal axis is the Cycle Number ....................73

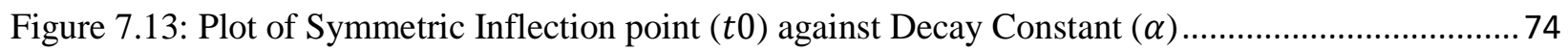




\section{List of Tables}

Table 2.1: Effect of Material Compostion on LSPR of particle of similar size and shape [35] ...............19

Table 5.1: Volume of Components of Positive and Negative PCR Reactions Mixture..............................54 


\begin{tabular}{|c|c|}
\hline \multicolumn{2}{|c|}{ Abbreviations } \\
\hline PCR & Polymerase Chain Reaction \\
\hline DNA & Deoxyribonucleic Acid \\
\hline CAD & Computer Aided Design \\
\hline FND & Ferrocenyl naphthalene diimide \\
\hline ELISA & Enzyme-linked ImmunoSorbent Assay \\
\hline LSPR & Localized surface plasmon resonance \\
\hline QCM & Quartz-crystal microbalance \\
\hline SPR & Surface plasmon resonance \\
\hline RIU & Refractive Index Unit \\
\hline GNRMP & Gold nanorod molecular probes \\
\hline SAM & Self-assembled monolayer \\
\hline YFP & Yellow fluorescent protein \\
\hline PEG & Polyethylene glycol \\
\hline RNA & Ribonucleic Acid \\
\hline AFM & Atomic Force Microscopy \\
\hline MDL & molecular detection limit \\
\hline DR & dynamic range, DR \\
\hline NSL & nanosphere lithography \\
\hline FOM & Figure of merit \\
\hline IR & Infra-red \\
\hline GNR & Gold Nanorod \\
\hline SWCNTs & Single walled carbon nanotubes \\
\hline
\end{tabular}


OD Optical Density

MWCNT Multi walled carbon nanotubes

CTAB Cetrimonium Bromide

VCSEL vertical-cavity surface-emitting laser

LAMP loop-mediated isothermal amplificatio 


\section{Chapter 1: Introduction}

\subsection{Polymerase Chain Reaction (PCR)}

The invention of polymerase chain reaction (PCR) in the year 1983 by Kary Banks Mullis revolutionized the history of biological and medical sciences. According to Mullis, Polymerase Chain reaction is an in vitro technique that results in the amplification and replication of a DNA sequence to billions fold amplitude [1-3]. This invention won him the Nobel Prize in Chemistry along with Michael Smith for his work on PCR. Mullis's concept for PCR is a combination of existing components such as the synthesis of short lengths of single-stranded DNA (oligonucleotides), complementary to opposite strands of the target DNA. This is then used to repetitively amplify the specific region of interest such that the product of one cycle of the polymerase reaction forms the template for the next cycle hence, the chain reaction [4]. PCR requires thermocycling at specific temperatures. This is done with the help of thermal cyclers which are used to heat and cool the reaction tubes within it to the different temperatures required for each stage of the PCR reaction. Some thermocycling machines have heated lid placed on the top of the reaction tubes to avoid evaporation of reaction mixture while in others, mineral oil is used. We used mineral oil on the surface of the reaction mixture to avoid evaporation. 


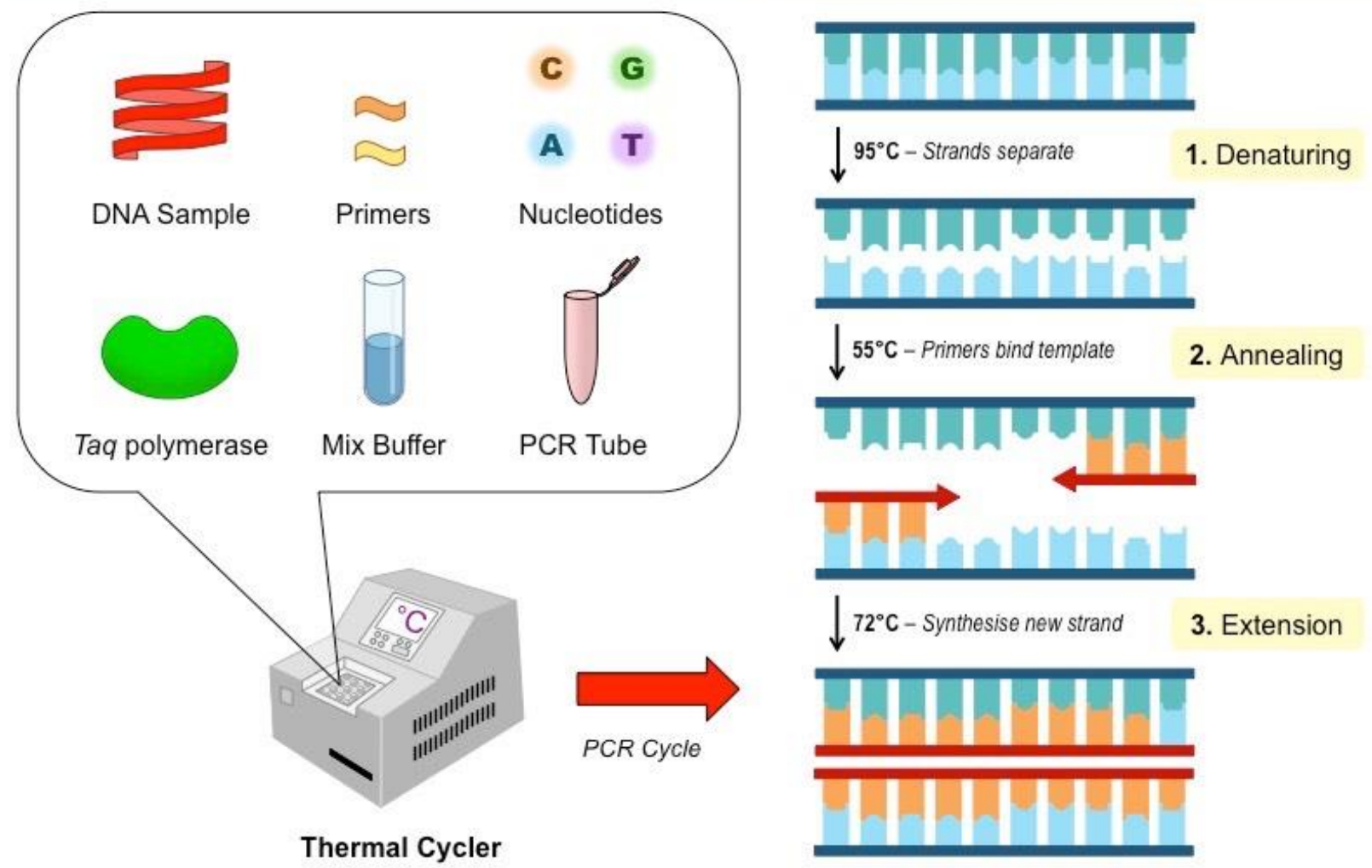

Figure 1.1: Overview of a Polymerase Chain Reaction (PCR) [5]

PCR reaction cannot proceed without important components. One of such components is the template DNA or cDNA which contains the DNA sequence to be amplified. Primers are also important components required in PCR. Usually two short, artificial DNA strands typically 1825bp in length, otherwise known as primers are used. These primers are complementary to the beginning and end of the target DNA sequence to be amplified [6]. The DNA-Polymerase is another component of the PCR which helps in the synthesis of new DNA strand by replicating the region to be amplified. The dNTPs or nucleotides are DNA building blocks from which the DNA polymerase forms new DNA. The buffer maintains the $\mathrm{pH}$ and ionic strength of the reaction solution appropriate for the activity of the DNA-Polymerase. 
PCR thermocycling process involves three steps: denaturation, annealing and elongation. These steps combined, form one cycle of the PCR process. Usually, twenty to thirty-five cycles are required in the PCR process. During the denaturation, the hydrogen bonds that binds two strands of DNA are broken apart by heating the PCR mix to 94-96 degrees Celsius. During annealing, the PCR reaction mix is cooled so that primers can anneal themselves to the single DNA strands. The type of primer used determines the annealing temperature; usually the temperature range is between 45-60 degrees Celsius. The annealing temperature is very important as any deviation from the required may result in the primers not annealing to the single DNA strands. At the elongation step, the temperature is increased to typically 72 degrees Celsius to fill in missing DNA strands. [6].

The PCR process can be divided into the exponential amplification stage, leveling off stage and the plateau stage. At the exponential stage, the efficiency of the PCR at each cycle is approximately $100 \%$ in which case the amount of the product of PCR is doubled. The Leveling off stage occurs when the DNA polymerase loses activity and when the other components of the PCR such as dNTPs and primers are depleted. At the plateau stage, no more product is formed as a result of the exhaustion of reagents and enzyme [6].

PCR finds application in forensic science and serves as an important tool in DNA profiling and fingerprint recognition. PCR enables gene testing for the presence of genetic diseases and paternity test. PCR is also useful as confirmatory diagnostic aid in infectious diseases such as HIV/AIDS, Hepatitis, cancers, tuberculosis, etc. [1, 6]. 


\subsubsection{Traditional Methods of Detection of PCR Product}

Detection of amplified PCR products is as critical to PCR as the thermocycling process. The combination of the sensitivity of the PCR reaction, along with the sensitivity and specificity of product detection makes PCR technology an important tool in the field of molecular biology and clinical microbiology. A PCR reaction may yield DNA fragments consisting of the expected amplified product along with fragments representing nonspecific amplification while another may produce a single amplified product at an amount that is detectable. It is important to have a system that is capable of detecting the desired product regardless of the number of nonspecific fragments present [7]. The merits and demerits of some basic traditional methods for detecting PCR products are reviewed.

The densitometric evaluation by electrophoresis in ethidium bromide-stained gels represents the simplest way of quantitation of PCR product. Ethidium Bromide is a well-known and widely used fluorescent dye. It intercalates between the stacked bases of double-stranded DNA causing the DNA to fluoresce when exposed to UV light at $260 \mathrm{~nm}$. PCR products are identified by the appearance of the expected DNA band when run next to the DNA markers during electrophoresis. Although this method of PCR detection is simple and inexpensive, the major disadvantage to the use of ethidium bromide is that the dye can only detect bands that contain approximately $5 \mathrm{ng}$ or more of DNA. For some experiments this may be too insensitive and therefore not appropriate. Another disadvantage of ethidium bromide is that it can lead to faulty interpretation of results since all of the DNA products (both specific and nonspecific) products are stained [7].

Specific PCR products can be identified using DNA probe that hybridizes to a specific region of the DNA located internally between the two PCR primers. The DNA probe can either be radiolabeled or labeled with nonradiative markers such as biotin or digoxigenin. Apart from 
specific detection, labelled DNA probes provides increased sensitivity over ethidium bromide staining. End-labeling of oligonucleotide with $\left[{ }^{\gamma-32} \mathrm{P}\right]$ ATP by T4 polynucleotide kinase is a common method for radiolabeling oligonucleotide probes. The procedure is fast, easy and very efficient producing labeled DNA probes with high specific activities. On the other hand, a variety of nonradioactive labels including biotin, digoxigenin, HRP and fluorescein are used for labeling DNA probes [3]. Non-radioactive methods to label DNA are often more used because of the advantages they offer over radioactive methods. Non-radioactive detection methods eliminate the need to deal with the licensing, waste disposal, and safety concerns associated with the use of radioactive material. The probes generated are more stable than are the probes labeled with ${ }^{32} \mathrm{P}$. Non-radiative detection methods require shorter exposure times to detect hybridization signal. These methods of PCR detection are laborious and time consuming $[7,8]$.

Although quantitative PCR (qPCR) using labels has been successfully used to quantify DNA amplification, the shortcomings associated with these methods of DNA quantification and the need for a higher throughput, speed, and reduction in cost of running PCR have led researchers to develop better label-free devices for monitoring real-time DNA amplification.

\subsubsection{Real-Time qPCR}

The limitations of conventional PCR led to the development of real-time qPCR. Introduced by Higuchi and co-workers in 1992, qPCR measures the amplification of DNA at each cycle of PCR using fluorescent reporter dye, such as SYBR Green. This means that detection of PCR product is done at the same time as the amplification process, thereby reducing time taken to analyze PCR products. Real-time qPCR is very sensitive and gives a real time and more precise measurement of PCR product than conventional method of PCR quantification. The intensity of the fluorescent dye corresponds to the amount of DNA produced $[9,10]$. Real-time PCR systems are designed to 
have thermal cycler that provides cycling between set temperatures for DNA amplification, an optical system which is used to excite fluorophores and capture emitted fluorescence. The intensity of the transmitted light is then correlated with the amount of DNA produce by a specialized software [1].

Real-time qPCR technology has been in use for several years now, and it is evolving into different biosensing platforms for detecting and quantifying PCR products [11]. In the next chapter, we would explore some of the different DNA biosensors that have been developed. 


\section{Chapter 2: Literature Review}

\subsection{Biosensors}

Biosensors are analytical devices used to detect and analyze biological samples. They are essential in food analysis, bioterrorism, environmental monitoring, health monitoring and diagnostics, drug screening and in many other biomedical applications. Biosensors measure changes resulting from analytes attaching onto a biorecognition element or a specific surface.

Whereas conventional analytical techniques may require a number of steps, much labor, time and expensive instruments, biosensors mediate all of these shortcomings because they are quick, simple, economical and serve as point of care diagnostic tools in remote areas where state-of-theart equipment are not available [12]. However, these biosensors are faced with certain limitations based on selectivity and specificity in detecting biomolecules. For example, DNA molecules which are sometimes present in concentration order of $\mathrm{fg} / \mathrm{ml}$ to $\mathrm{pg} / \mathrm{ml}$ with other molecules, requires a biosensor technology that is highly sensitive in order to selectively detect and distinguish it from other molecules. It is therefore important to develop biosensors that are highly sensitive, selective and multiplexed to meet the growing demand for point-of-care use.

Because of the limitations in sensitivity and selectivity, most biosensors exploit the affinity of a receptor molecule towards a specific target molecule. For example, in DNA hybridization biosensor, complementary DNA base paring forms the basis for biorecognition process. Singlestranded DNA segments are immobilized on the surface of the transducer. When DNA binds to the complementary sequence of the capture or probe DNA, electrical signal is produced. This process is called hybridization. In order to achieve high selectivity and sensitivity, hybridization conditions such as temperature, ionic strength and time must be optimized. Ferrocenyl naphthalene 
diimide (FND), Horseradish Peroxidase, alkaline phosphatase, Colloidal gold have been used as hybridization labels [12].

Sometimes, the receptor molecule is functionalized with a label, as in the case of Enzyme-linked ImmunoSorbent Assay (ELISA), in order to measure and quantify the specific antigen in the sample. This is called label-based biosensing. Even though this method of biosensing has attracted varied applications, it is faced with several challenges. For example, it is difficult to track molecular event in real-time with label-based biosensing. Also, some label-based biosensors have relatively low-throughput measurement.

As a result of the above, researchers have become interested in developing label-free biosensors that mediate the limitations of label-based biosensors, including reducing the complexity and cost, and maintaining a high throughput. Among the different label-free biosensors, localized surface plasmon resonance (LSPR) biosensor is preferred because of the high sensitivity, low cost and small environmental footprint it provides. LSPR biosensors depend on the coupling of biorecognition element with a physical transducer to convert the biological signal into an electrical or other type of signal. This work is based on LSPR biosensing.

\subsubsection{Basic Components of a Biosensor}

Basically, biosensing involves biorecognition and transduction. The following sections give a brief description of these two processes.

\subsubsection{Biorecognition}

Biorecognition involves sensing elements which may be whole cells, antibodies, enzymes or nucleic acids forming a recognition layer that is integrated with the transducer through immobilization by adsorption, cross-linking or covalent binding [12]. Biorecognition elements 
with high affinity constant for the target analyte are chosen and immobilized on the sensing surface to detect a biomolecule of interest. Biorecognition elements are chosen based on the experimental conditions. The immobilization strategy employed for the biorecognition element depends on the type of surface and the category of element. To avoid non-specific binding of biomolecules on the surface of the biorecognition element, an efficient surface chemistry is required to serve as a blocking agent to fill the empty space between the elements. [13-15]. For this thesis experiments, polyethylene glycol (PEG) is used as the blocking agent to provide to prevent the binding of DNA molecules to nanoparticles. Depending on the sensing technique, labelling may form an important part of the biorecognition step. Labels are used to enhance the signal for the detection at low concentrations. Secondary antibodies [16] and nanoparticles [17] may be used as labels. However, some sensing technique do not require labelling for detection and they are referred to as label-free. This is the method we have employed in this thesis because of the advantage that it simplifies the steps involved in biosensing process.

\subsubsection{Transduction}

Transduction is the measurement of the parameter of the change induced by the biorecognition element. Since transduction is primary based on the parameter of measurement, it may be amperometric (current measurement at constant potential), potentiometric (potential measurement at constant current), piezoelectric (measurement of changes in mass), thermal (measurement of changes in temperature) or optical (detect light changes in transmission of light) [12]. Optical biosensors a the most widely used of all the other biosensors. Optical biosensors such as waveguides [18], Mach-Zehnder interferometers [19], fluorophores [20], surface enhanced Raman Scattering [21] and microcavities [22] used light to probe samples and measure changes in refractive index. A standard optical biosensor that is commercially available as a test kit is the 
enzyme-linked immunosorbent assays (ELISA). It is popular for its sensitivity and ease of use [23, 24].

\subsection{Biosensor-Based Methods for DNA Detection}

The economic importance of DNA biosensors in the testing of genetic and infectious diseases, detection of specific DNA sequence in areas such as food, forensic examination, clinical and environmental analysis, has drawn the attention of researchers in recent years. Conventional method of genetic analysis involves the extraction of DNA from biological samples. A specific region of interest is amplified by the polymerase chain reaction (PCR) process. The product of the PCR is then detected by means of electrophoresis. Although the conventional method has proven useful in the identification of microorganisms such as Mycobacteria, Legionella sp. and viruses that are difficult to culture and identify by standard techniques, biosensor-based methods of DNA detection is gaining popularity as an alternative because of its specificity in direct detection of DNA [25].

DNA biosensors are faster, simpler and cheaper compared to traditional hybridization. Among other biorecognition elements, nucleic acids have been widely used in DNA biosensors because of their wide range of physical, chemical and biological activities [12, 25]. Unlike enzymes or antibodies, nucleic acids recognition layers can be readily synthesized and regenerated for multiple uses. The sensing elements for nucleic acid biosensors are oligonucleotides, with a known sequence of bases, or fragment of DNA or RNA [12]. The following sections highlight some DNA biosensors. 


\subsection{Comparison of Label-Based and Label-Free Biosensors}

The difficulty associated with direct detection and quantification of biological analytes has led to the development of biosensors that exploit the high affinity of a receptor molecule towards a specific biomarker. This receptor molecule is usually functionalized with a label that is easy to measure and quantify [26]. Label-based sensors utilize foreign molecules known as labels which are temporarily or chemically attached to the target molecule to detect molecular presence or activity. The labeling process could involve covalent bonding through coupling chemistry as in the case of fluorescent, chemiluminescent, and nanoparticle labeling, or isotopic in which case, special elements are incorporated into the molecules to produce a measurable difference. Electrochemically active probe labeling which involves temporary attachment of intermolecular bonding is another labeling process employed in some label-based sensors [27].

Although Label-based sensors such as Enzyme-Linked ImmunoSorbent Assay (ELISA) have been used to detect subfemtomolar concentration of target analyte [28], this method of sensing is faced with a lot of disadvantages. One major drawback to label-based biosensors is the fact that they do not permit continuous monitoring; they only provide endpoint read out thus, making it impossible to get information on the binding kinetics. Also, the use of isotope-label molecules poses great safety challenges $[29,30]$. Fluorescent label detection has relatively low-throughput measurement capability [31-33]. Label-based sensing often requires a laborious and complicated process of sample preparation steps thereby reducing the effective throughput and increasing the cost. Labelbased sensing becomes complex when it involves the identification and production of two different antibodies required for the recognition of different regions of the same target [26].

In contrast, label-free sensors utilize molecular biophysical properties such as refractive index, mass or charge for detection. Some label-free detection methods include: quartz-crystal 
microbalance $(\mathrm{QCM})$, surface plasmon resonance (SPR), localized surface plasmon resonance (LSPR), microcantilever, mass spectrometry, etc [27]. Label-free sensors provide quantitative information on continuous monitoring of binding affinity and kinetics and measures the change in the concentration of target molecules over time [26, 34]. In contrast to label-based sensors which requires two or more antibodies to the target molecule to generate and amplify the target signal, the measured signal in a label-free sensor is usually due to the target molecule alone [35].

\subsection{Localized Surface Plasmon Resonance (LSPR) Biosensors}

Localized surface plasmon resonance (LSPR) biosensors are label-free biosensors that exploits the sensitivity property of plasmon frequency to changes in the local refractive index of the medium at the nanoparticle surface. LSPR results from the interaction of light with particles whose sizes are smaller than the incident wavelength. LSPR biosensors are more sensitive, robust and offer simpler detection compared to other label-free biosensing techniques. As such LSPR biosensors have gained preference over other biosensors [36].

LSPR sensors do not require complex instrumentation or scanning optics as those used with SPR sensors. LSPR mode can easily be excited by illuminating a nanostructure with a light source, regardless of the angle of incidence. Different platforms exist that utilizes LSPR; however, the common LSPR sensing modes is the wavelength-shift LSPR sensing mode. In wavelength-shift LSPR sensing, the change in maximum (or minimum) of the LSPR extinction curve is correlated with changes in the local refractive index of the surrounding medium caused by analyte adsorption.

LSPR sensing systems have been developed to demonstrate the sensitivity of nanoparticles to their local environment. For example, the binding of streptavidin or antibiotin to biotin-functionalized nanoparticle arrays, and the binding of concanavalin A to mannose-functionalized nanoparticles 
resulted in a measurable shift in the maximum wavelength of the nanoparticle as shown in figure 2.1 [37-39]. The sensitivity of streptavidin ranges from picomolar to micromolar concentrations. This has the potential of increasing to femtomolar sensitivity because it has been discovered that in solution, biotin-streptavidin binding constant is of the order of $10^{14} M^{-1}[35,40]$. Successful application of Streptavidin detection in serum and dillute blood has been recorded [41, 42].

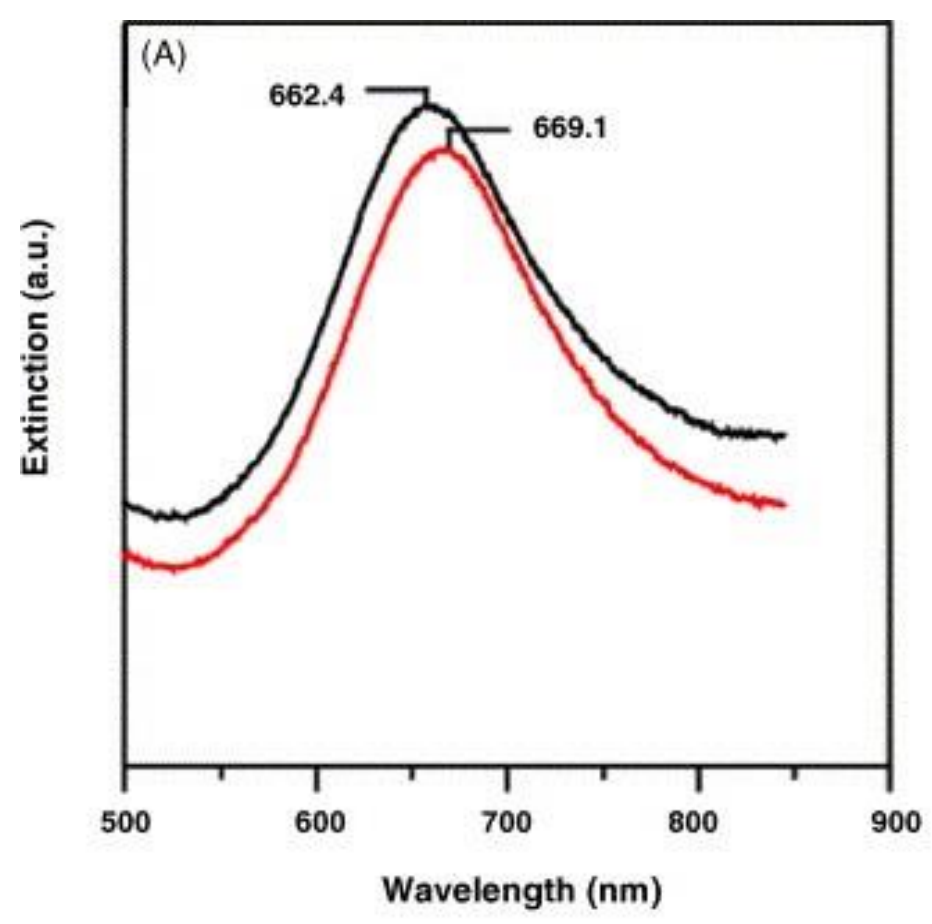

Figure 2.1. Wavelength-shift LSPR sensing of the specific binding of concanavalin A to mannose-functionalized silver nanoparticle in PBS buffer - (6.7nm red-shift) [43]

LSPR sensors to analyze antigen-antibody binding have be demonstrated. This LSPR nanosensor has been successfully used in the detection of Alzheimer disease.[44] The sensitivity of these sensors which depends on the binding constant and signal-to-noise ratio, have be found to be in the range $10^{9}-10^{10} M^{-1}$ [45-47]. It is possible to monitor the real-time analysis of antigen- 
antibody binding kinetics with gold nanorod LSPR assay. This helps to determine the specificity within a single experiment and over the same nanoparticles [35].

Bright Field Microscopy which provides a direct measurement of extinction (absorption and scattering) of nanostructures utilizes LSPR. Dark field microscopy is an improved version of the bright field microscopy. It is widely used in protein association [48]. The advantage dark field microscopy has over the former is that it helps to reduce the effect of background noise and it can measure the scattering from a single nanoparticle [49]. Advances in dark field microscopy for LSPR imaging have been reported [50].

LSPR spectroscopy based on reflection measurement also exits. This type of spectroscopy is used to measure the reflection from nanoparticles when the scattering cross section dominates over the absorption cross section, in which case the scattering of the nanoparticle contributes more to the total extinction efficiency of the nanoparticle. The reflection measurement can be performed by reflecting probe or integration sphere [49]. Another simple but important LSPR spectroscopy is that based on direct measurement of transmitted light. This is the method used to obtain the results for this thesis. This method involves the direct excitation of nanostructure (nanoparticle solution in this case) with a light beam. The transmitted light is then measured by a photodetector.

Metal nanostructures have shown remarkable optical properties that are employed in biosensing application. When light is incident on a periodic metal nanostructure of subwavelength aperture, the transmitted light is usually higher than that expected by standard aperture theory. This phenomenon has led to the development of a hybrid biosensing platform in which suspended nanohole arrays were used as plasmonic sensors in which analytes are transported through nanoholes thereby maximizing the ligand-analyte binding event [52-58]. This sensor has a sensitivity of about $630 \mathrm{~nm} / \mathrm{RIU}$ [49]. Other metallic nanostructures such as gold nanoshells that 
support a tunable LSPR mode have been developed with better sensitivity to bulk refractive index [59]. There are also metallic nanostructure sensors based on wavelength division multiplexing that mediate the challenges of spectral multiplexing associated with propagating SPR [60]. Gold nanorod molecular probes (GNRMP) sensors provide more sensing channels by tuning the aspect ratio of the nanorods functionalized to different target analytes. A major disadvantage of this sensor is the aggregation and cross talk resulting from the instability of the sensor in the solution $[49,61]$. Surface-enhanced spectroscopic processes rely on LSPR for their electromagnetic-field enhancement.

LSPR sensors have been used to probe nucleic acid hybridization. For example, LSPR-based detection of the BIGH3 gene mutation, which are associated with corneal dystrophy, using a multispot gold-caped nanoparticle array have been demonstrated with a detection limit of $1 \mathrm{pM}$ [62]. LSPR sensors based on gold nanospheres functionalized with glucose-bearing polymer chains were used to study the binding of ConA to glucose. The limit of detection was found to be $1.9 \mathrm{nM}$ and the kinetics was measured to be over 6 orders of magnitude of the target concentration [63]. LSPR sensing based on biomolecular interaction of small drug molecule with cytochrome has been reported. In this type of sensors, when the LSPR peak wavelength matches the optical absorbance of the biomolecule, the nanoparticle experiences a greater LSPR wavelength shift. Because cytochrome has absorption bands that overlap the LSPR of silver nanotriangles, when the nanoparticles are functionalized with cytochrome P450, a resultant blue- or red-shift in the nanoparticle extinction is observed. This shift is usually $3-5$ times larger than the shift induced by the refractive index change produced by the same small molecules $[64,65]$. This same principle of LSPR sensing has been applied to measure the response to three different drugs: ketoconazole, 
testosterone and erythromycin. These drugs showed a large red-shift, a large blue-shift and a negligible shift respectively $[35,66]$.

LSPR sensing based on silver nanocubes functionalized with an alkanethiol SAM have been used to monitor in real-time, the binding of neutravidin to membrane-bound biotin and the binding of functionalized lipid molecules to yellow fluorescent protein (YFP). The results show that the unbinding rate of YFP was comparable to that measured by fluorescence microscopy [67]. Gold nanospheres deposited on an unclad section of an optical fiber and functionalized with acetylcholinesterase (AChE) was used as LSPR biosensor to detect paraoxon, an organophosphorus pesticide, with detection limit of $0.234 \mathrm{ppb}$ [68]. Another researcher has demonstrated the use of gold nanorods coated with PEG-thiol (polyethylene glycol) as LSPR sensor to detect dithiol molecules including gluatathione and cysteine [69]. In another development, LSPR biosensor in which antifibrinogen antibodies were linked to gold-capped nanoparticle substrate via RNA aptamer was used to detect fibrinogen with a detection limit of $0.1 \mathrm{ng} / \mathrm{ml}[69]$.

\subsection{Factors Affecting LSPR Properties of Nanoplasmonic Sensors}

\subsubsection{Nanoparticle Dimension}

The size and aspect ratio of nanoparticles affects its optical properties such as the resonance wavelength, the extinction cross-section, and the ratio of scattering to absorption. As a result, it is important to consider the effect of size and dimension in the selection of nanoparticles for lightscattering and absorption-based application in biomedicine. Nanoparticles are used as nanoplasmonic sensors based on the optical properties they are designed to meet. For example, gold nanorods with a smaller effective radius and a higher aspect ratio is best for photoabsorbing 
application. On the other hand, nanorods with a larger effective radius and high aspect ratio finds application in scattering contrast for imaging [70].

According to Mie theory, the magnitude of the absorption and scattering cross-section varies proportionately as $R^{3}$ and $R^{6}$ respectively, for spherical particles of radius $R$ much less than the wavelength of light $(2 \pi R \ll \pi)$. As a result, absorption dominates LSPR extinction for smallest nanoparticles. However, increasing the size of the particle results in the dominance of the scattering cross-section in the LSPR extinction. This transition occurs at around 80nm in particle diameter for gold nanospheres [70]. In his study of dark field microscopy of gold colloid, Gustave Mie discovered that the LSPR wavelength depended on the size of the particle. The LSPR Wavelength of gold nanospheres can be tuned over $60 \mathrm{~nm}$ by varying the particle size between $10 \mathrm{~nm}$ to $100 \mathrm{~nm}$ $[35,71]$. The plasmon resonance bandwidth has been found to increase for small particles due to interband transition. It also increases for larger particles because of higher-order plasmon modes [71].

Aspect ratio is the ratio of length to width of a particle. The aspect ratio of nanoparticles affects their refractive index sensitivity. For example, increasing the aspect ratio of gold bipyramids from 1.5 to 4.7 was demonstrated to increase the sensitivity from $150 \mathrm{~nm} / \mathrm{RIU}$ to $540 \mathrm{~nm} / \mathrm{RIU}$ [72]. Also, increasing the aspect ratio of gold nanorods of radius $10 \mathrm{~nm}$, from 1.0 to 3.4 was found to increase the sensitivity from $157 \mathrm{~nm} / \mathrm{RIU}$ to $497 \mathrm{~nm} / \mathrm{RIU}$ [70].

\subsubsection{Nanoparticle Shape}

Apart from the aspect ratio, it has been demonstrated that the shape of nanoparticles affects their sensitivity. This has led to the development of different nanoparticles shapes used as biosensors. These nanoparticles can be spherical, triangular or cubic in shape. Nanoparticles with sharp tips 
produce greater refractive index sensitivities [35]. For example, it was demonstrated that the refractive index sensitivity of nanotriangles $(350 \mathrm{~nm} / \mathrm{RIU})$ are greater than nanospheres (160nm/RIU) [73]. In another demonstration, it was shown that the sensitivity of gold nanosphere (60nm/RIU) was much less than that of gold nanoshell (409nm/RIU) [74].

Other nanoparticle shapes of a great deal of interest, that have been developed through lithography include gold nanostars, gold nanocrescent and gold bipyramids, amongst others. The sharp tips in these nanoparticles produces a redshift in the LSPR wavelength. Since for a given material composition, the refractive index sensitivity varies linearly with the LSPR wavelength, the shape of the nanoparticle plays a key role in determining the optical properties of the nanoparticle [75]. Although plasmon resonance wavelength has a greater effect on the sensitivity of the nanoparticles, the intrinsic shape of the nanoparticles also contributes to the sensitivity of the nanoparticles. These sharp features of nanoparticles can be exploited for molecular detection at the microscopic level because they create a localized sensing/mode volume of highly enhanced electric field intensity. Hence, the shape of nanoparticles are important for biosensing application [35].

\subsubsection{Nanoparticle Material}

Nanoparticles material composition play an important part in their optical properties. In fact, it has been shown that nanoparticle material has much greater effect on the optical properties than the particle size [76]. Although other plasmonic nanoparticles whose sensing applications are yet to be explored exist, gold and silver nanoparticles have found a wide application in LSPR sensing. Gold is preferred to silver because of its chemical stability and resistance to oxidation. However, silver nanoparticles have sharper LSPRs and are more sensitive to changes in the local index of refraction than gold nanoparticles. For example, silver nanospheres of 50-60nm in diameter, have 
a greater refractive index sensitivity of $160 \mathrm{~nm} / \mathrm{RIU}$ at approximately $435 \mathrm{~nm}$ plasmon resonance, compared to gold nanospheres of the same size which have refractive index sensitivity of 60nm/RIU for a plasmon resonance at approximately 530nm [73, 74]. Also, silver nancubes of 30$50 \mathrm{~nm}$ in size, have higher refractive index sensitivity of $146 \mathrm{~nm} / \mathrm{RIU}$ for a plasmon resonance at $510 \mathrm{~nm}$, compared with gold nancubes of same size whose refractive index sensitivity is $83 \mathrm{~nm} / \mathrm{RIU}$ for a plasmon resonance at $538 \mathrm{~nm}[72,77]$. Table 2.1 presents a summary of the effect of material composition on the LSPR of nanoparticles of comparable size and shape.

Particle type $\quad$ Size $(\mathrm{nm}) \quad$ LSPR peak (nm) Sensitivity (nm/RIU)

$\begin{array}{lrrr}\text { Gold nanospheres } & 50 & 530 & 60 \\ \text { Silver } & 60 & 435 & 160\end{array}$

nanospheres

$\begin{array}{llll}\text { Gold nanocube } & 44 & 538 & 83\end{array}$

$\begin{array}{llll}\text { Silver nanocube } & 30 & 510 & 16\end{array}$

Table 2.1: Effect of Material Compostion on LSPR of particle of similar size and shape [35]

The dielectric functions of gold and silver play a significant role in their different refractive index sensitivities. Since the LSPR $\lambda_{\max }$ occurs at the wavelength where $\epsilon_{i}(\lambda)$ is small and $\epsilon_{r}(\lambda)=$ $-2 \epsilon_{m}$, the real dielectric function of silver increases with wavelength more than that of gold over the visible light region. This is especially so at wavelengths between $400-600 \mathrm{~nm}$ where the plasmon resonance lies for most values of the dielectric constant of the surrounding medium. However, at higher wavelengths where the slope of the real parts of the dielectric function of the 
two metals are similar, this difference is less visible [35]. Silver nanoparticles have higher scattering efficiency, narrower plasmon linewidths and less plasmon damping compared to gold nanoparticles because, the imaginary part of the dielectric function of silver is less than that of gold in the visible region [70]. This offers silver a higher preference to gold in sensing applications because, it provides a more accurate, sharper and stronger LSPR wavelength peak [35]. 


\section{Chapter 3: Theoretical Background to Surface Plasmon Resonance}

\subsubsection{Mie Theory for Light Scattering}

This theory, discovered by Gustav Mie in 1908 when he solved Maxwell's equation for the scattering and absorption of light by a homogenous sphere, is used to model light scattering by a spherical, isotropic and non-magnetic particle in a non-absorbing isotropic medium. Mie theory can also be extended to compute other spherically symmetric geometries such as dielectric particles with metallic coatings.

The Maxwell equation assuming no sources is given by: [78-80]

$$
\nabla \times E(r, t)=-\frac{\partial H(r, t)}{\partial t}
$$

$$
\nabla \times H(r, t)=\varepsilon(r) \frac{\partial E(r, t)}{\partial t}
$$




$$
\text { D. } E(r, t)=0
$$

$\nabla \cdot \mathrm{H}(r, t)=0$

For a time-varying electromagnetic-field $(E, H)$ in an isotropic medium, the following Helmholtz wave equations hold:

$$
\nabla^{2} E+k^{2} E=0
$$

$$
\nabla^{2} H+k^{2} H=0
$$

Where

$$
k^{2}(r)=\frac{\omega^{2} \varepsilon(r) \mu(r)}{c^{2}}
$$


Because of the symmetry of a sphere, the scattered fields can be expressed in terms of vector spherical harmonics $M_{n}$ and $N_{n}$ in place of $E$ and $H$ respectively. Where the subscript $n$ indicates that different vector spherical harmonics are used to describe dipolar $((n=1)$, quadrupolar $((n=$ 2), etc. contributions to the scattered field. The following equations shows the relationship between the scattered fields to the vector spherical harmonics.

$$
\begin{gathered}
E_{s c}=\sum_{n=1}^{\infty} E_{n}\left(i a_{n} N_{e 1 n}-b_{n} M_{01 n}\right) \\
H_{s c}=\frac{k}{\omega \mu} \sum_{n=1}^{\infty} E_{n}\left(i b_{n} N_{01 n}-a_{n} M_{e 1 n}\right)
\end{gathered}
$$

Where

$$
E_{n}=l^{n} E_{0} \frac{(2 n+1)}{n(n+1)}
$$

where

$E_{0}$ is the incident field, the subscripts of $M$ and $N,{ }^{\prime} 0^{\prime}$ and $e$ represent the odd and even branches of the azimuthal solution of the vector form of the Helmholtz wave-equation; 1 denotes that $m=$ 1 terms of the Legendre and Bessel series. The Mie coefficients $a_{n}$ and $b_{n}$, determine the relative 
amplitudes of the vector spherical harmonics when excited with a particular wavelength of light. They are given as:

$$
\begin{gathered}
a_{n}=\frac{\bar{n}^{2} j_{n}(\bar{n} x)\left[x j_{n}(x)\right]^{\prime}-\bar{\mu} j_{n}(x)\left[\bar{n} x j_{n}(\bar{n} x)\right]^{\prime}}{\bar{n}^{2} j_{n}(\bar{n} x)\left[x h_{n}^{(1)}(x)\right]^{\prime}-\bar{\mu} h_{n}^{(1)}(x)\left[\bar{n} x j_{n}(\bar{n} x)\right]^{\prime}} \\
b_{n}=\frac{\bar{\mu} j_{n}(\bar{n} x)\left[x j_{n}(x)\right]^{\prime}-j_{n}(x)\left[\bar{n} x j_{n}(\bar{n} x)\right]^{\prime}}{\bar{\mu} j_{n}(\bar{n} x)\left[x h_{n}^{(1)}(x)\right]^{\prime}-h_{n}^{(1)}(x)\left[\bar{n} x j_{n}(\bar{n} x)\right]^{\prime}}
\end{gathered}
$$

Where $\bar{n}=n_{1} / n_{2}$ is the relative refractive index ( $n_{1}$ and $n_{2}$ are the complex refractive index of the particle and refractive index of the surrounding medium respectively). $\bar{\mu}=\mu_{1} / \mu_{2}$, where $\mu_{1}$ and $\mu_{2}$ are the complex relative magnetic permeability of the particle and the complex relative magnetic permeability of the surrounding medium respectively. $x=2 \pi n_{2} a / \lambda$ is the size parameter. $a$ is the radius of the sphere and $\lambda$ is the wavelength in vacuum, $j_{n}$ and $h_{n}$ are the spherical Bessel functions and spherical Hankel functions of order $n$, respectively. The primed terms in equations 3.11 and 3.12 indicate differentiation of the functions with respect to their argument.

The magnitudes of the scattering cross-section, $\sigma_{s c a}$ is the integral of the modulus of the scattered electric-field squared across a spherical surface in the far-field, which is subsequently normalized by the incident irradiance $\left|E_{i}\right|^{2}$. 


$$
\sigma_{s c a}=\frac{k}{\left|E_{i}\right|^{2}} \int_{d s}\left|E_{\text {scattered }}\left(r^{\prime}\right) \cdot \hat{r}\right|^{2} d s
$$

The scattered fields produced by a plane incident on a homogeneous conducting spherical surface results in the following total scattering $\left(\sigma_{s c a}\right)$, extinction $\left(\sigma_{e x t}\right)$, and absorption $\left(\sigma_{a b s}\right)$ crosssections: [81]

$$
\begin{gathered}
\sigma_{s c a}=\frac{2 \pi}{|k|^{2}} \sum_{n=1}^{\infty}(2 \mathrm{n}+1)\left(\left|a_{n}\right|^{2}+\left|b_{n}\right|^{2}\right) \\
\sigma_{\text {ext }}=\frac{2 \pi}{|k|^{2}} \sum_{n=1}^{\infty}(2 \mathrm{n}+1)\left[\operatorname{Re}\left(a_{n}+b_{n}\right)\right]
\end{gathered}
$$

$$
\sigma_{a b s}=\sigma_{e x t}-\sigma_{s c a}
$$

where $k$ is the incoming wavevector and $n$ are integers representing the dipole, quadrupole, and higher multipoles of the scattering. 
The scattering efficiency $Q_{s c a}$, is the scattering cross-section normalized by the geometrical crosssectional area of the scatterer. Both terms are often used to evaluate the electromagnetic scattering response of metallic nanoparticles and they can be expressed by the following equations [80].

$$
Q_{s c a}=\frac{\sigma_{s c a}}{\pi a^{2}}
$$

Assuming a nanoparticle whose size is very small compared to the size of the wavelength of light, $x \ll 1$, then the Mie coefficients $a_{n}$ and $b_{n}$, can be approximated by power series. Applying Bohren and Huffman[81], and keeping only terms to order of $x^{3}$, then $a_{n}$ and $b_{n}$ can be simplified to:

$$
a_{1} \approx-\frac{i 2 x^{3}}{3} \frac{m^{2}-1}{m^{2}+2}
$$

$$
b_{1} \approx 0
$$


where $m=\tilde{n} / n_{m}$ and $\tilde{n}=n_{R}+i n_{I}$. Here, $\tilde{n}$ is the complex refractive index of the metal, and $n_{m}$ is the real refractive index of the surrounding medium.

when keeping terms to $x^{3}$, the higher order terms of $a_{n}$ and $b_{n}$ are zero. By substituting $m=$ $\left(n_{R}+i n_{I}\right) / n_{m}$ into equation 3.18, we can find the real part of the $a_{1}$, since it is required in equation 3.15 to calculate the extinction cross-section.

$$
a_{1}=-i \frac{2 x^{3}}{3} \frac{n_{R}^{2}-n_{1}^{2}+i 2 n_{R} n_{I}-n_{m}^{2}}{n_{R}^{2}-n_{1}^{2}+i 2 n_{R} n_{I}+2 n_{m}^{2}}
$$

The complex dielectric function of the metal, $\tilde{\varepsilon}=\varepsilon_{1}+i \varepsilon_{2}$ is related to the refractive index as follows:

$$
\varepsilon_{1}=n_{R}^{2}-n_{I}^{2}
$$

$$
\varepsilon_{2}=2 n_{R} n_{I}
$$

Substituting equations 3.21 and 3.22 with the dielectric function of the medium, $\varepsilon_{m}=n_{m}{ }^{2}$ into equation 3.20 gives: 


$$
a_{1}=\frac{2 x^{3}}{3} \frac{-i \varepsilon_{1}{ }^{2}-i \varepsilon_{1} \varepsilon_{m}+3 \varepsilon_{2} \varepsilon_{m}-i \varepsilon_{2}{ }^{2}+i 2 \varepsilon_{m}{ }^{2}}{\left(\varepsilon_{1}+2 \varepsilon_{m}\right)^{2}+\left(\varepsilon_{2}\right)^{2}}
$$

By substituting equation 3.23 into 3.15 gives the renowned expression for nanoparticle plasmon resonances:

$$
\sigma_{e x t}=\frac{18 \pi \varepsilon_{m}{ }^{3 / 2} V}{\lambda} \frac{\varepsilon_{2}(\lambda)}{\left[\varepsilon_{1}(\lambda)+2 \varepsilon_{m}\right]^{2}+\left[\varepsilon_{2}(\lambda)\right]^{2}}
$$

The scattering cross-section is given as:

$$
\sigma_{e x t}=\frac{32 \pi^{4} \varepsilon_{m}^{2} V^{2}}{\lambda^{4}} \frac{\left(\varepsilon_{1}-\varepsilon_{m}\right)^{2}+\left(\varepsilon_{2}\right)^{2}}{\left(\varepsilon_{1}+2 \varepsilon_{m}\right)^{2}+\left(\varepsilon_{2}\right)^{2}}
$$

where $V$ is the volume of the nanoparticle. It is important to note that the above nanoparticle approximations for scattering and extinction cross-sections apply strictly to very small particles $(<10 \mathrm{~nm}$ diameter). However, they are accurate in the prediction of the dielectric sensitivity of large particles [82]. 
At the condition where $\varepsilon_{1}=-2 \varepsilon_{m}$, the extinction cross-section is maximum since the denominator is minimized. This is the reason why the LSPR extinction peak depends on the dielectric of the surrounding environment. For example, the LSPR peak of gold nanoparticle in water $\left(\varepsilon_{m} \approx 1.7\right)$ occurs at about $520 \mathrm{~nm}$ since at this wavelength $\varepsilon_{1}=-2 \varepsilon_{m}$. Experimental data of the absorption spectrum confirms a strong peak at that wavelength for gold colloid. While the slope of the real part of the dielectric function in the observed wavelength range is responsible for the sensitivity of the nanoparticle to $\varepsilon_{m}$, the imaginary part of the dielectric function is responsible for the damping or resonance peak broadening of the plasmon resonance. It is important to note that the surrounding dielectric constant also affects the LSPR wavelength. For example, the LSPR for silver at a given surrounding dielectric constant will be blue-shifted compared to gold in the same dielectric medium. Although silver has a lower loss due to damping effect, gold is usually preferred for most experimental applications because of its chemical stability since, it is less prone to oxidation.

\subsubsection{LSPR Dependence on the Refractive Index}

The Drude model of the electronic structure of metals which describes the collisions between freely moving electrons and the lattice of heavy, stationary ionic cores, provides a good approximation for of the conduction of noble metals and can be used to model the LSPR peak wavelength dependence on the refractive index of the surrounding medium. From the analytical, frequencydependent form for $\varepsilon_{1}$, the following equation holds: [35, 83] 


$$
\varepsilon_{1}=1-\frac{\omega_{p}^{2}}{\gamma^{2}+\omega^{2}}
$$

where $\omega_{p}$, and $\gamma$ are the plasmon frequency and damping frequency of the bulk metal respectively. $\gamma \ll \omega_{p}$ in the visible and ultraviolet regions of the spectrum hence, the Drude equation can be further simplified to:

$$
\varepsilon_{1}=1-\frac{\omega_{p}^{2}}{\omega^{2}}
$$

At resonance, $\varepsilon_{1}=-2 \varepsilon_{m}$ then,

$$
\omega_{\max }=\frac{\omega_{p}}{\sqrt{2 \varepsilon_{m}+1}}
$$

where $\omega_{\text {max }}$ is the LSPR peak frequency. Using the relation, $\lambda=2 \pi c / \omega$, and $\varepsilon_{m}=n^{2}$, we can convert the above equation from frequency to wavelength and hence, obtain the relationship between the peak wavelength and the refractive index of the medium as follows: 


$$
\lambda_{\max }=\lambda_{p} \sqrt{2 n_{m}^{2}+1}
$$

Where $\lambda_{\max }$ and $\lambda_{p}$ are the LSPR peak wavelength and the plasmon wavelength of the bulk metal respectively. The above equation shows that the LSPR peak wavelength varies linearly as the refractive index of the medium at optical wavelengths. This linearity is pronounced over a small range of refractive index for LSPR peak frequencies or wavelengths.

\subsubsection{Gans Theory}

Gans theory is an extension of Mie theory to spheroidal particles much smaller than the excitation wavelength. He found that the absorption cross-section is dependent on the aspect ratio of the particles. According to this theory, the absorption cross-section for a prolate spheroid is given as: $[35,84]$

$$
\sigma_{a b s}=\frac{\omega}{3 c} \varepsilon_{m}^{3 / 2} V \sum_{j} \frac{\left(1 / P_{j}^{2}\right) \varepsilon_{2}}{\left\{\varepsilon_{1}+\left[\frac{1-P_{j}}{P_{j}}\right] \varepsilon_{m}\right\}^{2}+\varepsilon_{2}^{2}}
$$

The sum over $j$ accounts for the depolarization factors $\left(P_{x}, P_{y}\right.$ and $\left.P_{z}\right)$ for the dimensions of the particle. Where $x>y=z$ for a prolate spheroid. The values of $\varepsilon_{1}$ and $\varepsilon_{2}$ and hence, the LSPR peak frequencies are altered anisotropically by the depolarization factors which are given as: [35] 


$$
P_{x}=\frac{1-e^{2}}{e^{2}}\left[\frac{1}{2 e} \ln \left(\frac{1+e}{1-e}\right)-1\right]
$$

$$
P_{y}=P_{z}=\frac{1-P_{x}}{2}
$$

where $e$ is a factor which includes the particle aspect ratio $R$, and it is expressed as: [85-87]

$$
e=\left[1-\left(\frac{y}{x}\right)^{2}\right]^{1 / 2}=\left(1-\frac{1}{R^{2}}\right)^{2}
$$

From equation 3.30, the corresponding extinction spectrum gives two peaks: the transverse plasmon mode peak and the longitudinal plasmon mode peak. While the former results from the contributions of $x$ and $y$ to the sum, the later is from the $z$ contribution. It is obvious from equation 3.30 that increasing the aspect ratio of the nanoparticle results in a corresponding increase in the sensitivity of the nanoparticle to the refractive index of the surrounding medium and hence, redshift in the plasmon peak wavelength. Apart from spheres and spheroids, for other nanoparticles, the shapes determine the LSPR spectrum [88]. 


\subsection{Localized SPR (LSPR) Sensors}

LSPR sensors are non-propagating surface plasmon sensors. They are excited on nanostructured metal surfaces and the LSP resonance can be tuned by changing the size, shape and composition of the nanoparticle [89]. Unlike the propagating SPR sensors, LSPR sensors do not require prism or grating coupling to excite the conduction electrons. Light can be absorbed or scattered by the metal nanoparticle if the wavelength of the incident light matches the resonance wavelength of the metal nanoparticle.

\subsubsection{Techniques for LSPR Measurement}

Nanoparticles convert the energy of incoming photons into a collective oscillation of electrons. The molar extinction coefficients of the wavelength-selective absorption and scattering of light produced is several orders of magnitude higher than typical molar extinction coefficients of organic dye molecules [90]. For example, the scattering cross-section of light from a silver nanoparticle is many orders of magnitude greater than the fluorescence cross-section of a fluorescein molecule [90]. This is the reason nanoparticles biosensors, in particular Localized Surface Plasmon Resonance (LSPR) biosensors, have emerged as a leader among label-free biosensing techniques such as labels immunoassays, surface-enhanced spectroscopies and biochemical sensors [91]. This property of nanoparticles arises from the fact that they absorb and scatter light very intensely when illuminated with light whose wavelength is equal to its Localized Surface Plasmon Resonance wavelength. The electrons in the conduction band of the nanoparticles oscillate at the resonance frequency which is dependent on the size, shape and composition of the nanoparticle. Figure 3.1 shows a schematic representation of a typical LSPR. 


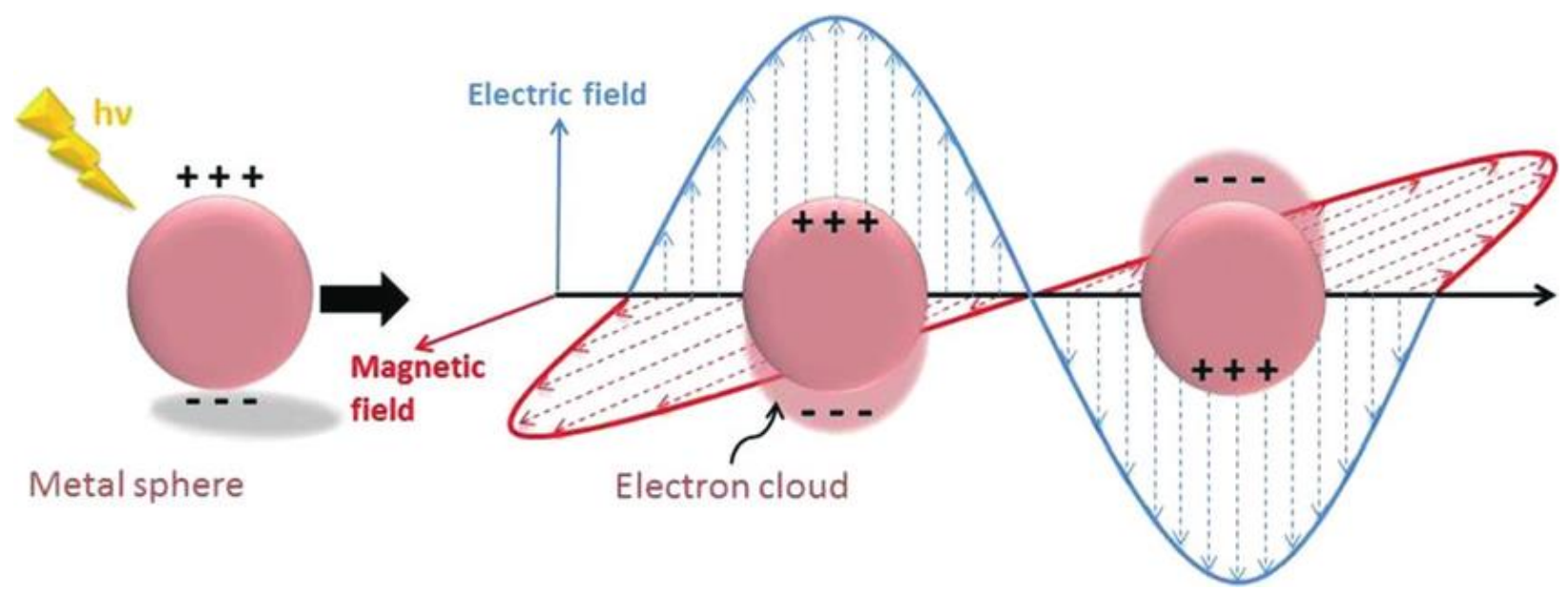

Figure 3.1: Schematic representation of a LSPR [92]

\subsubsection{Refractive Index Sensing}

Although the composition, size, shape, and the orientation of the nanoparticles contribute to the shift in the LSPR peak wavelength, the local dielectric environment of the nanoparticles also determines its peak wavelength, extinction and scattering spectral $[83,93]$. A change in the local refractive index of the nanoparticle environment can result into spectral shifts in the intense nanoparticle extinction and scattering spectra. Simple spectrometer setup can be used to measure the molecular binding in real-time with high sensitivity. This is because most organic molecules tend to increase the local refractive index which in turn causes extinction and scattering spectrum to redshift when they bind to nanoparticles [39, 47, 94-97]. The relationship between LSPR shift and changes in refractive index can also be expressed as:[91] 


$$
\Delta \lambda=m\left(n_{\text {adsorbate }}-n_{\text {medium }}\right)\left[1-\exp p^{\left(-\frac{2 d}{l_{d}}\right)}\right]
$$

Where $m$ is the sensitivity factor (in nm per refractive index unit (RIU)), $n_{\text {adsorbate }}$ and $n_{\text {medium }}$ are the refractive indices (in RIU) of the adsorbate and medium surrounding the nanoparticle, respectively, $d$ is the effective thickness of the adsorbate layer (in nm) and $l_{d}$ is the electromagnetic field decay length (in $\mathrm{nm}$ ) [91]. The shift in LSPR peak is linear to a good approximation over a small range of the local refractive index [35].

The sensitivity $m$, and the electromagnetic field decay length $l_{d}$ depend on the nanoparticle size, shape and composition of the nanoparticle. Optimizing these characteristics of nanoparticles as well as the change in the refractive index of the surrounding medium, which can be achieved using larger molecules and resonant labels, help to maximize the LSPR shifts.

\subsubsection{Molecular Sensing}

Apart from sensitivity to changes in the local refractive index of the surrounding medium, LSPR sensors can also detect molecular binding. It is important to note that there is a subtle difference between the sensitivity of nanoparticles to the bulk refractive index of the surrounding medium and the sensitivity of nanoparticles to molecular detection because molecular detection sensitivity depends on both the details of the nanoparticles surface structure and on its local environment. LSPR is sensitivity to a nanoscale region around the nanoparticle because of the rapid decay with distance from the nanoparticle associated with the electric fields enhancement. As an illustration of this fact, in the work of Unger et al., an AFM tip was used to maneuver 60nm dielectric spheres into close proximity with a gold nanocrescent. When the dielectric sphere was placed in the region 
where the electric field was enhanced, there was an average shift of $11 \mathrm{~nm}$ in the LSPR peak wavelength $[35,98]$. Figure 3.2 below illustrates this difference.

Bulk Refractive Index Sensing

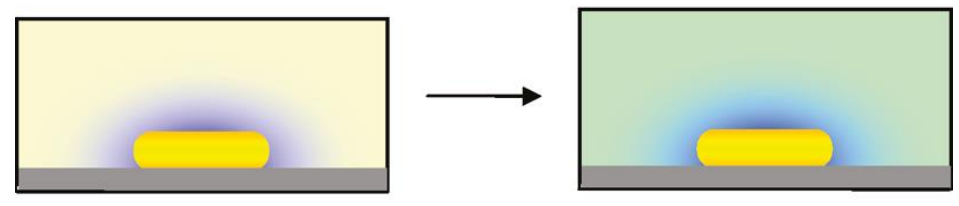

Molecular Sensing

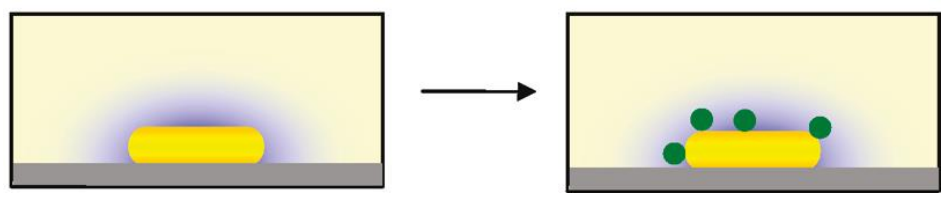

Figure 3.2: Bulk refractive index sensing versus molecular sensing by LSPR. In the case of molecular sensing by LSPR, the change to the dielectric environment of the nanoparticle is confined to the sensing volume[35] 

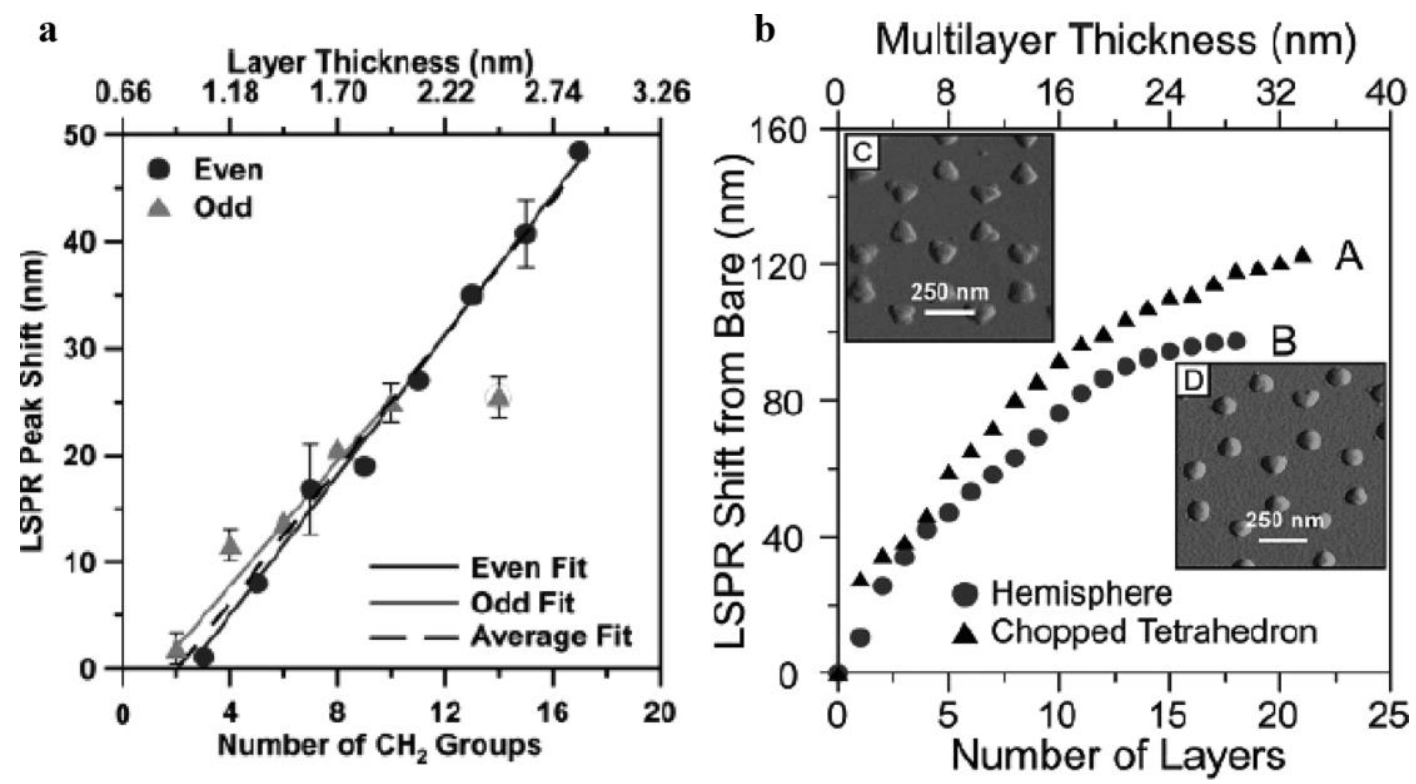

Figure 3.3: LSPR molecular detection sensitivity dependence on distance from particle surface.

(a) Detecting short SAM of varying length. (b) Detecting SAM multilayers. Copyright 2004

American Chemical Society [99, 100].

Self-assembled monolayer (SAM) formation on surface of nanoparticle is an example of molecular sensing by LSPR. Malinsky, Haes, et al. used SAM of varying carbon chain length to measure the LSPR shifts upon monolayer formation on silver nanotriangles fabricated via nonosphere lithography as the length increased one $\mathrm{CH}_{2}$ per group at a time [99-101]. They observed a decrease in the LSPR sensitivity with distance as the length of SAM was increased. A shown in Figure 3.3, they also demonstrated that SAM of varying alkane chain length (from 2 to 17 carbon atoms) varies linearly with the LSPR peak wavelength on the layer thickness up to $3 \mathrm{~nm}$ from the surface of the nanoparticle. For biosensing application, the thickness of the capture layer for SAM should be minimized so that the shift due to target binding can be observable [35].

A figure of merit for molecular detection sensitivity $\mathrm{FOM}_{\text {mol }}$ was proposed Nusz et al. They defined it as the ratio of the maximum number of bound molecules on the nanoparticle (or dynamic 
range, DR) to the minimum number of detectable molecules (or molecular detection limit, MDL). While they estimated the DR from the available surface area of the nanoparticle's, they proposed the following for the MDL: [35, 102]

$$
M D L=\frac{V_{S} \sqrt{U_{\text {system }}^{2}+U_{\text {fit }}^{2}}}{V_{A} \cdot \Delta R I \cdot e^{-2 r / l_{d} \cdot 3 S_{0}}}
$$

$$
F O M_{m o l}=\frac{D R}{M D L}
$$

where $V_{S}$ is the sensing volume, $V_{A}$ is the analyte volume, $\Delta R I$ is the refractive index difference between the analyte and the surrounding medium, $U_{\text {system }}$ is the uncertainty in the physical detection of the LSPR peak, $U_{f i t}$ is the uncertainty in the fitting of the LSPR peak, $S_{0}$ is the bulk refractive index sensitivity, $r$ is the distance from the surface of the particle to where the analyte binds, and $l_{d}$ is the decay length of the electric field from the particle surface. 
It was determined that the ideal nanorod geometry for molecular detection of streptavidin is approximately $60 \mathrm{~nm}$ by $30 \mathrm{~nm}$ with a predicted MDL of 18 , while Becker et al, proposed that the optimal nanorod aspect ratio for sensing should be between 3 and $4[35,103]$.

\subsubsection{Measurement of the Sensitivity of LSPR}

The sensitivity, $S$ of nanoparticle is expressed as the shift in the LSPR peak per unit refractive index unit $(n m / R I U)$. Since LSPR sensing depends on the shift in the peak wavelength, the sensitivity of the nanoparticles determines the precision of the shift that can be measured with respect to changes in the local refractive index. Although larger nanoparticles exhibit higher sensitivity, their peaks are broadened by multipolar excitations and radiative damping [35].

$$
S=\frac{d \lambda_{p}}{d n}
$$

Spectral linewidth, extinction intensity, electromagnetic-field strength and decay length are important plasmon characteristics to consider when dealing with the sensitivity of LSPR sensors. The sensitivity of nanoparticles to local refractive index can be enhanced by increasing the $m$ value and the electromagnetic field decay length $l_{d}$. These can be achieved by increasing the aspect ratio (width/height) of nanosphere lithography (NSL)-Fabricated nanoparticles which results in redshift in the peak wavelength, $\lambda_{\max }$. The sensitivity of LSPR can also be improved by narrowing spectral linewidths and increasing extinction [91]. 
To characterize nanoparticles sensing capabilities, a figure of merit (FOM) is used. FOM is the ratio of sensitivity to the resonance line width of the nanoparticle. Although the two debated units for FOM are $\mathrm{eV} / R I U$ and $n m / R I U$, it is important to be consistent when using any of the units for any comparison. The FOM of a nanoparticle is expressed as follows: [35, 77]

$$
F O M=\frac{S}{\Delta \lambda}
$$

The difficulty in defining a consistent LSPR line width $\Delta \lambda$ for some complex plasmonic nanostructures such as metameterials, lead to the proposal of a more generic metric for FOM* by Becker et al. They compared the sensing ability of different nanostructures in terms of the relative intensity change $d I / I$ that occurs at a given wavelength $\lambda_{0}$ as a function of a small change $d n$ to the local refractive index of the surrounding medium and excluded the line width. Their proposition makes it easier to compare sensors based on changes in intensity and those based on shift in the peak wavelength. FOM* can be expressed in terms of the refractive index sensitivity, $S$ as follows, for the latter type [35, 103].

$$
F O M^{*}=\left(\frac{\frac{d I}{d n}}{\mathrm{I}}\right)_{\max }=\left(\frac{\frac{d I}{d \lambda} \cdot \frac{d \lambda}{d n}}{\mathrm{I}}\right)_{\max }=\left(\frac{S \frac{d I}{d \lambda}}{\mathrm{I}}\right)_{\max }
$$


FOM $^{*}$ is usually defined for the wavelength that gives the maximum intensity change with refractive index.

Single-particle sensors offer improved absolute detection limits and provide better S/N resolution. They also enable higher spatial resolution in multiplexed assays and are useful for measurements in solutions, or inside cells and tissues [104-109]. The extinction of a single spherical metal nanoparticle can also be expressed as follows: [110]

$$
E(\lambda)=\frac{24 \pi^{2} N_{A} a^{3} \epsilon_{m}^{3 / 2}}{\lambda \operatorname{In}(10)}\left[\frac{\epsilon_{i}(\lambda)}{\left(\epsilon_{r}(\lambda)+2 \epsilon_{m}\right)^{2}+\epsilon_{i}(\lambda)^{2}}\right]
$$

$E(\lambda)$ is the extinction, the sum of absorption and scattering, $a$ is the radius of the metallic nanosphere, $N_{A}$ is the areal density of nanoparticles, $\epsilon_{m}$ is the dielectric constant of the medium surrounding the nanosphere, assumed to be a positive, wavelength-dependent, real number. $\epsilon_{r}(\lambda)$ is the real portion of the metallic nanosphere's wavelength-dependent dielectric function. The LSPR $\lambda_{\max }$ occurs at the wavelength where $\epsilon_{i}(\lambda)$ is small and $\epsilon_{r}(\lambda)=-2 \epsilon_{m}$, making the denominator of the term in brackets approach zero. A factor, $\chi$ which is a function of the nanoparticle aspect ratio and accounts for nanoparticle shape is introduced into equation 2 for nanospherical nanoparticles so that the LSPR occurs when $\epsilon_{r}(\lambda)=-\chi \epsilon_{m}$. Increasing the aspect ratio of nanoparticles increases $\chi$ and results in a greater refractive index sensitivity [110].

The surface area of smaller nanoparticles is very useful for protein labelling and cellular imaging because they help to reduce non-specific interactions and enables more target binding. They 
produce more confined electromagnetic fields which makes them more sensitive to single molecules which occupy a greater portion of their sensing region.

\subsubsection{Enhancing LSPR Shift}

Increasing the effective change in refractive index per molecules binding increases the sensitivity of nanoparticles. One way to achieve this is using lager molecules which produce larger LSPR shifts in proportion to the mass of the molecule $[99,100,111]$. Nanoparticle substrates that exhibit high sensitivity to changes in refractive index are used for detecting an analyte biomolecule. According to the Drude model of electronic structure of metals, plotting the LSPR frequency against the change in refractive index of the adsorbate and the surrounding medium give a linear relationship within a small range of the refractive index [35].

Another way involves plasmon resonance coupling. Chromophores that absorb visible light have been used with nanoparticles to detect small molecules binding to protein receptors. Redshift results when chromophores adsorb onto silver nanoparticles. The amount of redshift is dependent on the spectral overlap between the molecular absorbance of the chromophore and the LSPR of the nanoparticle. This sensitivity to molecular absorption makes it possible to develop highly sensitive sensors based on shift in molecular absorbance in response to analyte concentration. It is possible to detect weak changes in molecular absorption intensity or wavelength by combining indicator dyes with LSPR nanosensors. This is achieved by transducing the weak signal into spectral shift in the intense LSPR scattering and absorbance signals. However, a major setback with this method is to select and optimize the nanoparticle LSPR to match the dye resonance and to control potential interferants $[64,91,112-115]$. 
Nanoparticles also show distance-dependent coupling with other particles or surfaces displaying plasmon resonance. By sandwiching molecules between two nanoparticles, LSPR sensitivity can be exploited to detect changes in the extension of single molecules. Hence, conformational changes and binding interactions can be explored using nanoparticles as labels for proteins and ligands. The orientation of the nanoparticle pair with respect to the polarization axis of the incident light determines the magnitude and direction of the spectral shift $[83,113,114,116-124]$. The spectral shift decreases approximately exponentially with the distance between nanoparticles [119]. This distanced dependence is roughly proportional to the particle radius, such that smaller particles are more sensitive to small changes in plasmonic spacing [91].

Plasmonic coupling has greater effect on LSPR shift than changes in the local refractive index of the surrounding medium [51]. The hybridization of DNA oligonucleotides complementary to the single-stranded DNA (ssDNA) has been detected by a molecular ruler exploiting plasmonic resonance coupling between nanoparticles. Straptavidin-functionalized gold nanosphere, $40 \mathrm{~nm}$ in diameter, were immobilized on glass slides and then exposed to nanoparticles functionalized with biotinylated ssDNA. The ssDNA-functionalized nanoparticles were captured by the immobilized nanoparticles to forms pairs. The effect of plasmon resonance coupling between the nanoparticles resulted in an immediate redshift and an increase in scattering intensity of the immobilized nanoparticles. These plasmonic rulers have been used to measure dynamic biophysical processes $[91,113]$. 


\section{Chapter 4: Design Layout of Real-Time Plasmonic qPCR Instrument}

As shown in figure 4.1, our plasmonic qPCR instrument design is based on the same principle as that by Roche et al. [125] with some modifications. The following sections describes the three main components of this instrument: the thermocycling, the sensing part and the control system.

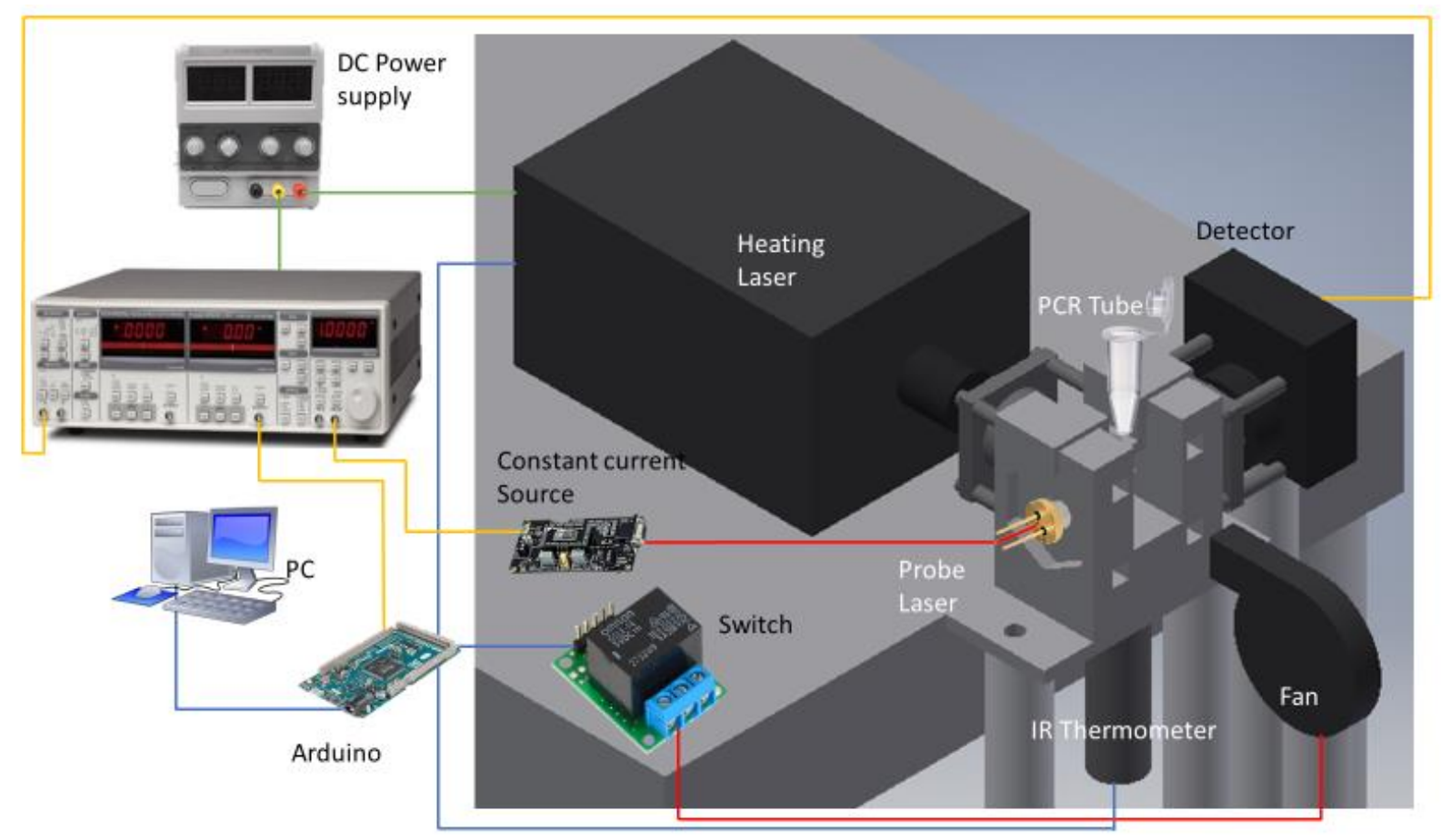

Figure 4.1: Design Layout of Real-Time Plasmonic qPCR System

\subsection{Optical Thermocycling Instrument Design}

PCR requires thermocycling between different temperatures from denaturation, to annealing, and then to elongation. This optical thermocycling instrument design, exploits the photothermal property of nanoparticles to provide the heat needed for thermocycling in PCR. Nanoparticles under excitation with light at the resonance wavelength, generate oscillating dipole. The energy 
generated from this oscillation is dissipated through ohmic heating losses to raise the temperature of the surrounding medium. [126-128]. Thus, each nanoparticle is exploited as a nano-heater for to heat the PCR solution. Equation 4.1 models the heating effect of a colloidal solution of nanoparticles [129]. Although the nanoparticle can either be in direct contact or indirect contact with the reaction mixture, it is preferable to use the direct contact method since it improves on the heating efficiency of the nanoparticle on the PCR mixture. The heating efficiency is determined by the aspect ratio of the GNRs. Additionally, the concentration of the nanoparticles in the reaction mixture directly affects the heating efficiency according to formula:

$$
m C_{p} \frac{d T}{d t}=P \frac{\varepsilon_{a b s}}{\varepsilon_{e x t}}\left(1-10^{-A_{\lambda}}\right) \eta-h S\left(T-T_{\text {ambient }}\right)
$$

where $A_{\lambda}=\varepsilon_{\text {ext }} c l$ is the absorbance, $m$ is the mass of the solution, $C_{p}$ is the specific heat capacity of the solution, $T$ is the solution temperature, $t$ is the time, $P$ is the power of the incident laser, $\varepsilon_{a b s}$ is the molar absorption coefficient, $\varepsilon_{\text {ext }}$ is the molar extinction coefficient, $\eta$ is the power conversion efficiency of the nanoparticle, $h$ is the heat transfer coefficient between the solution and surrounding, $S$ is the surface area separating the solution from the surrounding, and $T_{\text {ambient }}$ is the ambient temperature.

The steady state solution for the differential equation above for a fixed laser output power is given as [130]: 


$$
T=P \frac{\varepsilon_{a b s}}{\varepsilon_{\text {ext }}}\left(\frac{1-10^{-A_{\lambda}}}{h S}\right) \eta-T_{\text {ambient }}
$$

The optical thermocycling part consists of an $800 \mathrm{~nm}(5000 \mathrm{~mW})$ LRD-0808 collimated diode laser system purchased from Laserglow Technology. The laser is set to operate at $2 W$ and the collimated beam from this laser is focused onto a $0.2 \mathrm{ml}$ thin wall PCR strip tube with a Thorlabs' LA1951-B-ML N-BK7 plano-convex lens, with $25.4 \mathrm{~mm}$ focal length and $25.4 \mathrm{~mm}$ diameter. The focused beam diameter is adjusted to cover the area of the PCR strip tube containing the PCR mix and gold nanorod solution for effective power control and efficient heating for optimal PCR product. A $12 \mathrm{~V}$ DC brushless fan from Digi-Key Electronics connected to 5DVC relay (SRD05VDC-SL-C) is used to provide cooling for the PCR system. An infra-red thermometer from Optris, placed $10 \mathrm{~mm}$ from the PCR strip tube, is used to record the temperature of the PCR and gold nanorods solution. The light from the heating laser is used to excite the gold nanorods since the wavelength of the incident light is the same as the longitudinal resonance wavelength of the gold nanorods in the solution. This causes the gold nanorods to generate the heat needed for thermocycling.

The rate of energy transfer to the nanoparticle and consequently, the temperature of the PCR reaction mixture can be controlled by varying the intensity of the $800 \mathrm{~nm}$ laser beam and the exposure time of the nanoparticles to the beam. Hence, rapid thermocycling between different set 
temperatures is achieved by the excitation of GNRs in the reaction mixture through controlled pulsing of the $800 \mathrm{~nm}$ laser, accompanied by a cooling system.

\subsection{LSPR Optical Sensing Instrument Design}

The optical sensing component of the LSPR qPCR instrument is required to measure the change that occurs during the PCR process. In principle, as PCR progresses from the initial cycle to the final cycle, DNA is amplifier exponentially and hence, the concentration of DNA in the PCR solution increases because of this amplification. This alters the optical properties of the PCR mix medium surrounding the gold nanorods. The change in the optical properties of the medium results in a corresponding shift in the resonance wavelength of the nanoparticles. Experimental results obtained from this system suggests a decrease in transmission of light at $670 \mathrm{~nm}$ as the concentration of DNA increases.

The optical sensing instrument consists of a $10 \mathrm{~mW}, 670 \mathrm{~nm}$ laser diode that provides the light used to probe the PCR-gold nanorod solution. This wavelength of light is so chosen to exploit the lowest absorbance point between the transverse and longitudinal resonances of the $808 \mathrm{~nm}$ gold nanorods for sensing [125]. The probe laser is powered from a constant current source to keep the output power of the laser constant over the period of the PCR cycle. An optical shortpass filter (FES0750) with $750 \mathrm{~nm}$ cut-off wavelength is used to filter out noise from wavelengths of light other than $670 \mathrm{~nm}$. This includes noise from the heating laser and other light sources in the surrounding. The filtered light is then focused onto a PDA34 silicon amplified photodetector, used to measure the transmitted light through the solution on the opposite end of the PCR strip tube. The output from the photodetector is connected to a SR830 DSP lock-in amplifier (Stanford Research Systems) modulated by a sine wave at $1000 \mathrm{~Hz}$ to further filter out noise. The 
laser/photodetector system and the heating laser system were aligned at $90^{\circ}$ to each other on a $3 \mathrm{D}$ plastic holder as shown in figure 4.1. Apart from the heating laser, all other components were purchased from Thorlabs.

\subsection{The Control System}

The control operation is handled by a programmed Arduino Due microcontroller which provides the digital proportional control used to: modulate the heating laser, control the fan, keep track of the temperature of the PCR solution from the IR thermometer during the thermocycling process and record the transmission signal from the lock-in amplifier in real-time. The output data of the Arduino Due which consists of time, the temperature of the solution, cycle number and the transmission output from lock-in amplifier is then recorded and displayed via the serial monitor.

\subsection{Choice of Gold Nanorods}

As predicted by Gans's theory, gold nanorods which are commonly treated as ellipsoids, given a fixed geometric parameter, show a redshift in the resonance wavelength of the longitudinal mode in a medium with increasing dielectric constants [87].Gold nanorods (GNRs) have both lateral and axial dimensions that give rise to corresponding transverse and longitudinal plasmon resonance wavelengths. GNRs have greater extinction coefficients than gold nanospheres and they produce more heating power per unit of laser power incident on the nanoparticle. This property makes gold nanorods a preferred choice to gold nanospheres in simple, fast and sensitive LSPR biosensing application.

To explain the reason why GNRs were used instead of carbon nanotubes, the maximum optical density (OD) per unit length for single-wall and multi-wall carbon nanotube (SWCNT) without PCR inhibition was determined to be $0.144 \mathrm{~mm}^{-1}$ and $0.0446 \mathrm{~mm}^{-1}$ respectively, while the 
maximum OD per unit length for gold nanorods without PCR inhibition was $3.05 \mathrm{~mm}^{-1}$. By implication, the plasmonic heating of GNR and CNTs, show that GNRs are almost 21-times more absorptive of light and generate more heat than SWCNTs and 68-times more absorptive and heat generating than MWCNTs. Hence GNRs are more preferred to CNTs.

The maximum absorption of the longitudinal plasmon resonance wavelength shows a linear dependence on the aspect ratio (defined as the longitudinal axis divided by the diameter in nanometers) of GNRs [87]. For example, GNRs with diameters $10 \mathrm{~nm}$ and 20 $\mathrm{nm}$, that with a smaller diameter $(10 \mathrm{~nm})$ corresponding to a higher aspect ratio, would provide a better heating efficiency than that with larger diameter or a lower aspect ratio. This property is important in optimizing the heating efficiency of GNRs used in thermocycling. Hence, by using a particle with a large absorption cross-section and a corresponding high extinction coefficient, the heating efficiency is maximized.

GNRs are modified with Polyethylene glycol (PEG) or any other chemical with similar property of preventing the inhibition of a positive active site of polymerase class enzymes. The PEG-GNR can be used as generic heaters irrespective of the type of polymerase, size of the DNA template or the type of DNA template. As PCR proceeds, amplified DNA products bind to the surface of GNRs thereby changing the dielectric constant around the nanoparticles and hence, induce a change in the resonant absorbance and spectral position. A probe laser is then used to measure the change in absorption at a particular wavelength.

In this work, we used GNRs functionalized with PEG (also called PEG-GNR) with $811 \mathrm{~nm}$ (this was the closest to $808 \mathrm{~nm}$ available) longitudinal resonance wavelength, $513 \mathrm{~nm}$ transverse 
wavelength, $78 \mathrm{nM}$ concentration, $9.8 \times 10^{8} \mathrm{M}^{-1} \mathrm{~cm}^{-1}$ Molar Extinction, and 4.1 aspect ratio (10 $\mathrm{nm}$ diameter and $41 \mathrm{~nm}$ length) from Nanopartz. 


\section{Chapter 5: Experimental Procedures/ Description of Experiments}

\subsection{Investigating Potential Inhibition of GNRs to PCR}

The concern that polymerases are known to interact with gold nanoparticles by electrostatic adsorption to the surface of the particle thereby blocking the active site and preventing DNA polymerization, led to the investigation of the potential inhibition of GNRs to PCR reaction. The aim was to determine the concentration required for optimum heating and non-inhibition of PCR. A dilution series was prepared from the stock GNRs by replacing water with increasing volumes of gold nanoparticles in the aqueous solution. Each PCR mixture contains increasing concentration of GNRs. Eppendorf thermocycler was used to perform the PCR reactions.

\subsubsection{Inhibitory concentration of bare gold nanorods (GNR).}

Dilution series was carried out for $1 n M$ stock concentration of non-functionalized (or bare) GNR. The GNRs were washed and concentrated; thereafter, they were diluted from $3 n M$ to $0.2 n M$. In a total volume of $20 \mu \mathrm{l}$ PCR reaction, $9 \mu \mathrm{l}$ of GNRs was added to the mixture. A control PCR without GNR was also performed. It was observed that PCR was inhibited at $0.5 n M$ concentration of nonfunctionalized GNRs. The results from the PCRs was visualized through gel electrophoresis using $1 \%$ agarose gel as shown in figure 5.1. It is obvious from the result that although bare GNRs can be used in PCR reactions, any concentration greater than $0.5 n M$ would be inhibitory to the PCR reaction. 


\section{$\begin{array}{llllllllll}M & 3 & 2 & 1 & 0.5 & 0.1 & 0.05 & 0.02 & 0 & n M \\ n M & \text { "bare" GNR }\end{array}$}

\section{$\Leftrightarrow \ldots$}

Figure 5.1. Gel result for non-functionalized GNRs inhibitory test

\subsubsection{Inhibitory concentration of PEGylated GNR.}

Dilution series was carried out for $1 n M$ stock concentration of non-functionalized (or bare) GNR. The GNRs were washed and concentrated or diluted from $1 n M$ to $100 n M$. In a total volume of $20 \mu l$ PCR reaction, $9 \mu l$ of GNRs were added to the mixture. A control PCR without GNR was also performed. It was observed that PCR were inhibited at 100nM concentration of PEG-GNRs. The results from the PCRs was visualized through gel electrophoresis using 1\% agarose gel as shown in figure 5.2. It is obvious from the result that PEG-GNRs can also be used in PCR reactions however, extremely high concentration of PEG-GNRs (greater than 50nM) would be inhibitory to the PCR reaction.

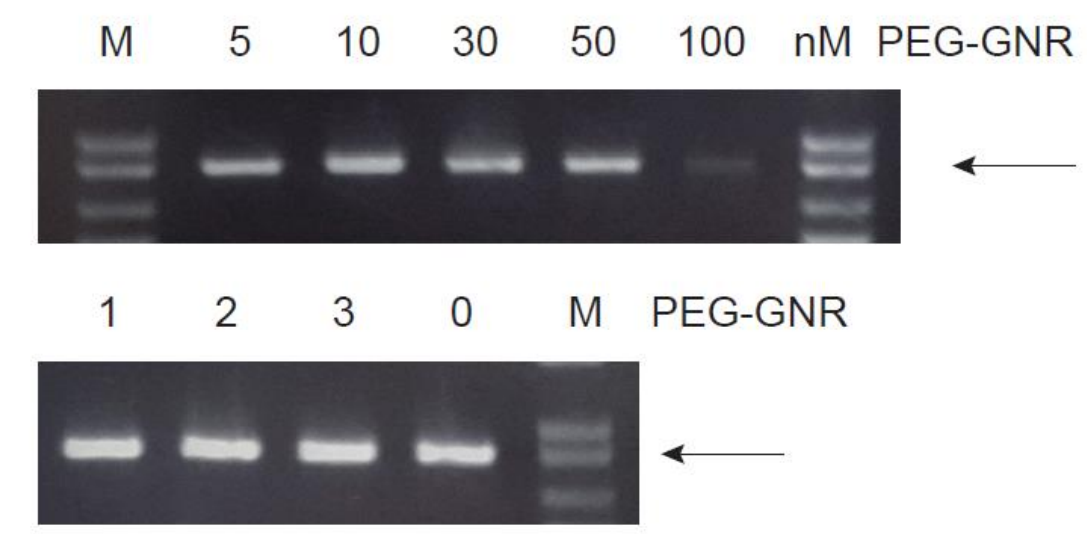

Figure 5.2: Gel result for PEG-GNRs inhibitory test 


\subsubsection{Effect of CTAB on PCR reaction.}

PEG-GNR particles were prepared in $1 \% \mathrm{CTAB}$ or washed and resuspended in water, at different concentration (20nM or $30 n M)$. In a total volume of $20 \mu l$ PCR reaction, $9 \mu l$ of GNRs were added to the mixture. A control PCR without GNRs was also performed. It was observed that PCR was inhibited at $100 \mathrm{nM}$ concentration of PEG-GNRs. The results from the PCRs was visualized through gel electrophoresis using $1 \%$ agarose gel as shown in figure 5.3. It is obvious from the result that there was no inhibition or toxicity to the PCR reaction from the presence of $\mathrm{CTAB}$ in the reaction mixture. Hence, the presence of CTAB does not inhibit the PCR reaction.

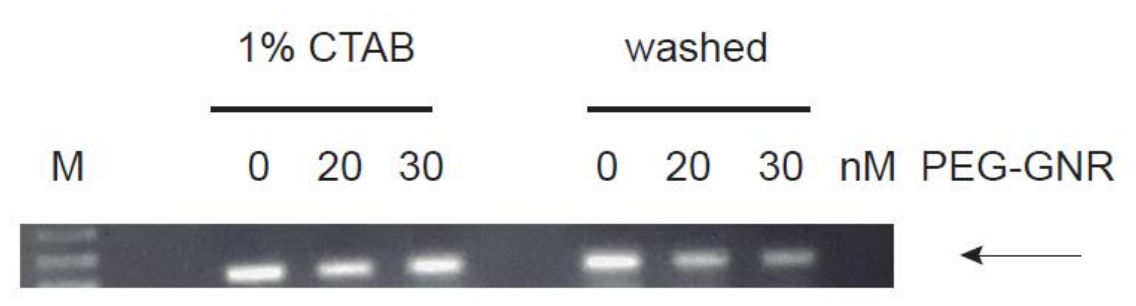

Figure 5.3: Gel result for CTAB inhibitory test

\subsection{Plasmonic PCRs Using PEG-GNRs}

After the inhibitory effect of gold nanoparticles in PCR was investigated, experiments were carried out using PEG-GNR in the plasmonic system. The PEG-GNRs and PCR reaction mixture were placed within a $0.2 \mathrm{ml}$ thin wall PCR strip tubes. The mixture was covered with $50 \mu l$ of mineral oil and capped to prevent evaporation. The constituents of the PCR reaction mixture are as shown in table 5.1. 


\begin{tabular}{|l|l|l|}
\hline Components of Positive PCR & Components of Negative PCR & $\begin{array}{l}\text { Volume } \\
(\mu l)\end{array}$ \\
\hline 5 X Phusion HF Reaction Buffer (NEB) & 5 X Phusion HF Reaction Buffer (NEB) & 4 \\
\hline dNTPs (10nM) (NEB) & dNTPs (10nM) (NEB) & 0.5 \\
\hline Forward Primer & Forward Primer & 1 \\
\hline Reverse Primer & Reverse Primer & 1 \\
\hline DNA & $\mathrm{d}_{2} \mathrm{H}_{2} \mathrm{O}$ & 2 \\
\hline Klen Taq Polymerase & Klen Taq Polymerase & 1.6 \\
\hline PEG-GNR (808nm) & PEG-GNR (808nm) & 1 \\
\hline $\mathrm{d}_{2} \mathrm{H}_{2} \mathrm{O}$ & $\mathrm{d}_{2} \mathrm{H}_{2} \mathrm{O}$ & 8.9 \\
\hline Total & Total & 20 \\
\hline
\end{tabular}

Table 5.1: Volume of Components of Positive and Negative PCR Reactions Mixture

The final volume of the GNRs-PCR mixture was $20 \mu l$ and a total of $70 \mu l$ volume of GNR-PCR mixture and mineral oil were contained in the PCR tube. The reaction tube was placed in a plastic holder which also houses the IR thermometer, $670 \mathrm{~nm}$ probe laser, fan, and photodetector; and provides the path for focused light from the $808 \mathrm{~nm}$ heating laser to irradiate the reaction tube. 
The cooling fan and heating laser were controlled through an Arduino microcontroller interface. The IR thermometer was placed $10 \mathrm{~mm}$ from the bottom of the tube secured in the plastic holder, to record the temperature via the microcontroller.

The temperature for each stage of the reaction and the time to maintain each set temperature are set via the Arduino microcontroller graphical interface. A USB link transmits the information from the microcontroller to the computer. A negative feedback control system is established with the help of the set temperatures and times to actuate the cooling fan and dynamically pulse the heating laser depending on the temperature required and the stage of the reaction cycle. The temperature curve showed a good stability during plasmonic thermocycling. The denaturation (85.0 \pm $\left.0.11^{\circ} \mathrm{C}\right)$, annealing $\left(60.0 \pm 0.09^{\circ} \mathrm{C}\right)$, and elongation $\left(72.0 \pm 0.13^{\circ} \mathrm{C}\right)$ showed an overall accuracy of $0.1^{\circ} \mathrm{C}$.

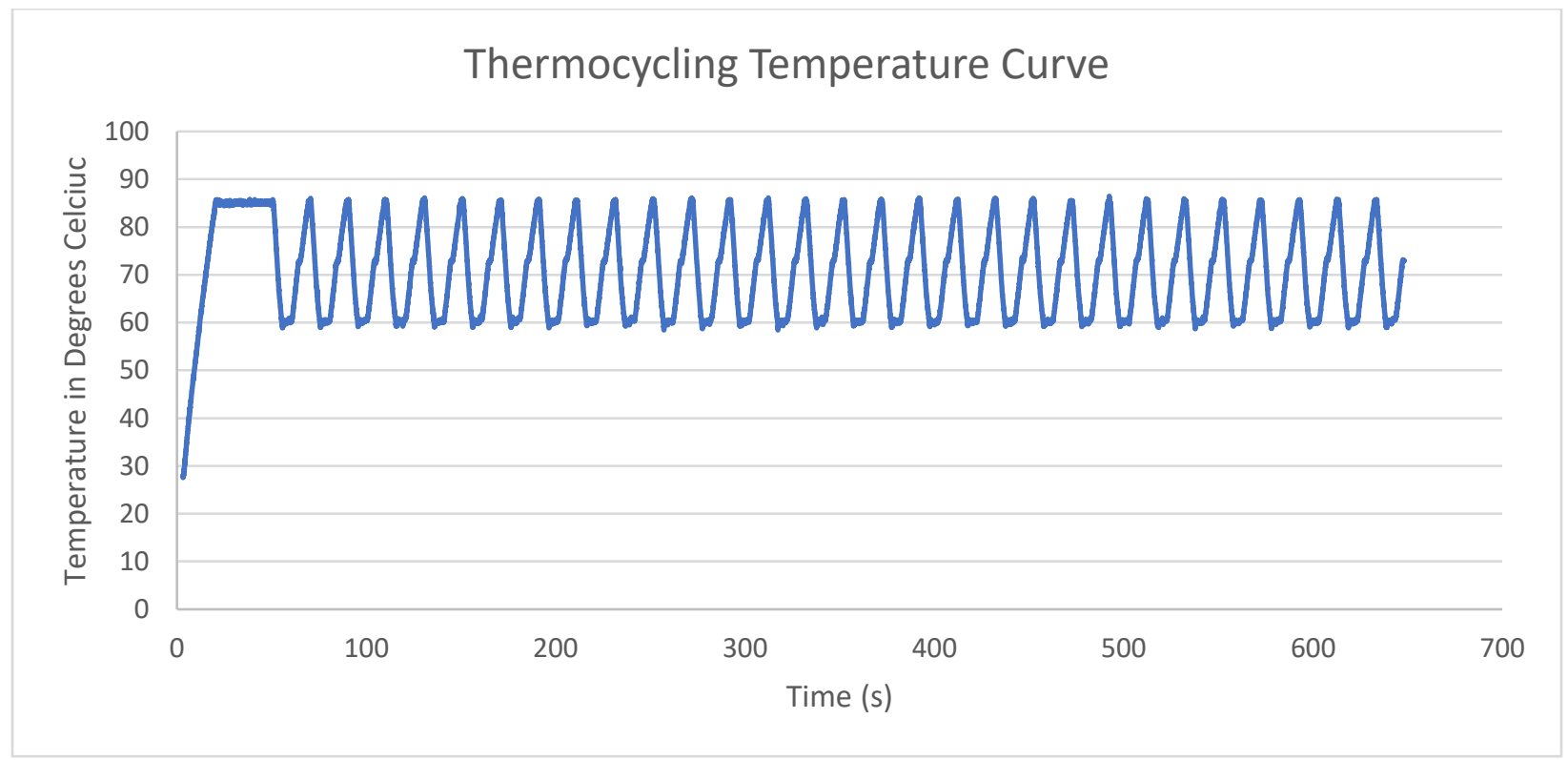

Figure 5.4: Thermocycling Temperature Curve for 30 Cycles 
Figure 5.6 shows the thermocycling achieved through plasmonic heating. It took just over 10 minutes of total reaction time to complete the experiment. Following the temperature curve in figure 5.6, the reaction process can be understood. First, the temperature of the PCR mixture was raised from room temperature to $85.0^{\circ} \mathrm{C}$ to allowe for the denaturation of DNA for 30 seconds. This is the hot-start stage. Apart from this first denaturation stage otherwise know as the hot-start phase, subsequent denaturation stages were allowed for just 1 second. Once the denaturation phase is completed, the heating laser is turned off and the fan is activated to allow for the cooling of the PCR mixture. Hence, the temperature of the PCR mix is lowered to $60.0^{\circ} \mathrm{C}$ at the annealing stage. This temperature is held at this stage for 5 seconds by pulsing the heating laser to compensate for the temperature difference through the feedback system of the microcontroller. After the annealing stage is completed, the elongation phase sets in and the fan is turned off. The laser is turned on and the nanoparticles heat up the PCR solution from the annealing temperature to $72.0^{\circ} \mathrm{C}$ which is the elongation temperature. At this temperature the temperature is held for 1 second by pulsing the laser as described for the annealing phase above. The process is repeat for 30 Cycles.

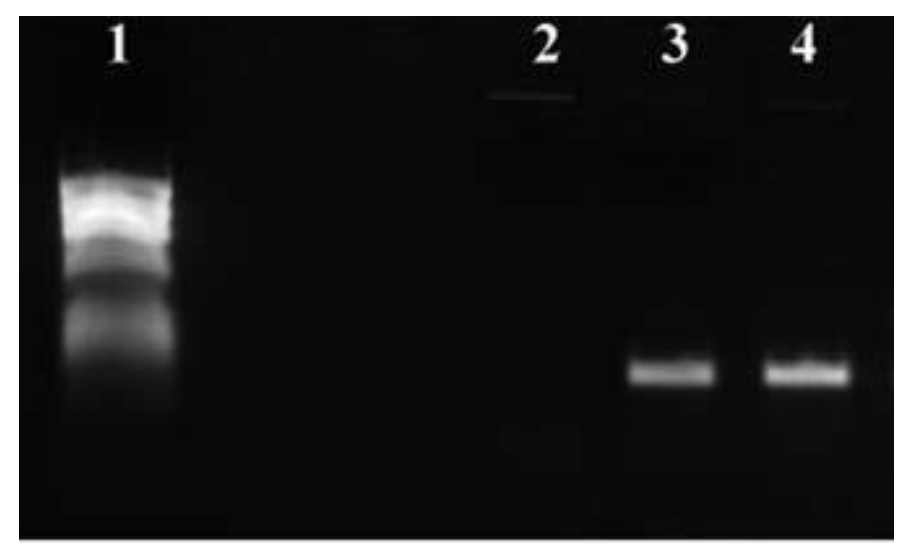

Figure 5.5: Gel Electrophoresis Results for Plasmonic PCR 
The product of the PCR is separated on a 1.5\% agarose gel by electrophoresis. Figure 5.7 show the picture of a gel result. Lane 1 contains the size marker and lane 2 is the negative control without DNA while lane 3 and 4 contain positive controls.

In conclusion, the success of a PCR reaction depends on the type and concentration of the nanoparticles used. PEG nanoparticles are less inhibitory to PCR than bare nanoparticles, and above a certain concentration, both PEG and bar nanoparticles become inhibitory to the PCR reaction. The gel results of the PCR reaction produced by our plasmonic system shows a very robust product comparable to those of conventional PCR machines 


\section{Chapter 6: Real-Time Monitoring of DNA amplification}

Real-time monitoring of PCR reaction to detect DNA amplification involves the irradiation of the reaction mixture with a probing laser light beam with wavelength different from the resonant wavelength. This is chosen to isolate the spectral impact of the probe laser from the photothermal properties of the nanoparticle at the resonant wavelength. Since GNRs are used, the longitudinal resonance wavelength of the nanoparticle is exploited for heating the PCR while a wavelength between the transverse and longitudinal resonance wavelengths is chosen for monitoring. Hence, $808 \mathrm{~nm}$ laser was chosen to excite the GNRs for heating purpose while $670 \mathrm{~nm}$ was chosen as the wavelength of the probing light beam for monitoring.

An infra-red thermometer is used to optically monitor the change in temperature of the reaction mixture as it heats up through optical excitation of the GNRs. If well calibrated, a probe laser can also be used for temperature monitoring. Since the optical properties of nanoparticles change with respect to the change in temperature of the surrounding medium, another way to monitor the temperature of the reaction mixture would involve the use of probe laser with a wavelength different from the resonance wavelength of the nanoparticles to monitor the change in the scattering or absorbance of the nanoparticles.

The amount of DNA amplification can be monitored in real time by measuring the intensity of the transmitted probe laser light through the solution using a photodetector. Nanoparticle of resonance wavelength different from that used in the photothermal heating of the solution can also be used in the reaction mixture for monitoring the amplification process. A photodetector is used to monitor the transmitted light resulting from the interaction of the probe laser beam with the 
monitoring nanoparticles. This is then sent to a lock-in amplifier for filtering at $1000 \mathrm{~Hz}$. The filtered result is then sent to the Arduino microcontroller for processing and analysis. Figure 6.1 shows the temperature and transmission curve for a PCR experiment.

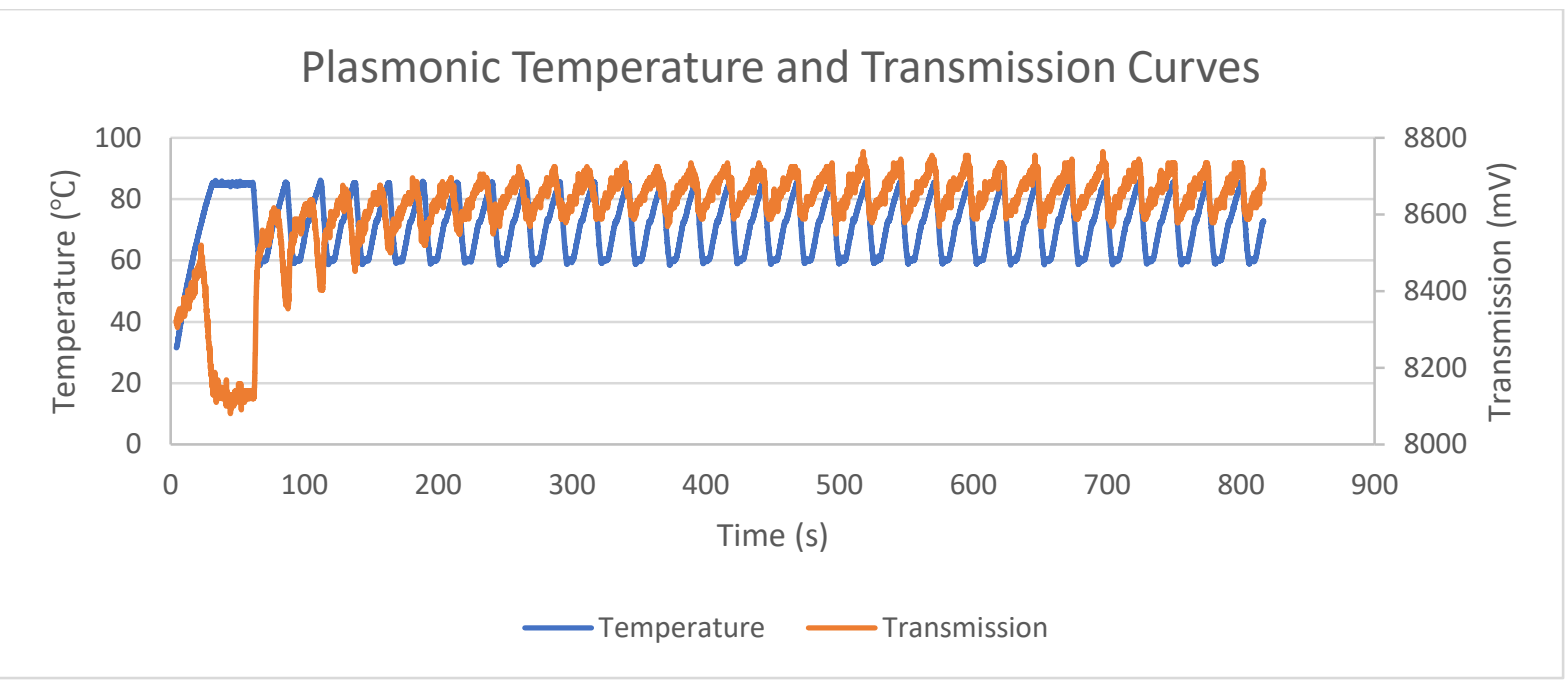

Figure 6.1: Plasmonic Temperature and Transmission Curves

The data from the temperature and transmission readings are saved in excel file for further analysis using Matlab.

\subsection{DNA Concentration Versus Transmitted Light Intensity}

The intensity of the transmitted light is proportional to the absorption of the nanoparticle which varies as the amount of DNA in the reaction mixture. A simple experiment to determine how the intensity of the transmitted light varies as the concentration of DNA was carried out. In this experiment, $2 \mu l$ of $4.5 \times 10^{6}$ copies $/ \mu l$ of genomic DNA was mixed with $18 \mu l$ of $2.5 \mathrm{nM}$ of GNRs in a PCR strip tube and the transmitted light through the solution was monitored through a photodetector and lock-in amplifier system. The genomic DNA was diluted to different fractions of the initial concentration in the GNRs solutions and the transmissions were recorded. To avoid 
reading errors arising from measuring from different sides of the PCR strip tube, the experiment was carried out in one tube without replacement. The dilution of the DNA to different concentrations was done in the same tube without moving it. This helps to mimic real PCR except that other components of the PCR apart from DNA were not present in the solution.

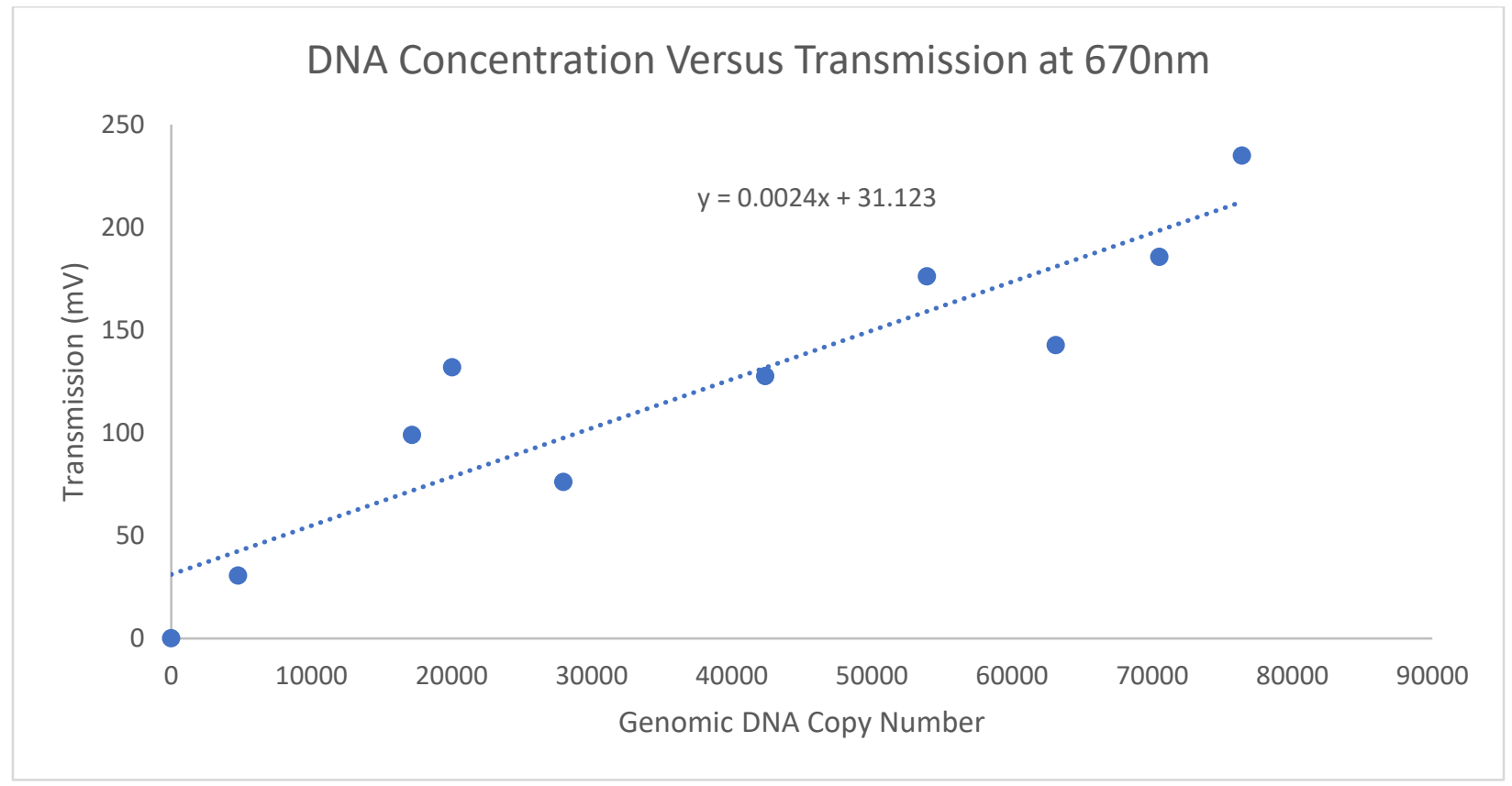

Figure 6.2: DNA Concentration Versus Transmission at 670nm

Figure 6.2 shows a linear relationship between the concentration of DNA and the transmitted light. The equation of the straight line fit for the data, is given as:

$$
\text { Transmission }=(0.0024 \times \text { Copy Number })+31.123
$$

This equation predicts that there would be a $0.24 \%$ increase in the transmission signal for every 100 copies of genomic DNA replicated. This means that since PCR is an exponential process and 
taking into account the $1 \mathrm{mV}$ noise level of our detection system, our plasmonic PCR system is capable of detecting at least 10,000 copies of DNA during amplification in real time.

Apart from the concentration of the DNA, the temperature of the PCR mixture has a small effect on the plasmonic absorption of the gold nanoparticles. This is so because dephasing of the surface plasmon electron motion is caused by electron-electron repulsion rather than electron-phonon interaction. Previous report shows that there is a very small decrease in the absorbance of nanoparticles at elevated temperature [71]. This implies that the transmission through the PCR solution increases slightly with increase in temperature. Hence, the amount of light that gets to the photodetector is slightly increased at high temperatures. Also, the refractive index of the PCR solution changes with temperature and results in a shift in the absorption spectrum of the nanoparticles. Given the change in refractive index of the solution with respect to temperature, it is possible to estimate the shift in resonance wavelength with respect to temperature using equation 3.29 . 


\section{Chapter 7: Results and Discussion}

\subsection{Amplitude Monitoring: Ratio of Transmission at Denaturation to Transmission at Annealing}

To investigate the difference between the transmission signals of positive and negative PCRs, the transmission at the end of the denaturation and annealing stages were recorded separately for a period of $40 \mathrm{mS}$ and the average for each cycle was taken. At this point, the heating laser and the cooling fan were turned off except the probe laser and lock-in amplifier detection system. The rationale behind this was to eliminate errors in the transmission signal resulting from interference with the heating laser and the cooling fan. Figure 7.1 shows the section of the transmission curve extracted after denaturation and annealing. The ratio of the transmission immediately after denaturation to the transmission immediately after annealing was computed for each cycle. Figure 7.2 shows a plot of the ratios against the number of cycles. The slope of the curves for different experiments were computed for negative PCRs and positive PCRs of different starting concentrations. Figure 7.3 shows a box and whisker plot of the results obtained from the computations. The average slope of the transmission was also computed for the different experiments. And from the results, it is obvious that PCR experiments with average slopes greater than 0.0015 would be positive PCR. In order words, for any PCR experiment, if the ratio of the transmission just after denaturation to the transmission just after annealing increases by a factor greater than 0.0015 for each increase in cycle then, it would most like be a positive PCR product otherwise, it is a negative PCR. Although, this approach could distinguish between negative and positive PCRs, it could not discriminate between the different starting concentrations. 


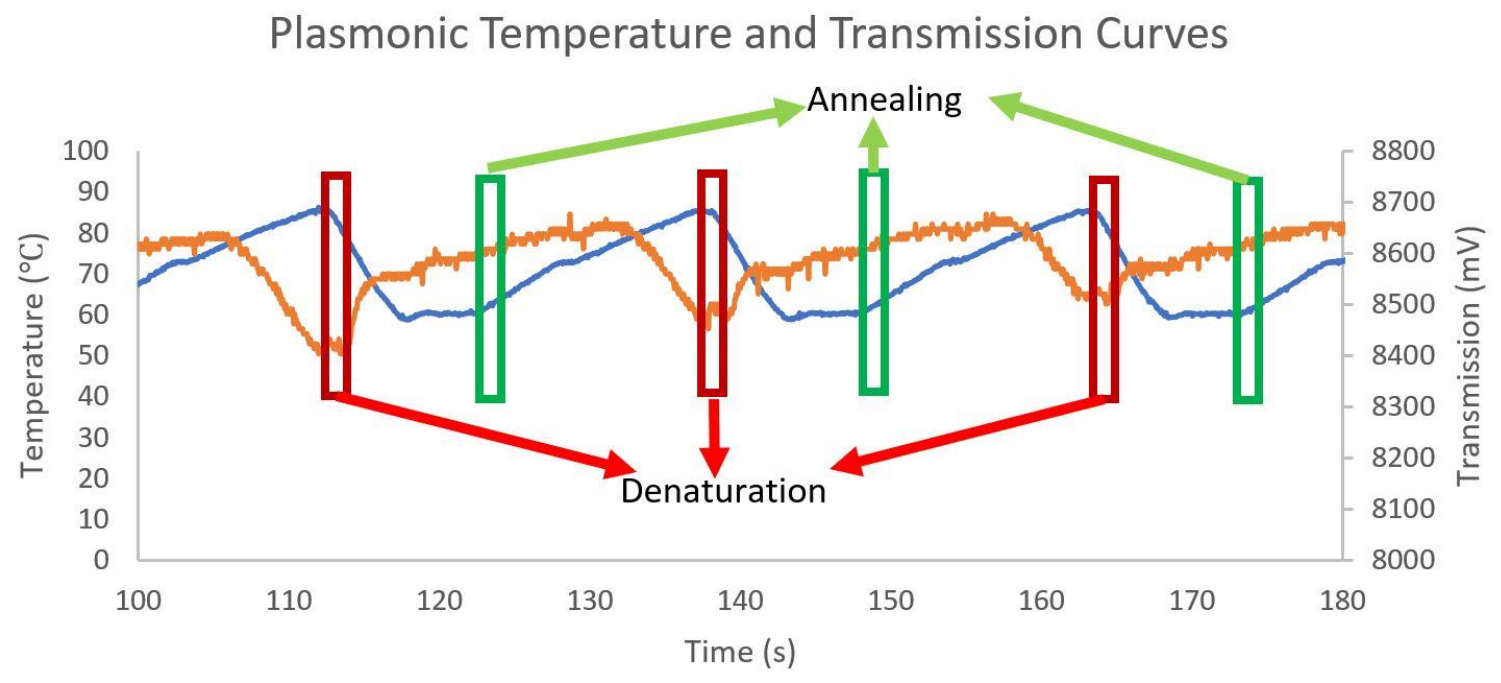

- Temperature Transmission

Figure 7.1: Section of the Transmission curve extracted for denaturation and annealing

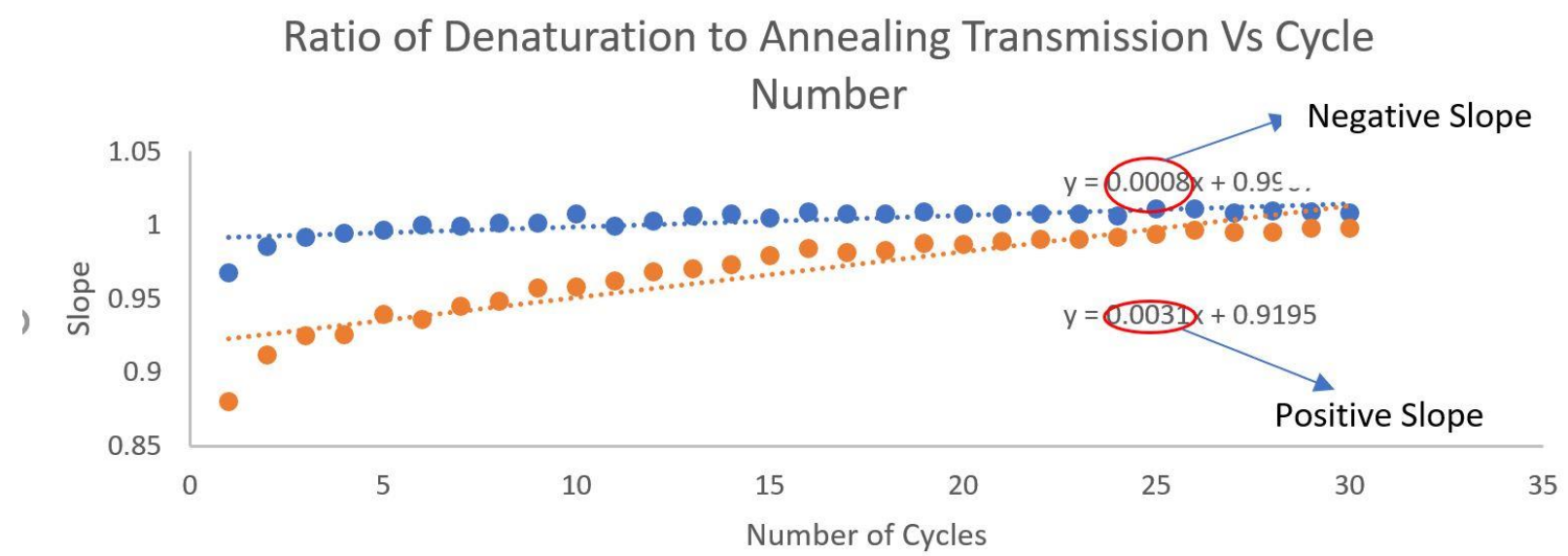

- Negative Positive ........ Linear (Negative) ......... Linear (Positive)

Figure 7.2: Plot of the ratio of Denaturation to Annealing Transmission Vs Cycle Number showing the Slopes of the Positive and Negative PCR Curves 


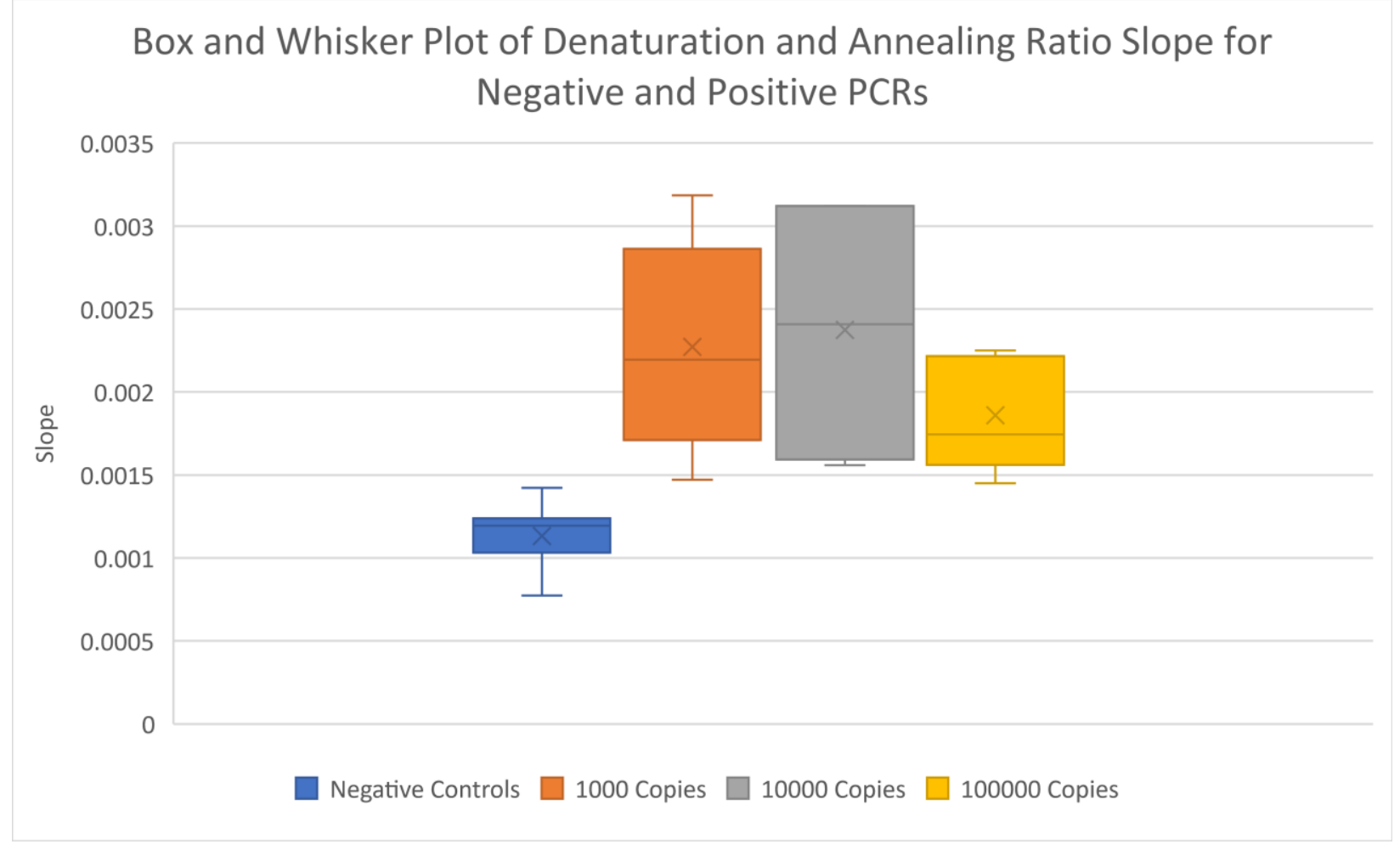

Figure 7.3: Box and Whisker Plot of Denaturation and Annealing Ratio for Negative Controls and Varying concentrations of DNA

\subsection{Amplitude Monitoring: Ratio of Transmission at Denaturation to \\ Transmission at Elongation}

We explored the ratio between denaturation and elongation for different cycle number to further investigate the difference between the transmission signals of positive and negative PCRs. Similar to the approach described in section 7.1, the transmission at the end of the denaturation and elongation stages were recorded separately for a period of $40 \mathrm{mS}$ and the average for each cycle was taken. At this point, the heating laser and the cooling fan were turned off except the probe laser and lock-in amplifier detection system. This was done to eliminate errors in the transmission signal resulting from interference with the heating laser and the cooling fan. Figure 7.4 shows the 
section of the transmission curve extracted after denaturation and elongation. The ratio of the transmission immediately after denaturation to the transmission immediately after elongation was computed for each cycle. Figure 7.5 shows a plot of the ratios against the number of cycles. The slope of the curves for different experiments were computed for negative PCRs and positive PCRs of different starting concentrations. Figure 7.6 shows a box and whisker plot of the results obtained from the computations. The average slope of the transmission was also computed for the different experiments. And from the results, it is obvious that PCR experiments with average slopes greater than 0.0015 would be positive PCR. In order words, for any PCR experiment, if the ratio of the transmission just after denaturation to the transmission just after elongation increases by a factor greater than 0.0015 for each increase in cycle then, it would most like be a positive PCR product otherwise, it is a negative PCR. Although, this approach could distinguish between negative and positive PCRs, it could not discriminate between the different starting concentrations.

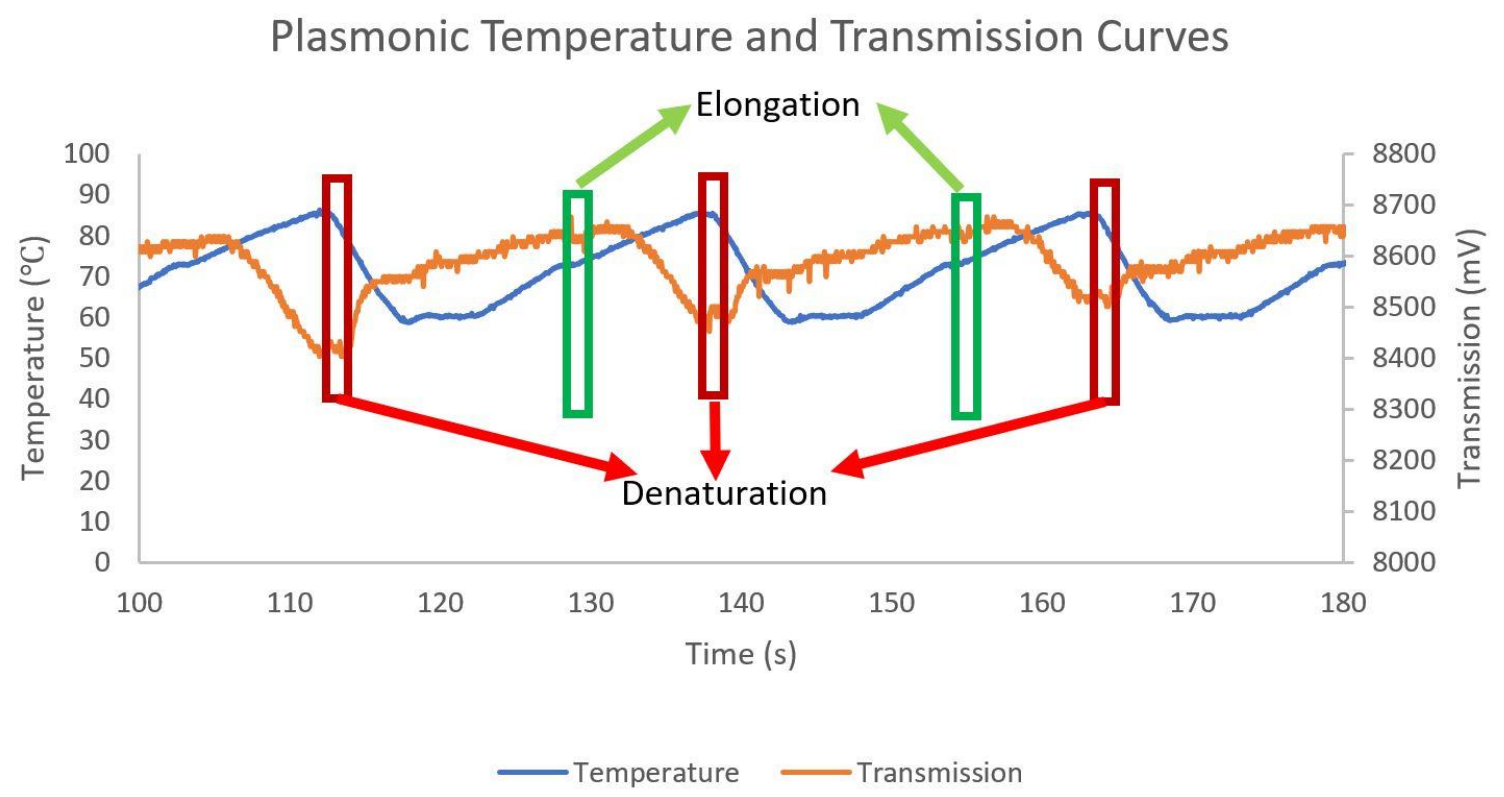

Figure 7.4: Section of the Transmission curve extracted for denaturation and Elongation 


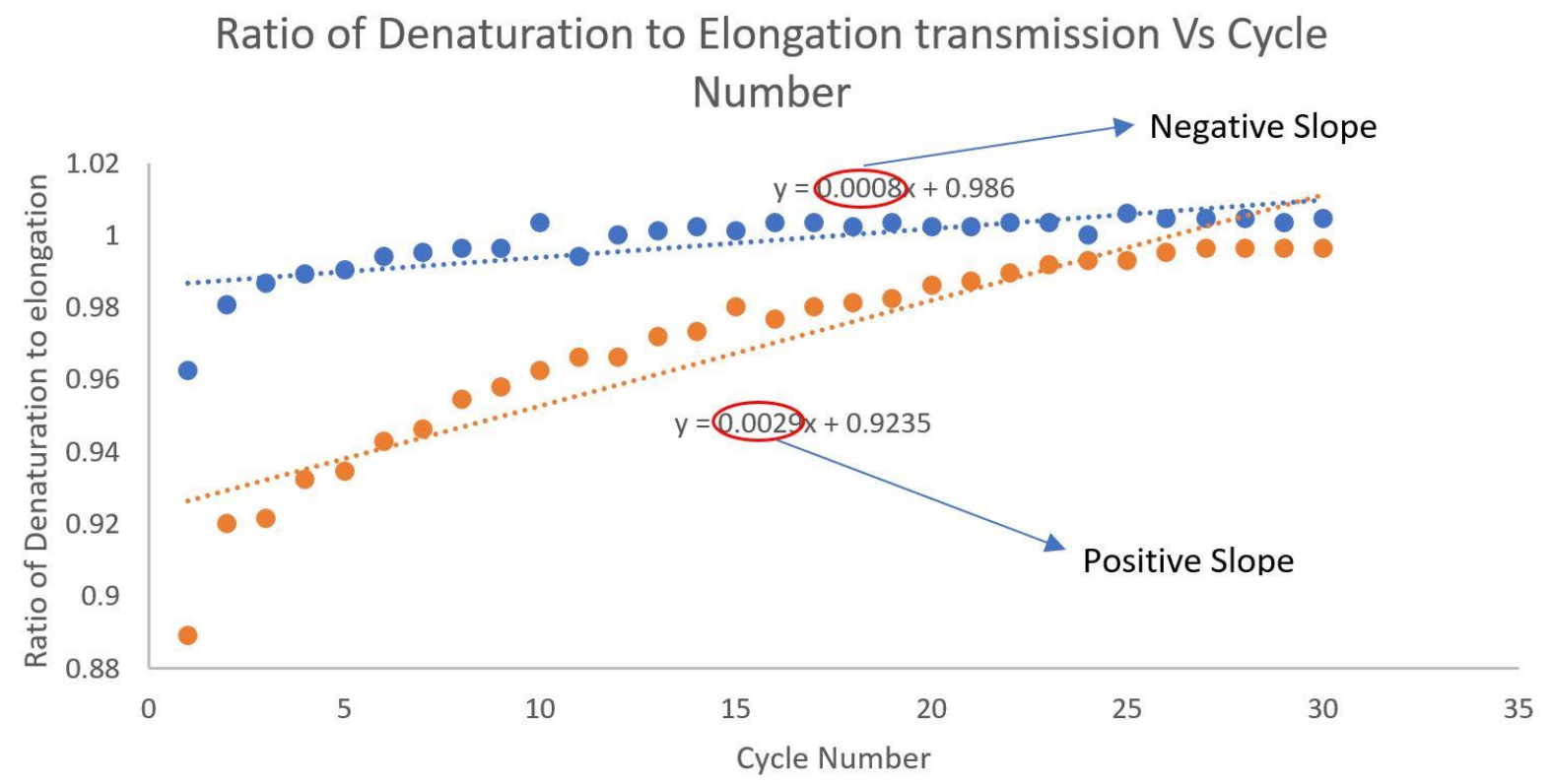

- $\quad$ Negative PCR Positive PCR $\quad \cdots \cdots . .$. Linear (Negative PCR) $\quad \cdots . . . .$. Linear (Positive PCR)

Figure 7.5: Plot of the ratio of Denaturation to Elongation Transmission Vs Cycle Number showing the Slopes of the Positive and Negative PCR Curves 


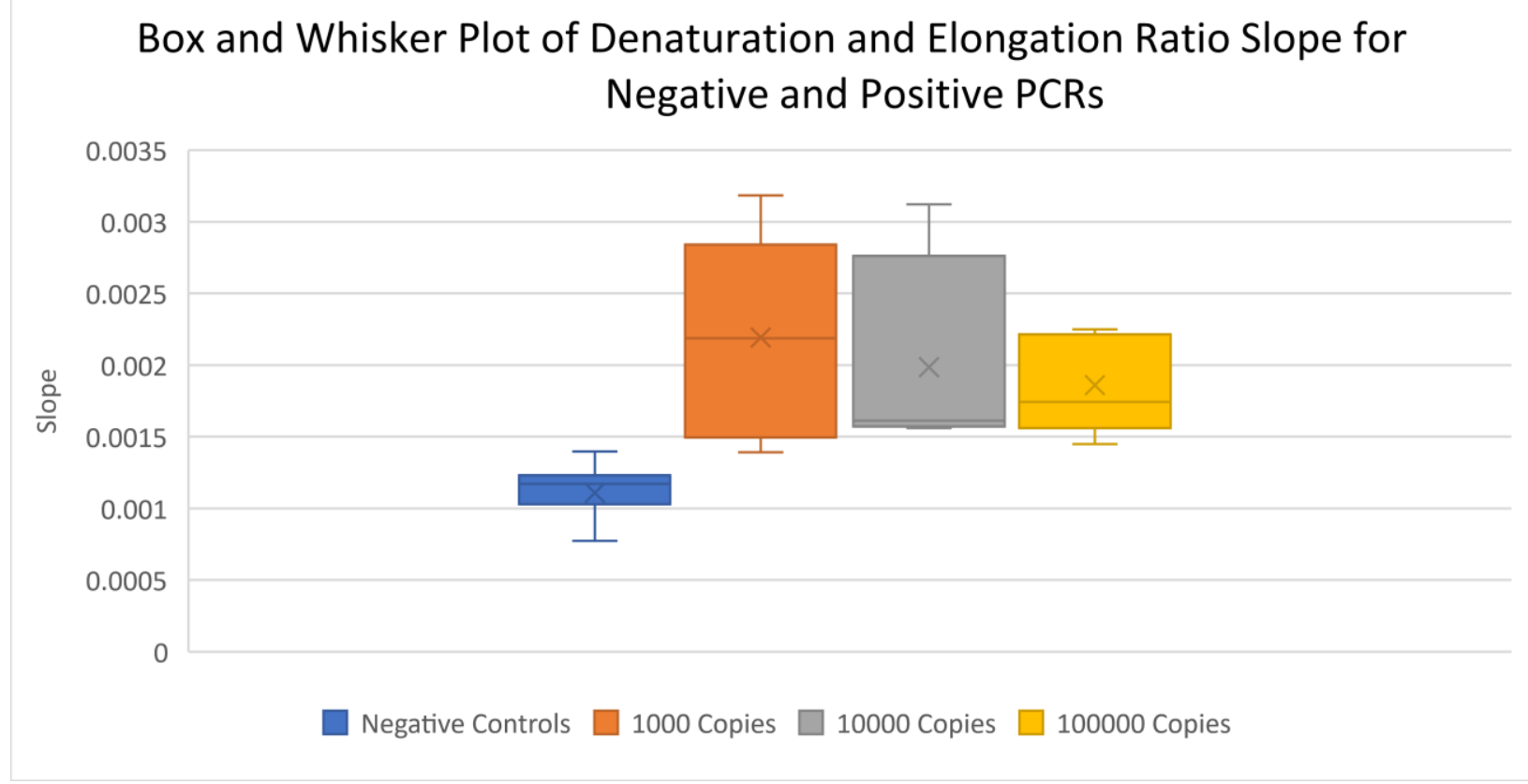

Figure 7.6: Box and Whisker Plot of Denaturation and Elongation Ratio for Negative Controls and Varying concentrations of DNA

\subsection{Phase shift Monitoring: Time Difference between Temperature and \\ Transmission Curves}

Analysis of the temperature and transmission curves of the different PCRs was carried out to determine the difference between positive and negative PCRs. This was done by considering the difference in time (simply referred to as 'phase shift' for convenience) between the maximum of the fitted temperature curve and the minimum of the fitted transmission curves. As an example, figures $7.7-7.10$ show sections of the temperature and transmission curves of cycles 10 and 30 for positive and negative PCRs used in the analysis. Using Matlab (see Appendix A), a polynomial curve fit was done for each temperature and transmission data. The time taken to attain maximum temperature and minimum transmission were extracted from the data. The difference between these times was recorded for different cycle number. Figures 7.11 shows the result of the Matlab 
code (see Appendix B) used to plot the difference in time (phase shift) between the maximum of the fitted temperature curve and the minimum of the transmission curve versus the cycle number for each PCR experiment. From the graph, there is a profound difference between the positive and negative PCRs between cycles 10 and 20.
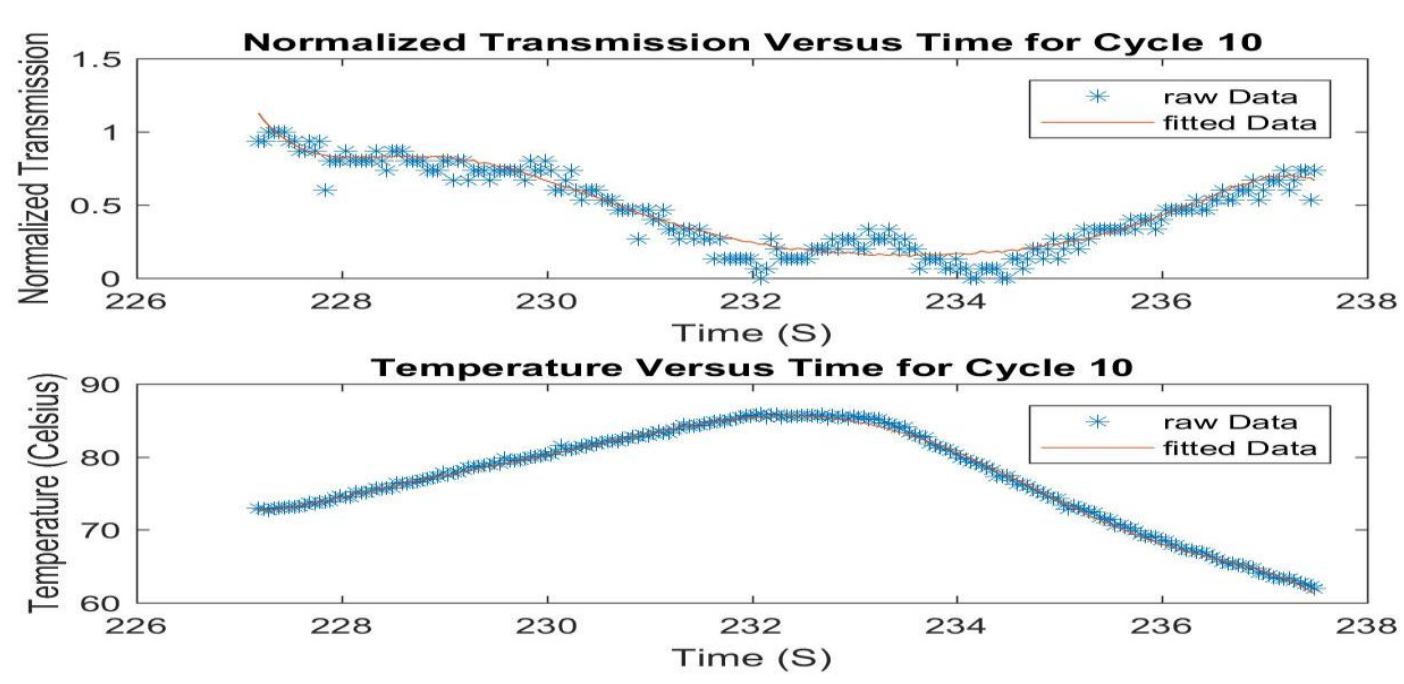

Figure 7.7. Transmission and Temperature Curves for Cycle 10 of a Negative PCR 

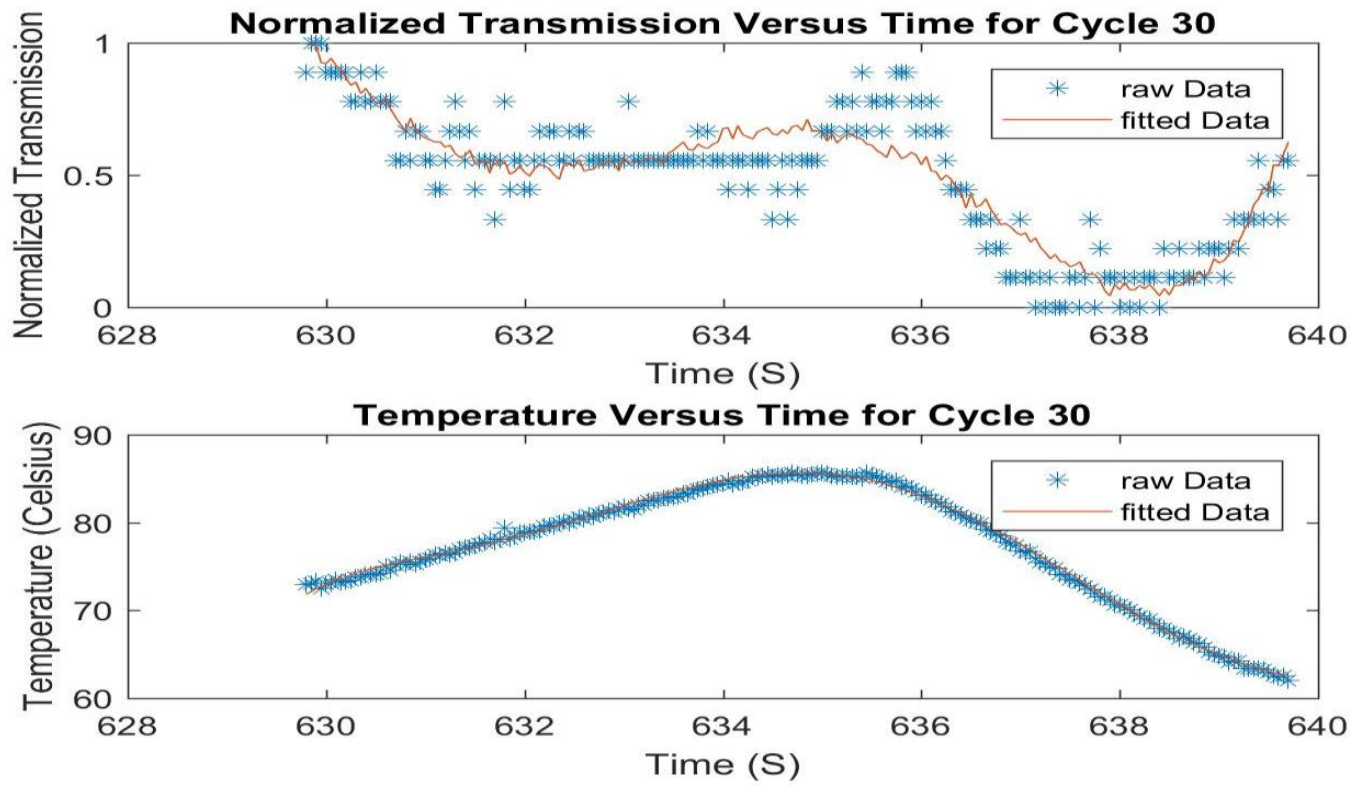

Figure 7.8: Transmission and Temperature Curves for Cycle 30 of a Negative PCR
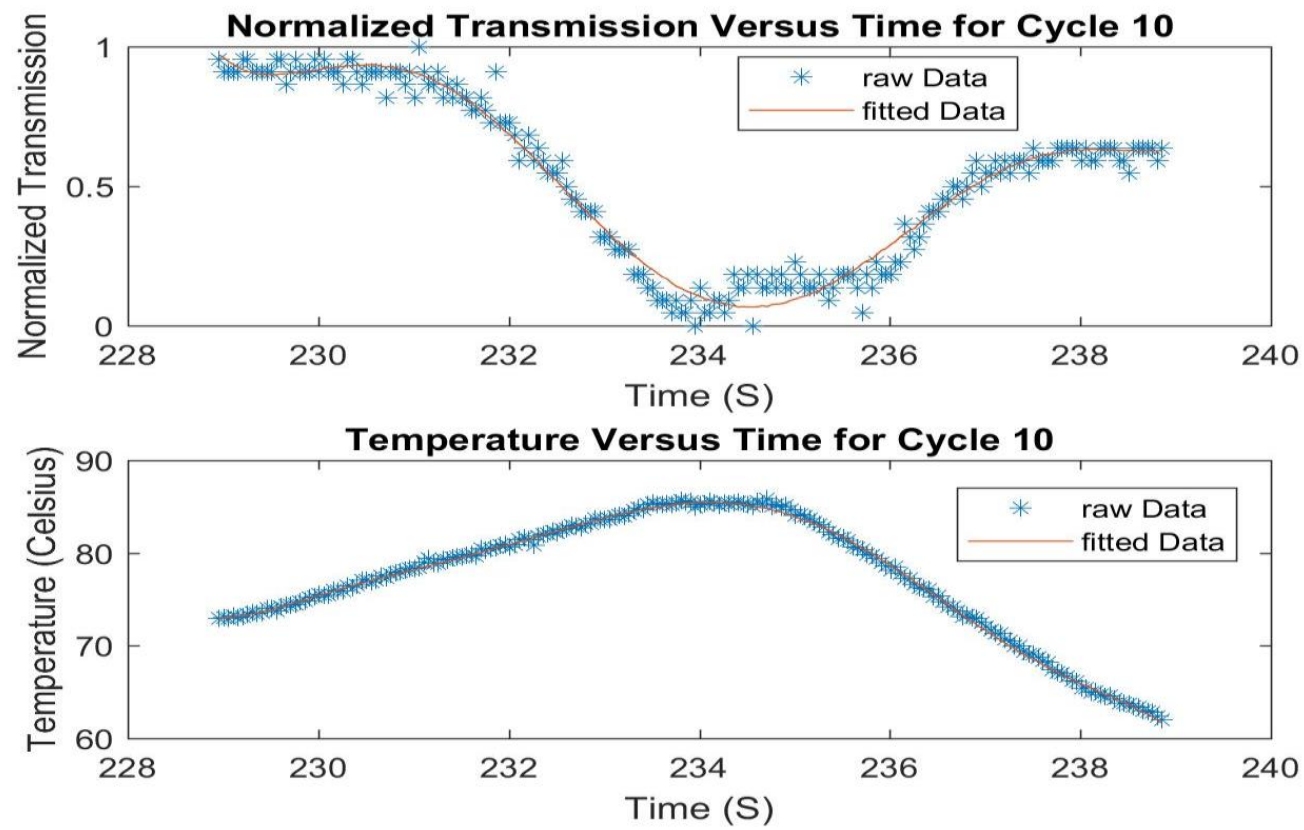

Figure 7.9: Transmission and Temperature Curves for Cycle 10 of a Positive PCR 

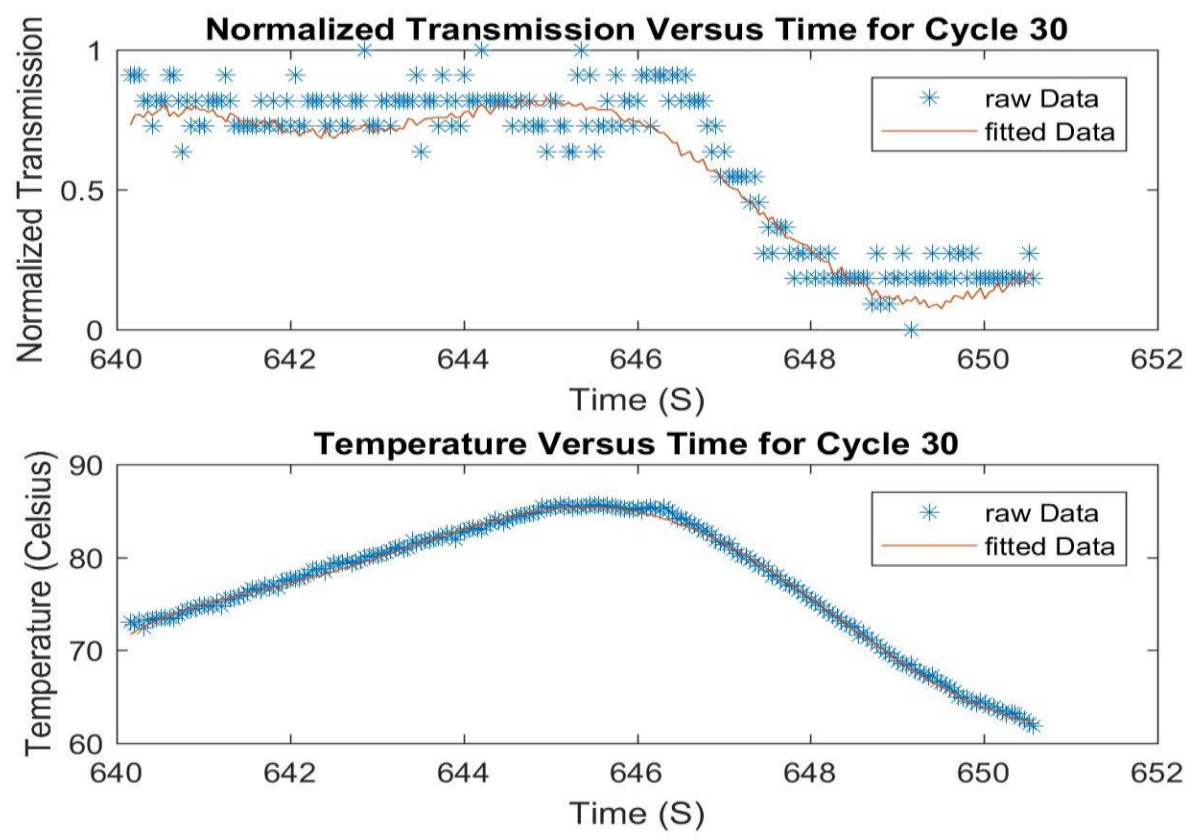

Figure 7.10: Transmission and Temperature Curves for Cycle 30 of a Positive PCR 


\section{Graph of Phase Shift Versus Cycle Number for Positive and Negative PCRs}

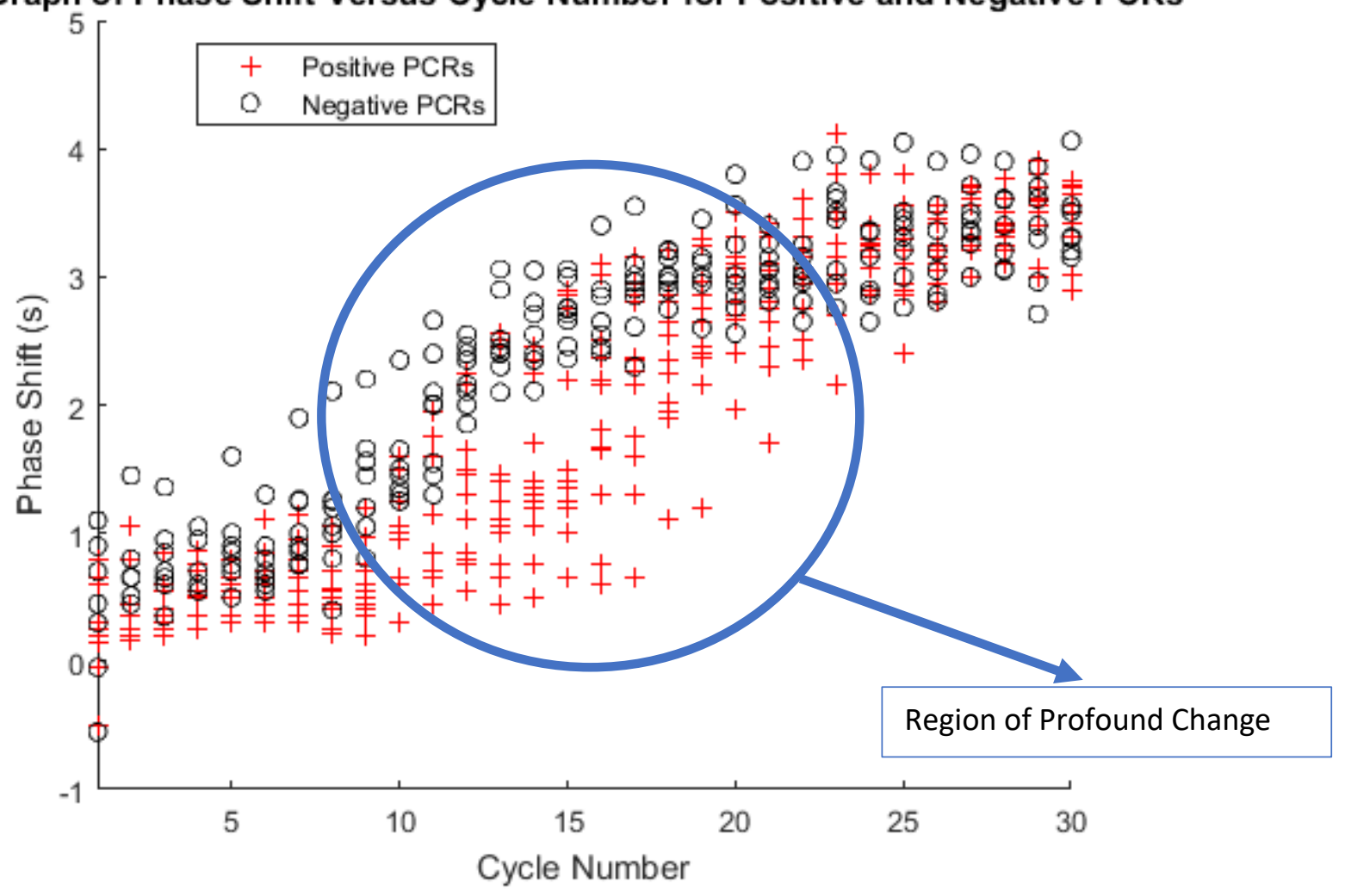

Figure 7.11: Time phase shift versus the cycle number for Positive and Negative PCRs

\subsubsection{Phase Shift Logistic Function Modelling}

To further investigate if there is a clearer trend between negative PCRs and positive PCRs of varying concentrations, we modeled the phase shift data in section 7.3 using logistic function. Although logistic function was primarily developed to model population growth, it can be extended to model S-shaped curves for which the graph of phase shift versus cycle number depicts as show in figure 7.12. A logistic curve is an " $\mathrm{S}$ " shaped curve and it is also known as a sigmoid curve. It is commonly represented by the following equation: 


$$
f(t)=\frac{L}{1+e^{-\alpha\left(t-t_{0}\right)}}
$$

where $t_{0}$ is the symmetric inflection point or the t-value of the sigmoid's midpoint, $L$ is the curve's maximum value or saturation point or the value as tends to infinity, and $\alpha$ is the time decay constant or the steepness of the curve. For different PCR experiments, we extracted the phase shift data using the Matlab code in Appendix B and did a logistic curve fit using Mathematica (see Appendix C). Figure 7.12 shows the result of the curve fit from our Mathematica code. From the results of the logistic curve fits, we then extracted the two important parameters: the symmetric inflection point and the time decay constant. These parameters were compared to each other using a simple plot to determine any difference between negative and positive PCRs. As shown in figure 7.13, the minimum point of inflection for negative PCR is around -5.5. We have determined by experiments that the maximum decay constant is between 0.7 and 0.8 ; a line drawn at 30 degrees from the point of intersection between the minimum point of inflection and half the maximum decay constant gives the dividing line between positive and negative PCRs. Also, this line helps to distinguish between positive PCRs of varying starting DNA concentrations. For example, positive PCRs are below the dividing line while the negative PCRs are above the line. For the positive PCRs, the higher concentration of DNA tends to be further from the dividing line while the lower concentrations are closer to the dividing line. Although the result shows a poor discrimination for 1000 starting copy number, our system is able to discriminate between positive PCRs with DNA starting concentration of 10,000 copy number and greater. This is comparable to most commercial PCR systems which requires more than $10^{\wedge} 4$ starting copy number. 
100,000
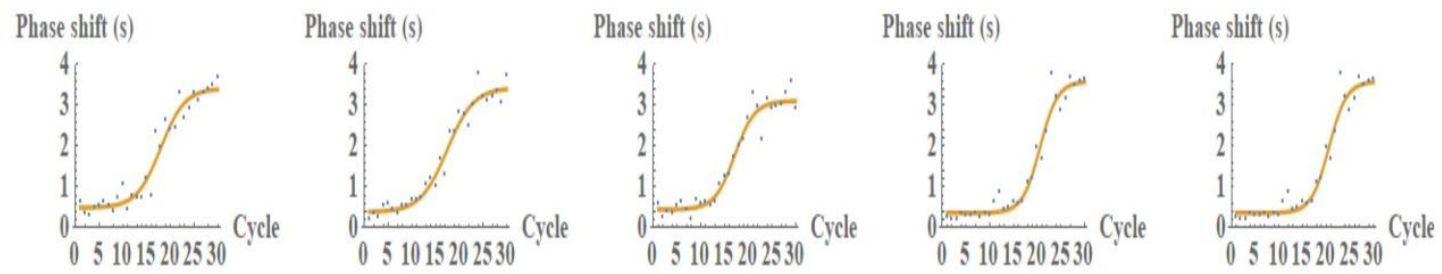

10,000
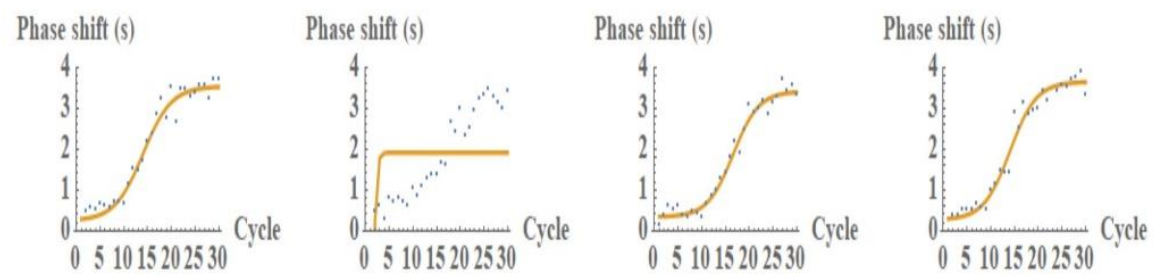

1000
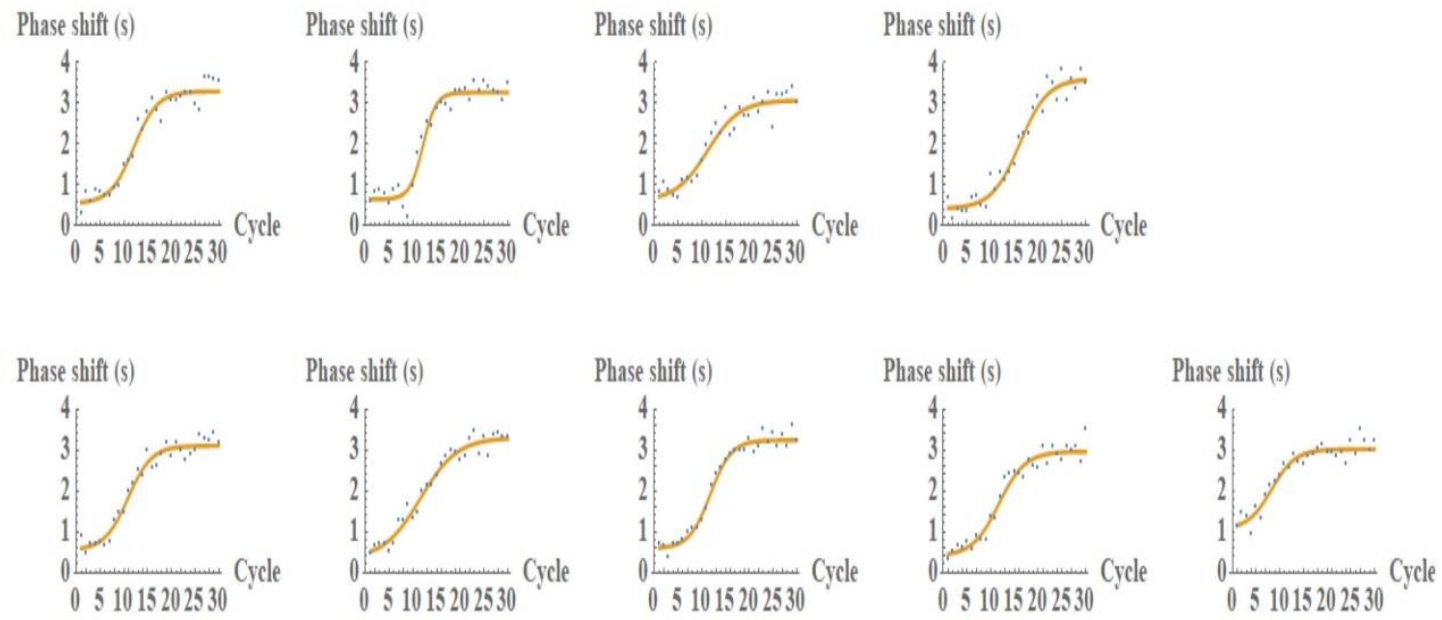

Figure 7.12: Logistic curve fits and original phase shift data plot of different PCR experiments.

The vertical axis is the normalized transmission while the horizontal axis is the Cycle Number 


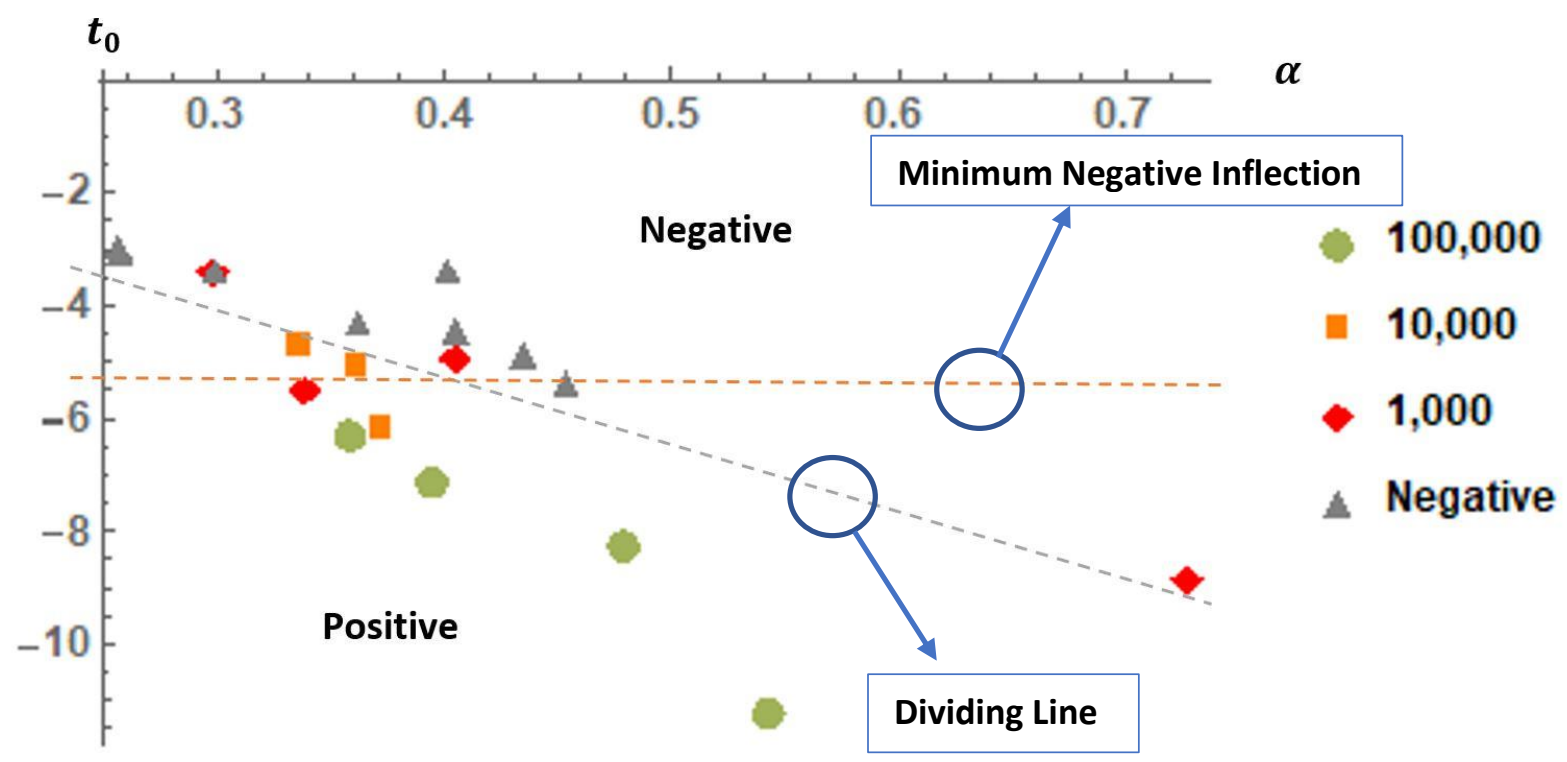

Figure 7.13: Plot of Symmetric Inflection point $\left(t_{0}\right)$ against Decay Constant $(\alpha)$

Having explored amplitude monitoring and phase monitoring in this chapter, the results obtained show that phase monitoring of DNA amplification using our plasmonic system has the potential to achieve lower limit of detection and higher throughput compared to the amplitude monitoring. 


\section{Chapter 8: Conclusion}

In this work we have demonstrated a label-free LSPR plasmonic PCR system that is capable of detecting DNA as low as 10,000 genome copies using phase shift monitoring. This is comparable to many conventional PCR systems with similar limit of detection and offers better advantages in terms of cost, speed and high throughput. Our system permits continuous monitoring of PCR reaction unlike label-based PCR systems that only provide endpoint readout. The risks associated with the use of isotope-labels are eliminated in our system since no label is required. Also, the amount of labor required in sample preparation common with most label-based conventional PCR is reduced making the process less complicated.

Our PCR system was successfully adapted to run loop-mediated isothermal amplification (LAMP). It has the potential to carryout rapid PCR and quantify amplicon production in real time. It also utilizes standard size PCR tubes and the components used in the optical design do not require the usual complicated process of fabrication associated with most convention PCR systems. It is also easy to operate hence, our system can be effectively utilized as a POC diagnostic device. As such our plasmonic PCR system can be easily deployed in developing countries to help combat the spread of diseases such as Ebola. It can also be used for forensic purposes.

\subsection{Future Work}

The plasmonic PCR system described in this work is a one channel system, which means only one PCR reaction can be carried out at a time thereby increasing the time it takes to run several PCR reactions. This could be a major limitation in clinical application where several samples of PCR are required. This can be improved upon by incorporating a multi-channel heating system by splitting a high-power laser beam into lower power beamlets for thermocycling. Also, the 808nm 
edge emitting semiconductor laser used in this work for heating is bulky and therefore makes it difficult to design a miniaturized system; another type of semiconductor lasers diodes such as the vertical-cavity surface-emitting laser(VCSEL) can be used as alternative for more efficient heating and precision in sensing.

The detection or monitoring system in this work can be improved upon by designing the compartment of the system in such a way that it is less prone to optical background noise. A better cage system would be useful for this purpose. Also, experiments carried out on the system show that the heating laser contributes a significant amount of noise to the system and as such interferes with the output from the probe laser. Although, we overcame this challenge by taking the lock-in reading only when the laser and fan were turned off, there was a significant increase in the time taken for a PCR reaction to be completed. Good optical filtering could be employed in future work to solve this problem.

An alternative sensing method involving the use of another nanoparticle as optical sensor can be employed in future work. This would help eliminate errors arising from the shift in resonance of the nanoparticle used for heating. 


\section{Appendix A}

\section{Matlab function slope to extract data from the region of interest (from ramp up to cooling)}

clc;

clear all;

filename $=$ 'Negative Controls Matlab Analysis';

sheet $=1$;

$\mathrm{M}=$ xlsread(filename, sheet);

cycle $=\mathrm{M}(:, 1)$

time $=\mathrm{M}(:, 2)$;

temp $=\mathrm{M}(:, 3)$;

trans $=\mathrm{M}(:, 4)$;

NumberofCycles $=30$;

holdTime $=0.0$;

Dmin $=80.01 ; \%$ Denaturation Minimum limit

Dmax $=100.05 ; \%$ Denaturation Maximum limit

Amin $=57.01 ; \%$ Annealing Minimum limit

Amax $=62.05 ; \%$ Annealing Maximum limit

Emin $=71.01 ; \%$ Annealing Minimum limit

Emax $=73.05 ; \%$ Annealing Maximum limit

indexcycle $=\{\}$;

for $\mathrm{k}=1$ :max (cycle)

indexcycle $\{\mathrm{k}\}=$ find $($ cycle $==\mathrm{k})$;

end

for $\mathrm{y}=1$ :length(indexcycle)

for $x=1$ : length(indexcycle $\{y\})$

newCycle $\{\mathrm{y}\}(\mathrm{x})=\operatorname{cycle}($ indexcycle $\{\mathrm{y}\}(\mathrm{x}))$;

newTime $\{y\}(x)=\operatorname{time}($ indexcycle $\{y\}(x))$;

newTemp $\{y\}(x)=$ temp $($ indexcycle $\{y\}(x))$;

newTrans $\{y\}(x)=\operatorname{trans}($ indexcycle $\{y\}(x))$;

end

end

for $\mathrm{j}=1$ :length(indexcycle)

$\% \%$ Extracting Denaturation to Elongation Data 
indexDtemp $\{\mathrm{j}\}=$ find $($ newTemp $\{\mathrm{j}\}>\operatorname{Dmin} \&$ newTemp $\{\mathrm{j}\}<\operatorname{Dmax})$;

firstIndex $(\mathrm{j})=$ indexDtemp $\{\mathrm{j}\}(1) ; \%$ get the first element of the indexDtemp when denaturation started

lastIndex $(\mathrm{j})=$ length $($ newTemp $\{\mathrm{j}\}) ; \%$ End of newTemp or data point of temperature

newIndexDtemp $\{\mathrm{j}\}=($ firstIndex $(\mathrm{j})): 1: \operatorname{lastIndex}(\mathrm{j}) ; \%$ New index of temperature beginning from the denaturation temp.

rampDIndex $\{j\}=1: 1:($ firstIndex $(j)) ; \%$ Heating to denaturation phase

for $\mathrm{x}=1$ : length(newIndexDtemp $\{\mathrm{j}\}) \%$ Extracting data from denaturation stage to elongation stage

$\mathrm{dToeCycle}\{\mathrm{j}\}(\mathrm{x})=$ newCycle $\{\mathrm{j}\}($ newIndexDtemp $\{\mathrm{j}\}(\mathrm{x}))$;

$\operatorname{dToeTime}\{\mathrm{j}\}(\mathrm{x})=$ newTime $\{\mathrm{j}\}($ newIndexDtemp $\{\mathrm{j}\}(\mathrm{x}))$;

$\mathrm{dToeTemp}\{\mathrm{j}\}(\mathrm{x})=\operatorname{newTemp}\{\mathrm{j}\}($ newIndexDtemp $\{\mathrm{j}\}(\mathrm{x}))$;

$\operatorname{dToeTrans}\{\mathrm{j}\}(\mathrm{x})=\operatorname{new} \operatorname{Trans}\{\mathrm{j}\}(\operatorname{newIndexDtemp}\{\mathrm{j}\}(\mathrm{x}))$;

end

for $\mathrm{x}=1$ : length(rampDIndex $\{\mathrm{j}\})$

$\%$ Heating to Denaturation phase

$\operatorname{rampCycle}\{\mathrm{j}\}(\mathrm{x})=$ newCycle $\{\mathrm{j}\}(\operatorname{rampDIndex}\{\mathrm{j}\}(\mathrm{x}))$;

rampTime $\{\mathrm{j}\}(\mathrm{x})=$ newTime $\{\mathrm{j}\}(\operatorname{rampDIndex}\{\mathrm{j}\}(\mathrm{x}))$;

$\operatorname{rampTemp}\{\mathrm{j}\}(\mathrm{x})=$ newTemp $\{\mathrm{j}\}(\operatorname{rampDIndex}\{\mathrm{j}\}(\mathrm{x}))$;

$\operatorname{rampTrans}\{\mathrm{j}\}(\mathrm{x})=$ newTrans $\{\mathrm{j}\}(\operatorname{rampDIndex}\{\mathrm{j}\}(\mathrm{x}))$;

end

$\% \%$ Extracting Annealing to Elongation Data

indexAtemp $\{\mathrm{j}\}=$ find $(\mathrm{dToeTemp}\{\mathrm{j}\}>\operatorname{Amin} \& \operatorname{dToeTemp}\{\mathrm{j}\}<\operatorname{Amax}) ; \%$ Get annealing

Temperature

firstAIndex $(j)=\operatorname{indexAtemp}\{j\}(1) ; \%$ get first measure of annealing temperature

lastAIndex $(\mathrm{j})=$ length $(\operatorname{dToeTemp}\{\mathrm{j}\}) ; \%$ temperature up measure to elongation

newIndexAtemp $\{\mathrm{j}\}=($ firstAIndex $(\mathrm{j})): 1:$ lastAIndex $(\mathrm{j}) ; \%$ Index from annealing to elongation

coolIndex $\{\mathrm{j}\}=1: 1$ :(firstAIndex $(\mathrm{j})) ; \%$ cooling from denaturation to annealing phase

for $\mathrm{x}=1$ : length(coolIndex $\{\mathrm{j}\})$

$\%$ Heating to Denaturation phase

$\operatorname{coolCycle}\{\mathrm{j}\}(\mathrm{x})=\mathrm{dToeCycle}\{\mathrm{j}\}(\operatorname{coolIndex}\{\mathrm{j}\}(\mathrm{x}))$;

coolTime $\{\mathrm{j}\}(\mathrm{x})=\mathrm{dToeTime}\{\mathrm{j}\}(\operatorname{coolIndex}\{\mathrm{j}\}(\mathrm{x}))$;

$\operatorname{coolTemp}\{\mathrm{j}\}(\mathrm{x})=\mathrm{dToeTemp}\{\mathrm{j}\}(\operatorname{coolIndex}\{\mathrm{j}\}(\mathrm{x}))$;

coolTrans $\{\mathrm{j}\}(\mathrm{x})=\mathrm{dToeTrans}\{\mathrm{j}\}(\operatorname{coolIndex}\{\mathrm{j}\}(\mathrm{x}))$; 
end

end

$\% \%$ Extracting Data from heating to denaturation to cooling to annealing

for $\mathrm{x}=1: 30$

$\operatorname{trans} 1=\operatorname{ramp} \operatorname{Trans}\{\mathrm{x}\}$;

trans $2=\operatorname{coolTrans}\{\mathrm{x}\}$;

$\operatorname{aedTrans}\{x\}=\operatorname{horzcat}(\operatorname{trans} 1, \operatorname{trans} 2)$;

normAEDTrans $\{x\}=(\operatorname{aedTrans}\{x\}-\min (\operatorname{aedTrans}\{x\})) . / \operatorname{range}(\operatorname{aedTrans}\{x\})$;

time2=coolTime $\{x\}$;

time1=rampTime $\{\mathrm{x}\}$;

aedTime $\{\mathrm{x}\}=\operatorname{horzcat}($ time1,time2);

temp2=coolTemp $\{\mathrm{x}\}$

temp1=rampTemp $\{\mathrm{x}\}$;

aedTemp $\{x\}=\operatorname{horzcat}($ temp1,temp2);

end

for $\mathrm{i}=1: 30$

$[\mathrm{p}, \mathrm{S}]=\operatorname{polyfit}(\operatorname{aedTime}\{\mathrm{i}\}$, normAEDTrans $\{\mathrm{i}\}, 10)$;

$\mathrm{f}\{\mathrm{i}\}=\operatorname{polyval}(\mathrm{p}, \operatorname{aedTime}\{\mathrm{i}\})$;

$[\mathrm{m}$, Indices $]=\min (\mathrm{f}\{\mathrm{i}\})$;

minTime $=$ aedTime $\{\mathrm{i}\}$ (Indices);

title(['Order is: ',num2str(10),' norm is: ',num2str(S.normr)])

[p2,S2] = polyfit (aedTime $\{\mathrm{i}\}, \operatorname{aedTemp}\{\mathrm{i}\}, 10)$;

$\mathrm{f} 2\{\mathrm{i}\}=\operatorname{polyval}(\mathrm{p} 2, \operatorname{aedTime}\{\mathrm{i}\})$;

$[\mathrm{m} 2$, Indices 2$]=\max (\mathrm{f} 2\{\mathrm{i}\})$;

maxTime $2=\operatorname{aedTime}\{\mathrm{i}\}($ Indices 2$)$;

title(['Order is: ',num2str(10),' norm is: ',num2str(S.normr)])

phi $(\mathrm{i})=\min$ Time $-\max$ Time 2 ;

end

$\operatorname{ax} 1=\operatorname{subplot}(2,1,1)$ 
plot(ax 1, aedTime $\{30\}$, normAEDTrans $\{30\},{ }^{\prime} *$ ',aedTime $\left.\{30\}, f\{30\},{ }^{-}-'\right)$

title('Normalized Transmission Versus Time for Cycle 30');

legend('raw Data', 'fitted Data');

xlabel('Time $\left.(\mathrm{S})^{\prime}\right)$;

ylabel('Normalized Transmission');

ax2 = subplot $(2,1,2)$;

plot(ax2, aedTime $\{30\}$, aedTemp $\{30\}$,'*', aedTime $\left.\{30\}, f 2\{30\},,^{-}-'\right)$

title('Temperature Versus Time for Cycle 30')

legend('raw Data', 'fitted Data');

xlabel('Time $(\mathrm{S})$ ');

ylabel('Temperature (Celsius)'); 


\section{Appendix B}

\section{Matlab Code for Phase shift Versus Cycle Number}

clc;

clear all;

excelFilename $=$ 'PhaseShift_Raw_Data_2018_06_28';

sheet $=21$;

for $i=1$ :sheet

phi $(:, \mathrm{i})=$ phaseShift $($ excelFilename, $\mathrm{i})$;

end

cycle $=$ transpose $([1: 1: 30])$;

slopeTrans $=$ [cycle, phi];

[rows columns] = size( slopeTrans);

for $\mathrm{i}=2$ : columns

$y \lim \left(\left[\begin{array}{ll}-1 & 5])\end{array}\right.\right.$

$\mathrm{x} \lim \left(\left[\begin{array}{ll}1 & 30])\end{array}\right.\right.$

if $\mathrm{i}<=14$

h1=scatter(slopeTrans(:,1), slopeTrans(:,i), 'r', '+');

hold all;

else

h2=scatter( $\left.\operatorname{slopeTrans}(:, 1), \operatorname{slopeTrans}(:, \mathrm{i}), \mathrm{k}^{\prime}, \mathrm{o}^{\prime}\right)$;

hold all;

end

end

legend([h1 h2], \{'Positive PCRs','Negative PCRs' $\})$

xlabel('Cycle Number');

ylabel('Phase Shift (s)');

title('Graph of Phase Shift Versus Cycle Number for Positive and Negative PCRs'); 


\section{Appendix C}

Mathematica Code for Logistic Curve Fit

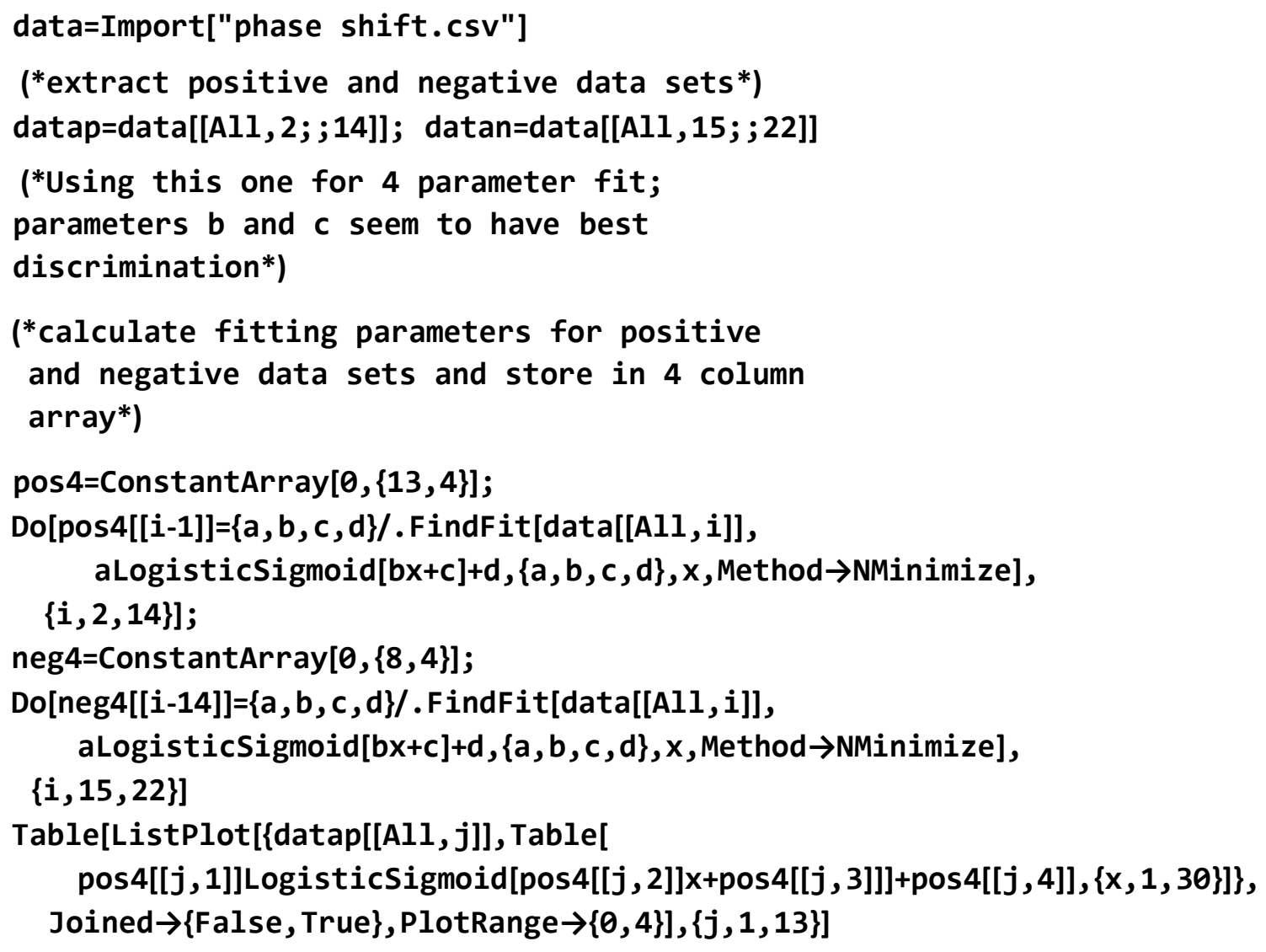

Table[ListPlot [\{datan[[All, j]], Table[ neg4[[j, 1]]LogisticSigmoid[neg4[[j, 2]]x+neg4[[j, 3]]]+neg4[[j, 4]], $\{x, 1,30\}]\}$, Joined $\rightarrow\{$ False, True $\}$, PlotRange $\rightarrow\{0,4\}],\{j, 1,8\}]$

TableForm[pos4]

$\begin{array}{llll}2.92583 & 0.395101 & -7.0981 & 0.493037 \\ 3.04304 & 0.358982 & -6.25649 & 0.388838 \\ 2.66334 & 0.479434 & -8.21819 & 0.44915 \\ 3.22426 & 0.543575 & -11.191 & 0.365119 \\ 3.22426 & 0.543575 & -11.191 & 0.365119 \\ 3.29356 & 0.336181 & -4.71811 & 0.241461 \\ -1602.18 & -2.58866 & -1.68775 & 1.91164\end{array}$




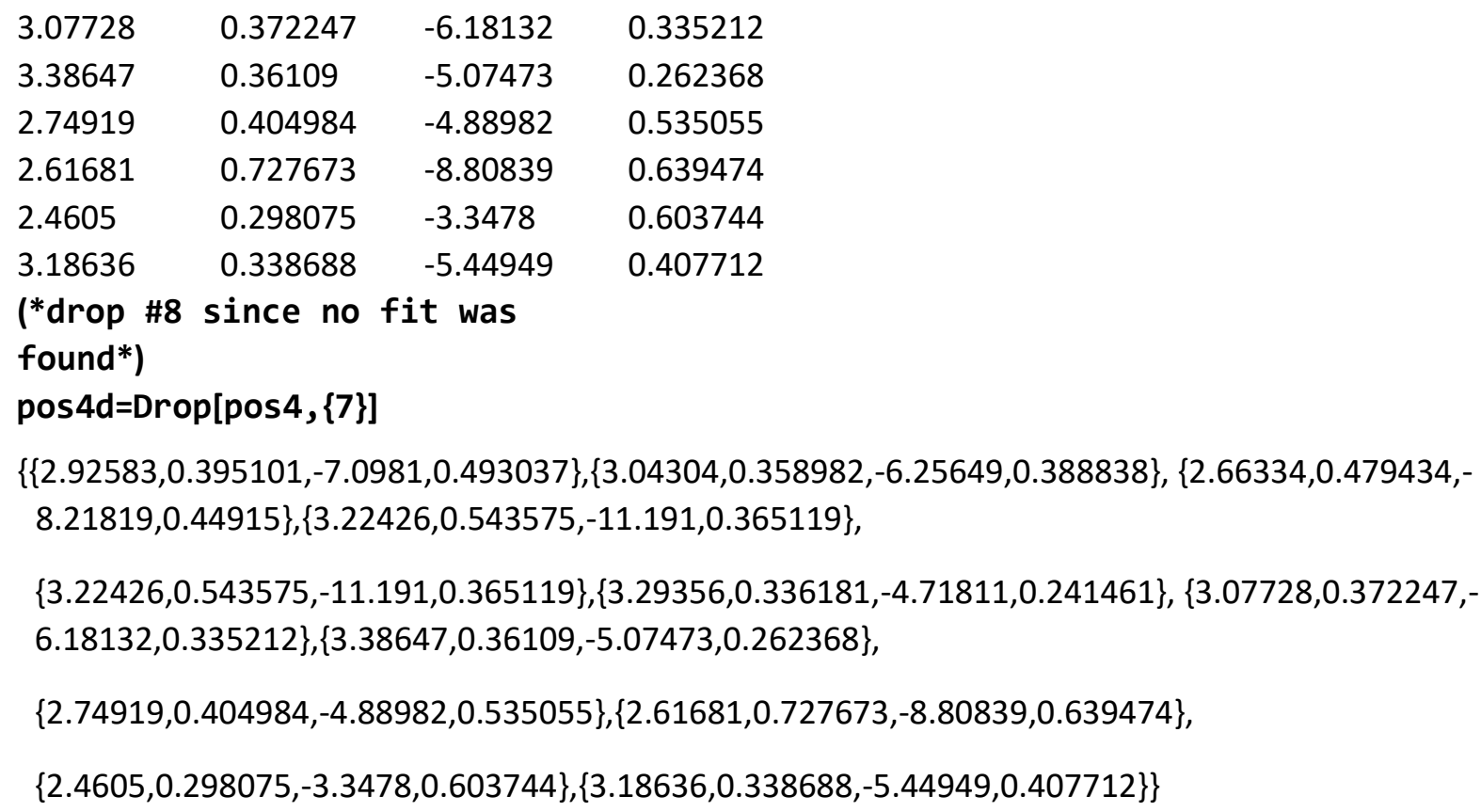

$2.46050 .298075-3.34780 .6037443 .186360 .338688$ -

5.449490 .407712

TableForm[neg4]

$\begin{array}{llll}2.55338 & 0.40529 & -4.4082 & 0.542152 \\ 2.97907 & 0.257169 & -2.97887 & 0.316223 \\ 2.65415 & 0.453973 & -5.3458 & 0.579533 \\ 2.55944 & 0.362402 & -4.23792 & 0.394001\end{array}$




\section{References}

[1] J. Singh, a critical review on PCR, its types and applications. 2014, pp. 65-80.

[2] K. B. Mullis and F. A. Faloona, "[21] Specific synthesis of DNA in vitro via a polymerase-catalyzed chain reaction," in Methods in Enzymology, vol. 155: Academic Press, 1987, pp. 335-350.

[3] R. K. Saiki et al., "Primer-directed enzymatic amplification of DNA with a thermostable DNA polymerase," Science, 10.1126/science.239.4839.487 vol. 239, no. 4839, p. 487, 1988.

[4] J. M. Bartlett and D. Stirling, "A Short History of the Polymerase Chain Reaction," in PCR Protocols, vol. 226(Methods in Molecular Biology, 2003, pp. 3-6.

[5] BioNinja. (2018, April 4). Overview of a Polymerase Chain Reaction Cycle. Available: http://ib.bioninja.com.au/standard-level/topic-3-genetics/35-genetic-modification-and/pcr.html

[6] M. T. Rahman, M. S. Uddin, R. Sultana, A. Moue, and M. Setu, "Polymerase Chain Reaction (PCR): A Short Review," 2013, PCR; Molecular techniques; Review vol. 4, no. 1, p. 7, 2013-02-06 2013.

[7] F. J. Jenkins, "Basic methods for the detection of PCR products," (in eng), PCR Methods Appl, vol. 3, no. 5, pp. S77-82, Apr 1994.

[8] J. G. Lazar, "Advanced methods in PCR product detection," (in eng), PCR Methods Appl, vol. 4, no. 1, pp. S1-14, Aug 1994.

[9] R. Higuchi, G. Dollinger, P. S. Walsh, and R. Griffith, "Simultaneous Amplification and Detection of Specific DNA Sequences," Bio/Technology, vol. 10, p. 413, 04/01/online 1992.

[10] C. Gachon, A. Mingam, and B. Charrier, "Real-time PCR: what relevance to plant studies?," Journal of Experimental Botany, vol. 55, no. 402, pp. 1445-1454, 2004.

[11] S. A. Deepak et al., "Real-Time PCR: Revolutionizing Detection and Expression Analysis of Genes," Current Genomics, vol. 8, no. 4, pp. 234-251, 01/10/received 02/27/revised 03/02/accepted 2007.

[12] K. V, DNA Biosensors-A Review. 2017.

[13] V. Crivianu-Gaita and M. Thompson, "Aptamers, antibody scFv, and antibody Fab' fragments: An overview and comparison of three of the most versatile biosensor biorecognition elements," Biosensors and Bioelectronics, vol. 85, pp. 32-45, 2016/11/15/ 2016.

[14] C. I. L. Justino, A. C. Freitas, R. Pereira, A. C. Duarte, and T. A. P. Rocha Santos, "Recent developments in recognition elements for chemical sensors and biosensors," TrAC - Trends in Analytical Chemistry, Review vol. 68, pp. 2-17, 2015.

[15] F. C. Sandrine, "Surface Plasmon Resonance Biosensor for the Measurement of Complex Refractive Indices and the Monitoring of Bacterial Cell Growth," PhD ECE, McGill University, Canada, 2016.

[16] H. S. Jang, K. N. Park, C. D. Kang, J. P. Kim, S. J. Sim, and K. S. Lee, "Optical fiber SPR biosensor with sandwich assay for the detection of prostate specific antigen," Optics Communications, vol. 282, no. 14, pp. 2827-2830, 2009/07/15/ 2009.

[17] L. Zhang, R. Huang, W. Liu, H. Liu, X. Zhou, and D. Xing, "Rapid and visual detection of Listeria monocytogenes based on nanoparticle cluster catalyzed signal amplification," Biosensors and Bioelectronics, vol. 86, pp. 1-7, 2016/12/15/ 2016.

[18] R. Irawan, Y. H. Cheng, W. M. Ng, M. M. Aung, I. K. Lao, and V. Thaveeprungsriporn, "Polymer waveguide sensor for early diagnostic and wellness monitoring," Biosensors and Bioelectronics, vol. 26, no. 8, pp. 3666-3669, 2011/04/15/ 2011.

[19] R. Bruck, E. Melnik, P. Muellner, R. Hainberger, and M. Lämmerhofer, "Integrated polymer-based Mach-Zehnder interferometer label-free streptavidin biosensor compatible with injection molding," Biosensors and Bioelectronics, vol. 26, no. 9, pp. 3832-3837, 2011/05/15/ 2011. 
[20] C. Wu, Y. Zhou, X. Miao, and L. Ling, "A novel fluorescent biosensor for sequence-specific recognition of double-stranded DNA with the platform of graphene oxide," Analyst, 10.1039/C1AN15061H vol. 136, no. 10, pp. 2106-2110, 2011.

[21] J.-j. Li, H.-q. An, J. Zhu, and J.-w. Zhao, "Detecting glucose by using the Raman scattering of oxidized ascorbic acid: The effect of graphene oxide-gold nanorod hybrid," Sensors and Actuators B: Chemical, vol. 235, pp. 663-669, 2016/11/01/ 2016.

[22] J. H. Wade and R. C. Bailey, "Applications of Optical Microcavity Resonators in Analytical Chemistry," Annual Review of Analytical Chemistry, vol. 9, no. 1, pp. 1-25, 2016/06/12 2016.

[23] P. Schubert-Ullrich et al., "Commercialized rapid immunoanalytical tests for determination of allergenic food proteins: an overview," Analytical and Bioanalytical Chemistry, vol. 395, no. 1, pp. 69-81, 2009/09/01 2009.

[24] L. Monaci, M. Brohée, V. Tregoat, and A. van Hengel, "Influence of baking time and matrix effects on the detection of milk allergens in cookie model food system by ELISA," Food Chemistry, vol. 127, no. 2, pp. 669-675, 2011/07/15/ 2011.

[25] H. Berney, J. West, E. Haefele, J. Alderman, W. Lane, and J. K. Collins, "A DNA diagnostic biosensor: development, characterisation and performance," Sensors and Actuators B: Chemical, vol. 68, no. 1, pp. 100-108, 2000/08/25/ 2000.

[26] J. Juan Colas, Dual-Mode Electro-photonic Silicon Biosensors (Recognizing Outstanding Ph.D. Research). Springer, 2017.

[27] A. Syahir, K. Usui, K.-y. Tomizaki, K. Kajikawa, and H. Mihara, "Label and Label-Free Detection Techniques for Protein Microarrays," Microarrays, vol. 4, no. 2, p. 228, 2015.

[28] D. M. Rissin et al., "Single-molecule enzyme-linked immunosorbent assay detects serum proteins at subfemtomolar concentrations," Nature Biotechnology, vol. 28, p. 595, 05/23/online 2010.

[29] J. E. Celis and P. Gromov, "2D protein electrophoresis: can it be perfected?," Current Opinion in Biotechnology, vol. 10, no. 1, pp. 16-21, 1999/02/01/ 1999.

[30] A. J. Wardeh et al., " $\beta$-Particle-Emitting Radioactive Stent Implantation," A Safety and Feasibility Study, vol. 100, no. 16, pp. 1684-1689, 1999.

[31] M. Eigen and R. Rigler, "Sorting single molecules: application to diagnostics and evolutionary biotechnology," Proceedings of the National Academy of Sciences, vol. 91, no. 13, pp. 5740-5747, 1994.

[32] P. Schwille, U. Haupts, S. Maiti, and W. W. Webb, "Molecular Dynamics in Living Cells Observed by Fluorescence Correlation Spectroscopy with One- and Two-Photon Excitation," Biophysical Journal, vol. 77, no. 4, pp. 2251-2265, 1999/10/01/ 1999.

[33] N. Doi et al., "Novel Fluorescence Labeling and High-Throughput Assay Technologies for In Vitro Analysis of Protein Interactions," Genome Research, vol. 12, no. 3, pp. 487-492, 10/11/received 12/14/accepted 2002.

[34] R. Sandipan, M. Gunjan, and S. Sanjeeva, "Label-free detection techniques for protein microarrays: Prospects, merits and challenges," PROTEOMICS, vol. 10, no. 4, pp. 731-748, 2010.

[35] K. M. Mayer and J. H. Hafner, "Localized Surface Plasmon Resonance Sensors," Chemical Reviews, vol. 111, no. 6, pp. 3828-3857, 2011/06/08 2011.

[36] S. Unser, I. Bruzas, J. He, and L. Sagle, "Localized Surface Plasmon Resonance Biosensing: Current Challenges and Approaches," (in eng), Sensors (Basel), vol. 15, no. 7, pp. 15684-716, Jul 22015.

[37] A. J. Haes and R. P. Van Duyne, "A Nanoscale Optical Biosensor: Sensitivity and Selectivity of an Approach Based on the Localized Surface Plasmon Resonance Spectroscopy of Triangular Silver Nanoparticles," Journal of the American Chemical Society, vol. 124, no. 35, pp. 10596-10604, 2002/09/01 2002. 
[38] J. C. Riboh, A. J. Haes, A. D. McFarland, C. Ranjit Yonzon, and R. P. Van Duyne, "A Nanoscale Optical Biosensor: Real-Time Immunoassay in Physiological Buffer Enabled by Improved Nanoparticle Adhesion," The Journal of Physical Chemistry B, vol. 107, no. 8, pp. 1772-1780, 2003/02/01 2003.

[39] C. R. Yonzon, E. Jeoung, S. Zou, G. C. Schatz, M. Mrksich, and R. P. Van Duyne, "A Comparative Analysis of Localized and Propagating Surface Plasmon Resonance Sensors: The Binding of Concanavalin A to a Monosaccharide Functionalized Self-Assembled Monolayer," Journal of the American Chemical Society, vol. 126, no. 39, pp. 12669-12676, 2004/10/01 2004.

[40] H. Anders, B. Anna, N. Olof, L. Morten, L. Joakim, and U. Mathias, "The biotin-streptavidin interaction can be reversibly broken using water at elevated temperatures," ELECTROPHORESIS, vol. 26, no. 3, pp. 501-510, 2005.

[41] S. M. Marinakos, S. Chen, and A. Chilkoti, "Plasmonic Detection of a Model Analyte in Serum by a Gold Nanorod Sensor," Analytical Chemistry, vol. 79, no. 14, pp. 5278-5283, 2007/07/01 2007.

[42] Y. Wang, W. Qian, Y. Tan, and S. Ding, "A label-free biosensor based on gold nanoshell monolayers for monitoring biomolecular interactions in diluted whole blood," Biosensors and Bioelectronics, vol. 23, no. 7, pp. 1166-1170, 2008/02/28/ 2008.

[43] C. R. Yonzon, D. A. Stuart, X. Zhang, A. D. McFarland, C. L. Haynes, and R. P. Van Duyne, "Towards advanced chemical and biological nanosensors-An overview," Talanta, vol. 67, no. 3, pp. 438$448,2005 / 09 / 15 / 2005$.

[44] P. Englebienne, "Use of colloidal gold surface plasmon resonance peak shift to infer affinity constants from the interactions between protein antigens and antibodies specific for single or multiple epitopes," Analyst, 10.1039/A804010l vol. 123, no. 7, pp. 1599-1603, 1998.

[45] K. M. Mayer et al., "A Label-Free Immunoassay Based Upon Localized Surface Plasmon Resonance of Gold Nanorods," ACS Nano, vol. 2, no. 4, pp. 687-692, 2008/04/01 2008.

[46] S. Lee, K. M. Mayer, and J. H. Hafner, "Improved Localized Surface Plasmon Resonance Immunoassay with Gold Bipyramid Substrates," Analytical Chemistry, vol. 81, no. 11, pp. 44504455, 2009/06/01 2009.

[47] A. J. Haes, L. Chang, W. L. Klein, and R. P. Van Duyne, "Detection of a Biomarker for Alzheimer's Disease from Synthetic and Clinical Samples Using a Nanoscale Optical Biosensor," Journal of the American Chemical Society, vol. 127, no. 7, pp. 2264-2271, 2005/02/01 2005.

[48] J. Wang, S. V. Boriskina, H. Wang, and B. M. Reinhard, "Illuminating Epidermal Growth Factor Receptor Densities on Filopodia through Plasmon Coupling," ACS nano, vol. 5, no. 8, pp. 6619$6628,07 / 222011$.

[49] A. Ahmed, "Enhancing Data Extraction from Localized Surface Plasmon Resonance Biosensors," PhD, ECE, McGill University, Canada, 2017.

[50] M. Liu, J. Chao, S. Deng, K. Wang, K. Li, and C. Fan, "Dark-field microscopy in imaging of plasmon resonant nanoparticles," Colloids and Surfaces B: Biointerfaces, vol. 124, pp. 111-117, 2014/12/01/ 2014.

[51] P. Singh, "LSPR Biosensing: Recent Advances and Approaches," in Reviews in Plasmonics 2016, C. D. Geddes, Ed. Cham: Springer International Publishing, 2017, pp. 211-238.

[52] T. D. Visser, "Surface plasmons at work?," Nature Physics, vol. 2, p. 509, 08/01/online 2006.

[53] T. W. Ebbesen, H. J. Lezec, H. F. Ghaemi, T. Thio, and P. A. Wolff, "Extraordinary optical transmission through sub-wavelength hole arrays," Nature, vol. 391, p. 667, 02/12/online 1998.

[54] C. Genet and T. W. Ebbesen, "Light in tiny holes," Nature, Review Article vol. 445, p. 39, 01/04/online 2007.

[55] M. Schwind, B. Kasemo, and I. Zorić, "Localized and Propagating Plasmons in Metal Films with Nanoholes," Nano Letters, vol. 13, no. 4, pp. 1743-1750, 2013/04/10 2013.

[56] S. F. Romanuik et al., "Detecting Antibodies Secreted by Trapped Cells Using Extraordinary Optical Transmission," IEEE Sensors Journal, vol. 11, no. 11, pp. 2732-2739, 2011. 
[57] M. Das, D. Hohertz, R. Nirwan, A. G. Brolo, K. L. Kavanagh, and R. Gordon, "Improved Performance of Nanohole Surface Plasmon Resonance Sensors by the Integrated Response Method," IEEE Photonics Journal, vol. 3, no. 3, pp. 441-449, 2011.

[58] A. A. Yanik, M. Huang, A. Artar, T. Y. Chang, and H. Altug, "Novel plasmonic biosensors molding the flow of light and fluidics at subdiffraction limit," in 2010 23rd Annual Meeting of the IEEE Photonics Society, 2010, pp. 162-163.

[59] S. Lal, S. Link, and N. J. Halas, "Nano-optics from sensing to waveguiding," Nature Photonics, Review Article vol. 1, p. 641, 11/01/online 2007.

[60] J. Dostálek, H. Vaisocherová, and J. Homola, "Multichannel surface plasmon resonance biosensor with wavelength division multiplexing," Sensors and Actuators B: Chemical, vol. 108, no. 1, pp. 758-764, 2005/07/22/ 2005.

[61] B. Nikoobakht and M. A. El-Sayed, "Preparation and Growth Mechanism of Gold Nanorods (NRs) Using Seed-Mediated Growth Method," Chemistry of Materials, vol. 15, no. 10, pp. 1957-1962, 2003/05/01 2003.

[62] S. Y. Yoo, D.-K. Kim, T. J. Park, E. K. Kim, E. Tamiya, and S. Y. Lee, "Detection of the Most Common Corneal Dystrophies Caused by BIGH3 Gene Point Mutations Using a Multispot Gold-Capped Nanoparticle Array Chip," Analytical Chemistry, vol. 82, no. 4, pp. 1349-1357, 2010/02/15 2010.

[63] S. Morokoshi, K. Ohhori, K. Mizukami, and H. Kitano, "Sensing Capabilities of Colloidal Gold Modified with a Self-Assembled Monolayer of a Glucose-Carrying Polymer Chain on a Glass Substrate," Langmuir, vol. 20, no. 20, pp. 8897-8902, 2004/09/01 2004.

[64] A. J. Haes, S. Zou, J. Zhao, G. C. Schatz, and R. P. Van Duyne, "Localized Surface Plasmon Resonance Spectroscopy near Molecular Resonances," Journal of the American Chemical Society, vol. 128, no. 33, pp. 10905-10914, 2006/08/01 2006.

[65] J. Zhao, A. Das, G. C. Schatz, S. G. Sligar, and R. P. Van Duyne, "Resonance Localized Surface Plasmon Spectroscopy: Sensing Substrate and Inhibitor Binding to Cytochrome P450," The Journal of Physical Chemistry C, vol. 112, no. 34, pp. 13084-13088, 2008/08/01 2008.

[66] A. Das, J. Zhao, G. C. Schatz, S. G. Sligar, and R. P. Van Duyne, "Screening of Type I and II Drug Binding to Human Cytochrome P450-3A4 in Nanodiscs by Localized Surface Plasmon Resonance Spectroscopy," Analytical Chemistry, vol. 81, no. 10, pp. 3754-3759, 2009/05/15 2009.

[67] W. J. Galush, S. A. Shelby, M. J. Mulvihill, A. Tao, P. Yang, and J. T. Groves, "A Nanocube Plasmonic Sensor for Molecular Binding on Membrane Surfaces," Nano Letters, vol. 9, no. 5, pp. 2077-2082, 2009/05/13 2009.

[68] T.-J. Lin, K.-T. Huang, and C.-Y. Liu, "Determination of organophosphorous pesticides by a novel biosensor based on localized surface plasmon resonance," Biosensors and Bioelectronics, vol. 22, no. 4, pp. 513-518, 2006/10/15/ 2006.

[69] C. Li, C. Wu, J. Zheng, J. Lai, C. Zhang, and Y. Zhao, "LSPR Sensing of Molecular Biothiols Based on Noncoupled Gold Nanorods," Langmuir, vol. 26, no. 11, pp. 9130-9135, 2010/06/01 2010.

[70] P. K. Jain, K. S. Lee, I. H. El-Sayed, and M. A. El-Sayed, "Calculated Absorption and Scattering Properties of Gold Nanoparticles of Different Size, Shape, and Composition: Applications in Biological Imaging and Biomedicine," The Journal of Physical Chemistry B, vol. 110, no. 14, pp. 7238-7248, 2006/04/01 2006.

[71] S. Link and M. A. El-Sayed, "Size and Temperature Dependence of the Plasmon Absorption of Colloidal Gold Nanoparticles," The Journal of Physical Chemistry B, vol. 103, no. 21, pp. 4212-4217, 1999/05/01 1999.

[72] H. Chen, X. Kou, Z. Yang, W. Ni, and J. Wang, "Shape- and Size-Dependent Refractive Index Sensitivity of Gold Nanoparticles," Langmuir, vol. 24, no. 10, pp. 5233-5237, 2008/05/01 2008.

[73] J. J. Mock, D. R. Smith, and S. Schultz, "Local Refractive Index Dependence of Plasmon Resonance Spectra from Individual Nanoparticles," Nano Letters, vol. 3, no. 4, pp. 485-491, 2003/04/01 2003. 
[74] Y. Sun and Y. Xia, "Increased Sensitivity of Surface Plasmon Resonance of Gold Nanoshells Compared to That of Gold Solid Colloids in Response to Environmental Changes," Analytical Chemistry, vol. 74, no. 20, pp. 5297-5305, 2002/10/01 2002.

[75] M. M. Miller and A. A. Lazarides, "Sensitivity of Metal Nanoparticle Surface Plasmon Resonance to the Dielectric Environment," The Journal of Physical Chemistry B, vol. 109, no. 46, pp. 2155621565, 2005/11/01 2005.

[76] S. Link and M. A. El-Sayed, "Spectral Properties and Relaxation Dynamics of Surface Plasmon Electronic Oscillations in Gold and Silver Nanodots and Nanorods," The Journal of Physical Chemistry B, vol. 103, no. 40, pp. 8410-8426, 1999/10/01 1999.

[77] L. J. Sherry, S.-H. Chang, G. C. Schatz, R. P. Van Duyne, B. J. Wiley, and Y. Xia, "Localized Surface Plasmon Resonance Spectroscopy of Single Silver Nanocubes," Nano Letters, vol. 5, no. 10, pp. 2034-2038, 2005/10/01 2005.

[78] P. K. Jain and M. A. El-Sayed, "Noble Metal Nanoparticle Pairs: Effect of Medium for Enhanced Nanosensing," Nano Letters, vol. 8, no. 12, pp. 4347-4352, 2008/12/10 2008.

[79] G. Mie, "Beiträge zur Optik trüber Medien, speziell kolloidaler Metallösungen," Annalen der Physik, vol. 330, no. 3, pp. 377-445, 2006.

[80] J. Parsons, C. P. Burrows, J. R. Sambles, and W. L. Barnes, "A comparison of techniques used to simulate the scattering of electromagnetic radiation by metallic nanostructures," Journal of Modern Optics, vol. 57, no. 5, pp. 356-365, 2010/03/10 2010.

[81] P. Chylek, "Absorption and scattering of light by small particles. By C. F. Bohren and d. R. Huffman," (in eng), Appl Opt, vol. 25, no. 18, p. 3166, Sep 151986.

[82] L. J. E. Anderson, K. M. Mayer, R. D. Fraleigh, Y. Yang, S. Lee, and J. H. Hafner, "Quantitative Measurements of Individual Gold Nanoparticle Scattering Cross Sections," The Journal of Physical Chemistry C, vol. 114, no. 25, pp. 11127-11132, 2010/07/01 2010.

[83] T. R. Jensen, M. L. Duval, K. L. Kelly, A. A. Lazarides, G. C. Schatz, and R. P. Van Duyne, "Nanosphere Lithography: Effect of the External Dielectric Medium on the Surface Plasmon Resonance Spectrum of a Periodic Array of Silver Nanoparticles," The Journal of Physical Chemistry B, vol. 103, no. 45, pp. 9846-9853, 1999/11/01 1999.

[84] G. R., "Über die Form ultramikroskopischer Goldteilchen," Annalen der Physik, vol. 342, no. 5, pp. 881-900, 1912.

[85] P. K. Jain, S. Eustis, and M. A. El-Sayed, "Plasmon Coupling in Nanorod Assemblies: Optical Absorption, Discrete Dipole Approximation Simulation, and Exciton-Coupling Model," The Journal of Physical Chemistry B, vol. 110, no. 37, pp. 18243-18253, 2006/09/01 2006.

[86] S. Link and M. A. El-Sayed, "Simulation of the Optical Absorption Spectra of Gold Nanorods as a Function of Their Aspect Ratio and the Effect of the Medium Dielectric Constant," The Journal of Physical Chemistry B, vol. 109, no. 20, pp. 10531-10532, 2005/05/01 2005.

[87] S. Link, M. B. Mohamed, and M. A. El-Sayed, "Simulation of the Optical Absorption Spectra of Gold Nanorods as a Function of Their Aspect Ratio and the Effect of the Medium Dielectric Constant," The Journal of Physical Chemistry B, vol. 103, no. 16, pp. 3073-3077, 1999/04/01 1999.

[88] C. L. Nehl and J. H. Hafner, "Shape-dependent plasmon resonances of gold nanoparticles," Journal of Materials Chemistry, 10.1039/B714950F vol. 18, no. 21, pp. 2415-2419, 2008.

[89] S. Zeng, D. Baillargeat, H.-P. Ho, and K.-T. Yong, "Nanomaterials enhanced surface plasmon resonance for biological and chemical sensing applications," Chemical Society Reviews, 10.1039/C3CS60479A vol. 43, no. 10, pp. 3426-3452, 2014.

[90] S. Schultz, D. R. Smith, J. J. Mock, and D. A. Schultz, "Single-target molecule detection with nonbleaching multicolor optical immunolabels," (in eng), Proc Natl Acad Sci U S A, vol. 97, no. 3, pp. 996-1001, Feb 12000. 
[91] J. N. Anker, W. P. Hall, O. Lyandres, N. C. Shah, J. Zhao, and R. P. Van Duyne, "Biosensing with plasmonic nanosensors," Nature Materials, Review Article vol. 7, p. 442, 06/01/online 2008.

[92] S. Peiris, J. McMurtrie, and H. Zhu, Metal nanoparticle photocatalysts: Emerging processes for green organic synthesis. 2015.

[93] T. R. Jensen, M. D. Malinsky, C. L. Haynes, and R. P. Van Duyne, "Nanosphere Lithography: Tunable Localized Surface Plasmon Resonance Spectra of Silver Nanoparticles," The Journal of Physical Chemistry B, vol. 104, no. 45, pp. 10549-10556, 2000/11/01 2000.

[94] C. R. Yonzon, D. A. Stuart, X. Zhang, A. D. McFarland, C. L. Haynes, and R. P. Van Duyne, "Towards advanced chemical and biological nanosensors-An overview," (in eng), Talanta, vol. 67, no. 3, pp. 438-48, Sep 152005.

[95] K. A. Willets and R. P. Van Duyne, "Localized surface plasmon resonance spectroscopy and sensing," (in English), Annual Review of Physical Chemistry, vol. 58, pp. 267-297, 2007.

[96] A. B. Dahlin, J. O. Tegenfeldt, and F. Höök, "Improving the Instrumental Resolution of Sensors Based on Localized Surface Plasmon Resonance," Analytical Chemistry, vol. 78, no. 13, pp. 44164423, 2006/07/01 2006.

[97] D. A. Stuart, A. J. Haes, A. D. McFarland, S. Nie, and R. P. V. Duyne, "Refractive-index-sensitive, plasmon-resonant-scattering, and surface-enhanced Raman-scattering nanoparticles and arrays as biological sensing platforms," in Biomedical Optics 2004, 2004, vol. 5327, p. 14: SPIE.

[98] A. Unger, U. Rietzler, R. Berger, and M. Kreiter, "Sensitivity of Crescent-Shaped Metal Nanoparticles to Attachment of Dielectric Colloids," Nano Letters, vol. 9, no. 6, pp. 2311-2315, 2009/06/10 2009.

[99] A. J. Haes, S. Zou, G. C. Schatz, and R. P. Van Duyne, "A Nanoscale Optical Biosensor: The Long Range Distance Dependence of the Localized Surface Plasmon Resonance of Noble Metal Nanoparticles," The Journal of Physical Chemistry B, vol. 108, no. 1, pp. 109-116, 2004/01/01 2004.

[100] A. J. Haes, S. Zou, G. C. Schatz, and R. P. Van Duyne, "Nanoscale Optical Biosensor: Short Range Distance Dependence of the Localized Surface Plasmon Resonance of Noble Metal Nanoparticles," The Journal of Physical Chemistry B, vol. 108, no. 22, pp. 6961-6968, 2004/06/01 2004.

[101] M. D. Malinsky, K. L. Kelly, G. C. Schatz, and R. P. Van Duyne, "Chain Length Dependence and Sensing Capabilities of the Localized Surface Plasmon Resonance of Silver Nanoparticles Chemically Modified with Alkanethiol Self-Assembled Monolayers," Journal of the American Chemical Society, vol. 123, no. 7, pp. 1471-1482, 2001/02/01 2001.

[102] G. J. Nusz, A. C. Curry, S. M. Marinakos, A. Wax, and A. Chilkoti, "Rational Selection of Gold Nanorod Geometry for Label-Free Plasmonic Biosensors," ACS Nano, vol. 3, no. 4, pp. 795-806, 2009/04/28 2009.

[103] J. Becker, A. Trügler, A. Jakab, U. Hohenester, and C. Sönnichsen, "The Optimal Aspect Ratio of Gold Nanorods for Plasmonic Bio-sensing," Plasmonics, vol. 5, no. 2, pp. 161-167, 2010/06/01 2010.

[104] J. Aaron et al., "Plasmon resonance coupling of metal nanoparticles for molecular imaging of carcinogenesis in vivo," (in eng), J Biomed Opt, vol. 12, no. 3, p. 034007, May-Jun 2007.

[105] D. Lasne et al., "Single Nanoparticle Photothermal Tracking (SNaPT) of 5-nm Gold Beads in Live Cells," Biophysical Journal, vol. 91, no. 12, pp. 4598-4604, 09/22 05/22/received 07/21/accepted 2006.

[106] K. J. Lee, P. D. Nallathamby, L. M. Browning, C. J. Osgood, and X. H. Xu, "In vivo imaging of transport and biocompatibility of single silver nanoparticles in early development of zebrafish embryos," (in eng), ACS Nano, vol. 1, no. 2, pp. 133-43, Sep 2007. 
[107] D. M. SHOTTON, "Confocal scanning optical microscopy and its applications for biological specimens," Journal of Cell Science, vol. 94, no. 2, pp. 175-206, 1989.

[108] X. H. Xu, W. J. Brownlow, S. V. Kyriacou, Q. Wan, and J. J. Viola, "Real-time probing of membrane transport in living microbial cells using single nanoparticle optics and living cell imaging," (in eng), Biochemistry, vol. 43, no. 32, pp. 10400-13, Aug 172004.

[109] X.-H. N. Xu, J. Chen, R. B. Jeffers, and S. Kyriacou, "Direct Measurement of Sizes and Dynamics of Single Living Membrane Transporters Using Nanooptics," Nano Letters, vol. 2, no. 3, pp. 175-182, 2002/03/01 2002.

[110] A. D. McFarland and R. P. Van Duyne, "Single Silver Nanoparticles as Real-Time Optical Sensors with Zeptomole Sensitivity," Nano Letters, vol. 3, no. 8, pp. 1057-1062, 2003/08/01 2003.

[111] A. V. Whitney et al., "Localized Surface Plasmon Resonance Nanosensor: A High-Resolution Distance-Dependence Study Using Atomic Layer Deposition," The Journal of Physical Chemistry B, vol. 109, no. 43, pp. 20522-20528, 2005/11/01 2005.

[112] J. Zhao, X. Zhang, C. R. Yonzon, A. J. Haes, and R. P. Van Duyne, "Localized surface plasmon resonance biosensors," Nanomedicine, vol. 1, no. 2, pp. 219-228, 2006/08/01 2006.

[113] B. M. Reinhard, S. Sheikholeslami, A. Mastroianni, A. P. Alivisatos, and J. Liphardt, "Use of plasmon coupling to reveal the dynamics of DNA bending and cleavage by single EcoRV restriction enzymes," Proceedings of the National Academy of Sciences, 10.1073/pnas.0607826104 vol. 104, no. 8, p. 2667, 2007.

[114] C. Sönnichsen, B. M. Reinhard, J. Liphardt, and A. P. Alivisatos, "A molecular ruler based on plasmon coupling of single gold and silver nanoparticles," Nature Biotechnology, vol. 23, p. 741, 05/22/online 2005.

[115] J. Zhao, L. Jensen, J. Sung, S. Zou, G. C. Schatz, and R. P. Van Duyne, "Interaction of Plasmon and Molecular Resonances for Rhodamine 6G Adsorbed on Silver Nanoparticles," Journal of the American Chemical Society, vol. 129, no. 24, pp. 7647-7656, 2007/06/01 2007.

[116] R. Elghanian, J. J. Storhoff, R. C. Mucic, R. L. Letsinger, and C. A. Mirkin, "Selective Colorimetric Detection of Polynucleotides Based on the Distance-Dependent Optical Properties of Gold Nanoparticles," Science, 10.1126/science.277.5329.1078 vol. 277, no. 5329, p. 1078, 1997.

[117] F. Taleb and H. Fromm, Calcium/calmodulin-binding transcription activators in plants and animals. 2004, pp. 297-315.

[118] D. E. Ingber, "Cellular mechanotransduction: putting all the pieces together again," The FASEB Journal, vol. 20, no. 7, pp. 811-827, 2006/05/01 2006.

[119] P. K. Jain, I. H. El-Sayed, and M. A. El-Sayed, "Au nanoparticles target cancer," Nano Today, vol. 2, no. 1, pp. 18-29, 2007/02/01/ 2007.

[120] W. Rechberger, A. Hohenau, A. Leitner, J. R. Krenn, B. Lamprecht, and F. R. Aussenegg, "Optical properties of two interacting gold nanoparticles," Optics Communications, vol. 220, no. 1, pp. 137141, 2003/05/01/ 2003.

[121] B. M. Reinhard, M. Siu, H. Agarwal, A. P. Alivisatos, and J. Liphardt, "Calibration of Dynamic Molecular Rulers Based on Plasmon Coupling between Gold Nanoparticles," Nano Letters, vol. 5, no. 11, pp. 2246-2252, 2005/11/01 2005.

[122] J. Homola, "Present and future of surface plasmon resonance biosensors," Analytical and Bioanalytical Chemistry, journal article vol. 377, no. 3, pp. 528-539, October 012003.

[123] C. E. Jin et al., "Rapid and accurate detection of KRAS mutations in colorectal cancers using the isothermal-based optical sensor for companion diagnostics," Oncotarget, vol. 8, no. 48, pp. 8386083871, Oct 2017.

[124] K. H. Su, Q. H. Wei, X. Zhang, J. J. Mock, D. R. Smith, and S. Schultz, "Interparticle Coupling Effects on Plasmon Resonances of Nanogold Particles," Nano Letters, vol. 3, no. 8, pp. 1087-1090, 2003/08/01 2003. 
[125] P. J. R. Roche et al., "Real time plasmonic qPCR: how fast is ultra-fast? 30 cycles in 54 seconds," (in eng), Analyst, vol. 142, no. 10, pp. 1746-1755, May 212017.

[126] S. A. Maier and H. A. Atwater, "Plasmonics: Localization and guiding of electromagnetic energy in metal/dielectric structures," Journal of Applied Physics, vol. 98, no. 1, p. 011101, 2005.

[127] A. O. Govorov and H. H. Richardson, "Generating heat with metal nanoparticles," Nano Today, vol. 2, no. 1, pp. 30-38, 2007/02/01/ 2007.

[128] A. G. Russell, M. D. McKnight, J. A. Hestekin, and D. K. Roper, "Thermodynamics of Optoplasmonic Heating in Fluid-Filled Gold-Nanoparticle-Plated Capillaries," Langmuir, vol. 27, no. 12, pp. 77997805, 2011/06/21 2011.

[129] H. H. Richardson, M. T. Carlson, P. J. Tandler, P. Hernandez, and A. O. Govorov, "Experimental and Theoretical Studies of Light-to-Heat Conversion and Collective Heating Effects in Metal Nanoparticle Solutions," Nano Letters, vol. 9, no. 3, pp. 1139-1146, 2009/03/11 2009.

[130] P. J. Roche et al., "Demonstration of a plasmonic thermocycler for the amplification of human androgen receptor DNA," (in eng), Analyst, vol. 137, no. 19, pp. 4475-81, Oct 72012. 